Network Working Group

Request for Comments: 5060

Category: Standards Track
R. Sivaramu Cisco Systems

J. Lingard

Arastra, Inc

D. McWalter

Data Connection Ltd

B. Joshi

Infosys Technologies Ltd

A. Kessler

Cisco Systems

January 2008

\title{
Protocol Independent Multicast MIB
}

\section{Status of This Memo}

This document specifies an Internet standards track protocol for the Internet community, and requests discussion and suggestions for improvements. Please refer to the current edition of the "Internet Official Protocol Standards" (STD 1) for the standardization state and status of this protocol. Distribution of this memo is unlimited.

\section{Abstract}

This memo defines a portion of the Management Information Base (MIB) for use with network management protocols in the Internet community. In particular, it describes managed objects used for managing the Protocol Independent Multicast (PIM) protocols: PIM-SM (Sparse Mode), BIDIR-PIM (Bidirectional), and PIM-DM (Dense Mode). This document is part of work in progress to obsolete RFC 2934, and is to be preferred where the two documents overlap. This document does not obsolete RFC 2934 .

Table of Contents

1. Introduction . . . . . . . . . . . . . . . . . . . . . 2

2. Terminology . . . . . . . . . . . . . . . . . . . . . . . . 2

3. The Internet-Standard Management Framework . . . . . . . . . . 2

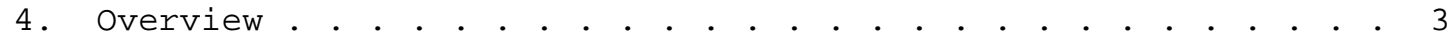

5. Definitions . . . . . . . . . . . . . . . . . . . . . 4

6. Security Considerations . . . . . . . . . . . . . . . . . 82

7. IANA Considerations . . . . . . . . . . . . . . . . . 86

8. Acknowledgements . . . . . . . . . . . . . . . . . . 86

9. References . . . . . . . . . . . . . . . . . . . . 86

9.1. Normative References . . . . . . . . . . . . . . 86

9.2. Informative References . . . . . . . . . . . . . . . 87 


\section{Introduction}

This memo defines a portion of the Management Information Base (MIB) for use with network management protocols in the Internet community. In particular, it describes managed objects used for managing the Protocol Independent Multicast (PIM) protocols (PIM-SM [RFC4601], BIDIR-PIM [RFC5015], and PIM-DM [RFC3973]).

This document is part of work in progress to obsolete RFC 2934 [RFC2934]. RFC 2934 defined an experimental MIB module for managing the PIM protocols. The MIB module defined by this document is a reworking of the MIB module from RFC 2934, with major changes that include the following.

- This MIB module is independent of IP version, whereas RFC 2934 only supported IPv4.

- This MIB module includes support for managing BIDIR-PIM.

- This MIB module retains limited support for managing PIM-DM [RFC3973], but that is no longer its primary purpose.

- This MIB module does not include support for managing PIM-SM V1.

- This MIB module does not depend on the IPv4 Multicast Routing MIB defined in RFC 2932 [RFC2932].

- This MIB module includes support for configuring static Rendezvous Points (RPS).

- This MIB module includes support for configuring anycast RPs [RFC4610].

2. Terminology

The key words "MUST", "MUST NOT", "REQUIRED", "SHALL", "SHALL NOT", "SHOULD", "SHOULD NOT", "RECOMMENDED", "MAY", and "OPTIONAL" in this document are to be interpreted as described in RFC 2119 [RFC2119].

3. The Internet-Standard Management Framework

For a detailed overview of the documents that describe the current Internet-Standard Management Framework, please refer to section 7 of RFC 3410 [RFC3410].

Managed objects are accessed via a virtual information store, termed the Management Information Base or MIB. MIB objects are generally accessed through the Simple Network Management Protocol (SNMP). 
Objects in the MIB are defined using the mechanisms defined in the Structure of Management Information (SMI). This memo specifies a MIB module that is compliant to the SMIV2, which is described in STD 58, RFC 2578 [RFC2578], STD 58, RFC 2579 [RFC2579] and STD 58, RFC 2580 [RFC2580].

4. Overview

This MIB module contains the following tables.

1. The PIM Interface Table, which contains one row per IP version for each interface of the router which is running PIM.

2. The PIM Neighbor Table, which contains one row for each of the router's PIM neighbors.

3. The PIM Neighbor Secondary Address Table, which contains one row for each secondary address advertised by each of the router's PIM neighbors.

4. The PIM (*,G) State Table, which contains one row for each group for which PIM has (*,G) state.

5. The PIM (*, G, I) State Table, which contains one row for each group and interface for which PIM has interface-specific (*,G) state.

6. The PIM (S,G) State Table, which contains one row for each source and group for which PIM has $(S, G)$ state.

7. The PIM (S, G, I) State Table, which contains one row for each source, group, and interface for which PIM has interfacespecific $(S, G)$ state.

8. The PIM (S, G, rpt) State Table, which contains one row for each source and group for which PIM has (S, G, rpt) state.

9. The PIM (S, G, rpt, I) State Table, which contains one row for each source, group, and interface for which PIM has interfacespecific (S, G, rpt) state.

10. The PIM Bidir DF-Election Table, which contains one row per interface for each Rendezvous Point (RP) for which Bidirectional-PIM Designated Forwarder (DF) election state is maintained. 
11. The PIM Static RP Table, which contains one row per range of multicast group addresses for which a particular configured RP should be used.

12. The PIM Group Mapping Table, which contains one row for each mapping from a multicast group address prefix to the PIM mode and RP address to use for groups within that group prefix, regardless of the source of the group mapping information.

13. The PIM Anycast-RP Set Table, which contains one row for each RP within each Anycast-RP set of which the local router is a member.

This MIB module uses textual conventions defined in the IF-MIB [RFC2863], the INET-ADDRESS-MIB [RFC4001], and the IANA-RTPROTO-MIB [RTPROTO]. This MIB module REFERENCEs [RFC3376], [RFC3569],

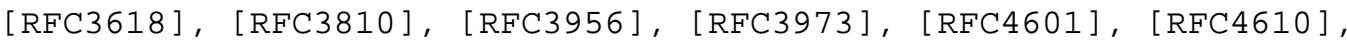

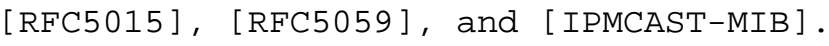

5. Definitions

PIM-STD-MIB DEFINITIONS : := BEGIN

IMPORTS

MODULE-IDENTITY, OBJECT-TYPE, mib-2, NOTIFICATION-TYPE, Unsigned32, Counter32, Counter64, Gauge32, TimeTicks FROM SNMPV2-SMI -- [RFC2578] TEXTUAL-CONVENTION, Rowstatus, Truthvalue, StorageType MODULE-COMPLIANCE, OBJECT-GROUP, NOTIFICATION-GROUP InterfaceIndexOrZero, InterfaceIndex InetAddress Type, InetAddressPrefixLength, InetAddress, InetVersion FROM INET-ADDRESS-MIB -- [RFC4001] IANAipRouteProtocol

pimStdMIB MODULE-IDENTITY

LAST-UPDATED "200711020000Z" -- 2 November 2007

ORGANIZATION

"IETF Protocol Independent Multicast (PIM) Working Group" CONTACT-INFO

"Email: pimeietf.org

WG charter: 


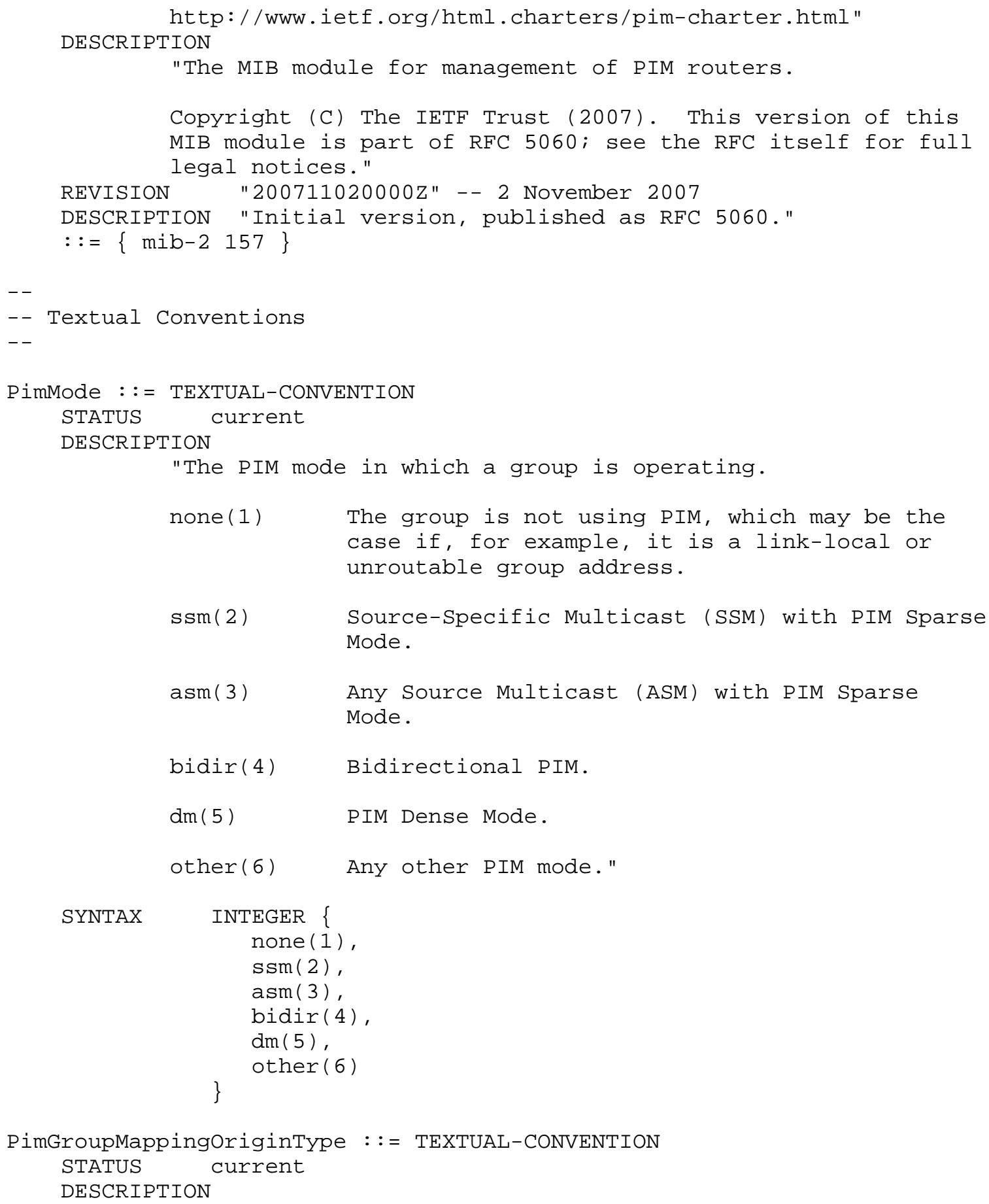




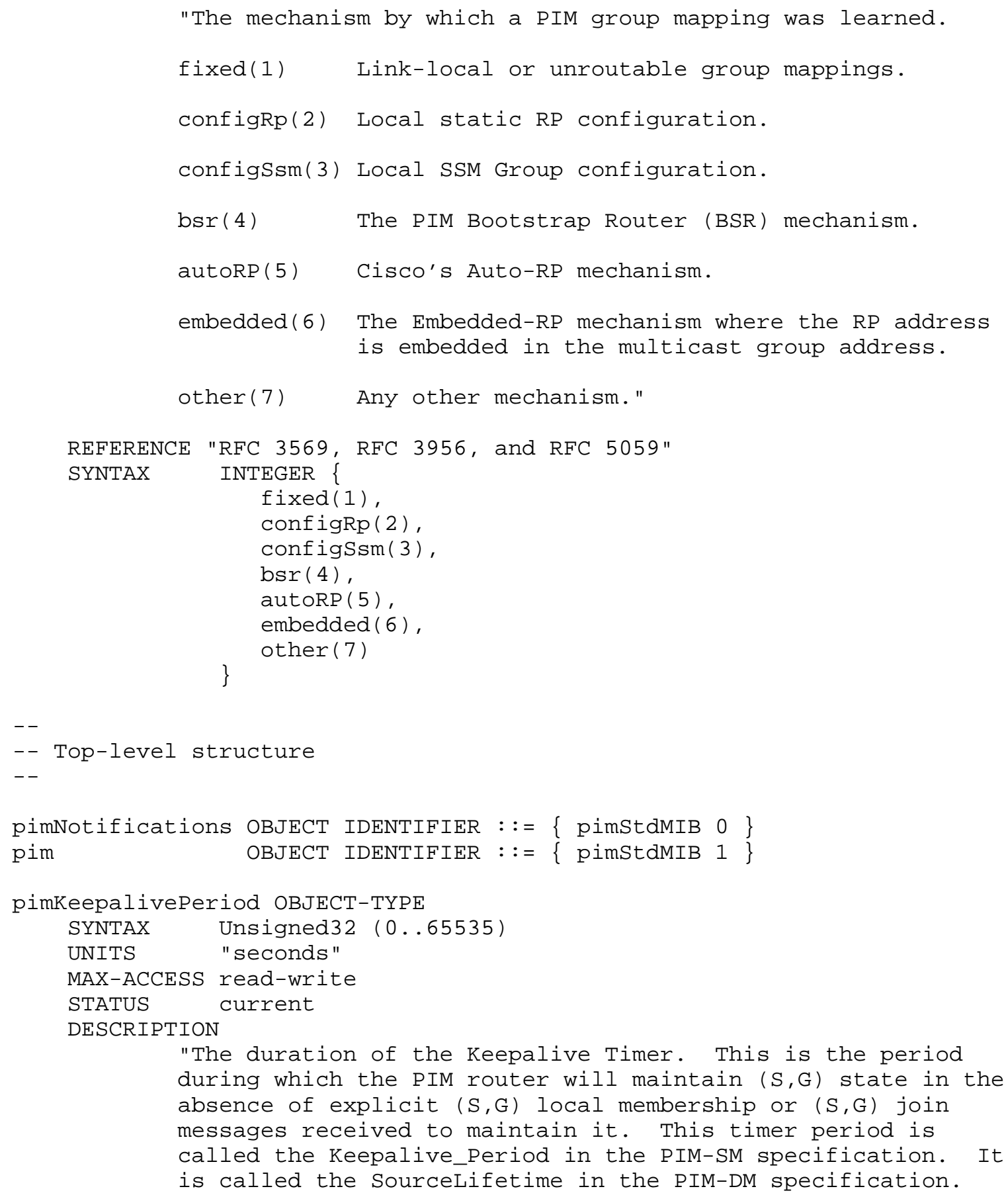




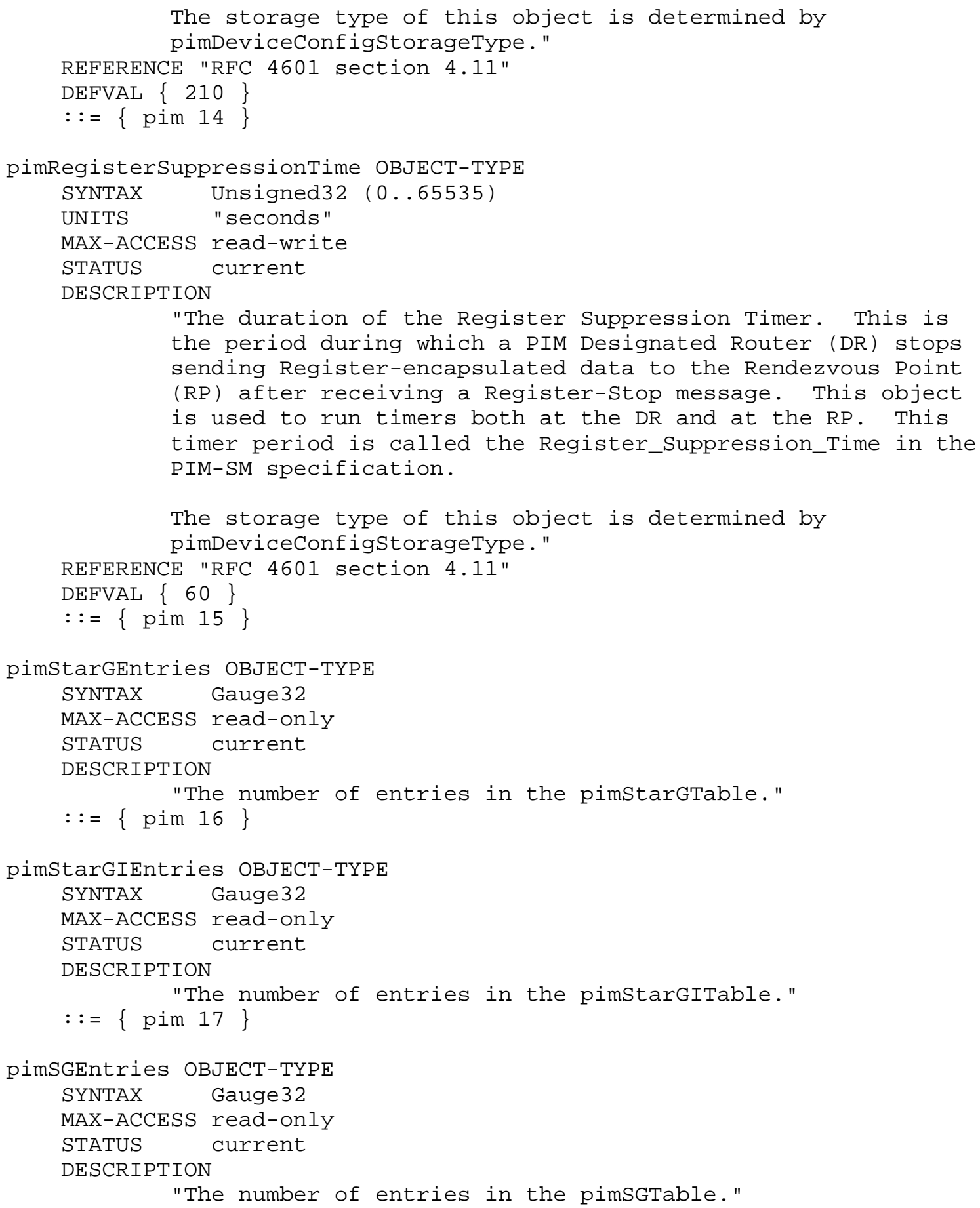




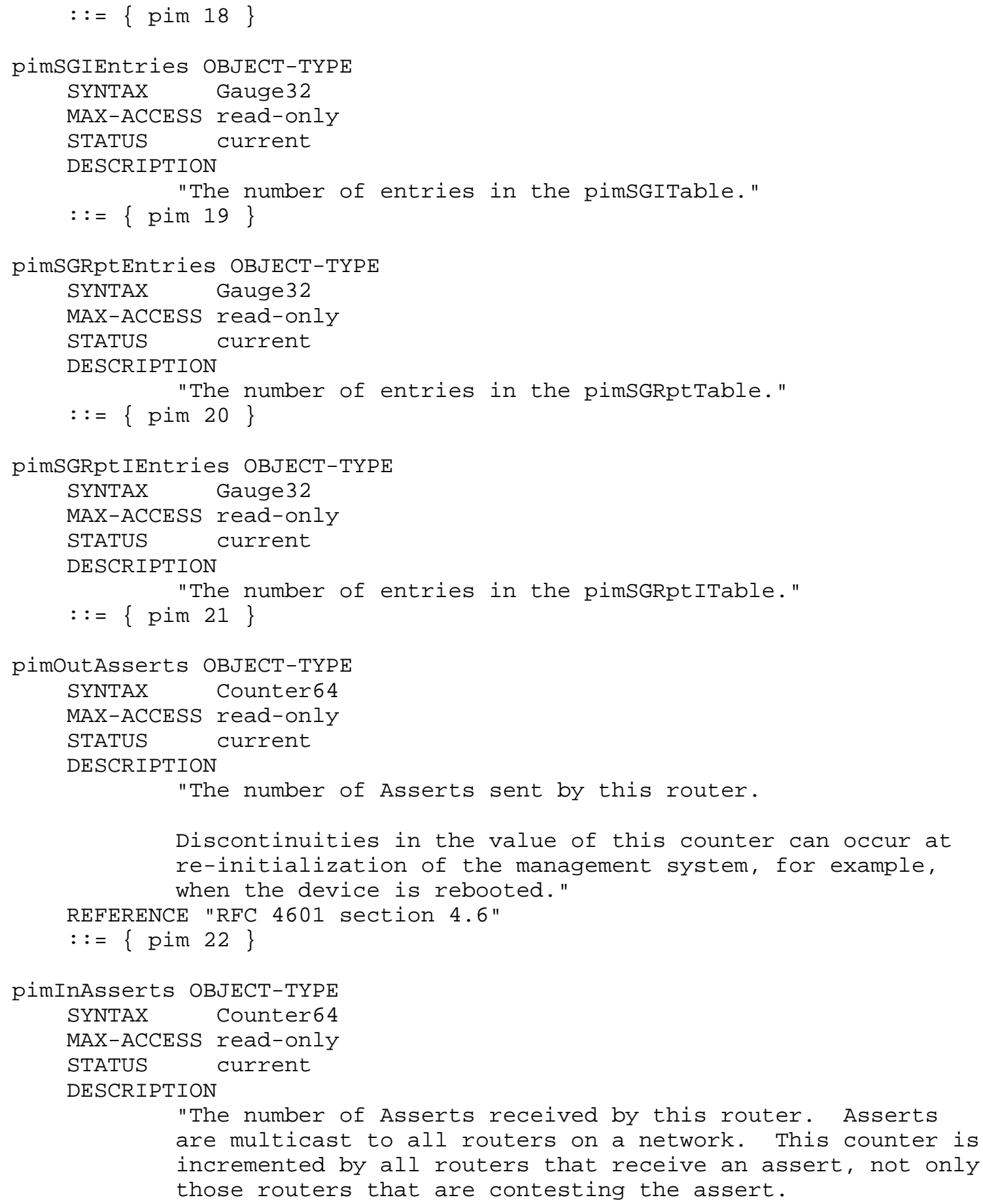




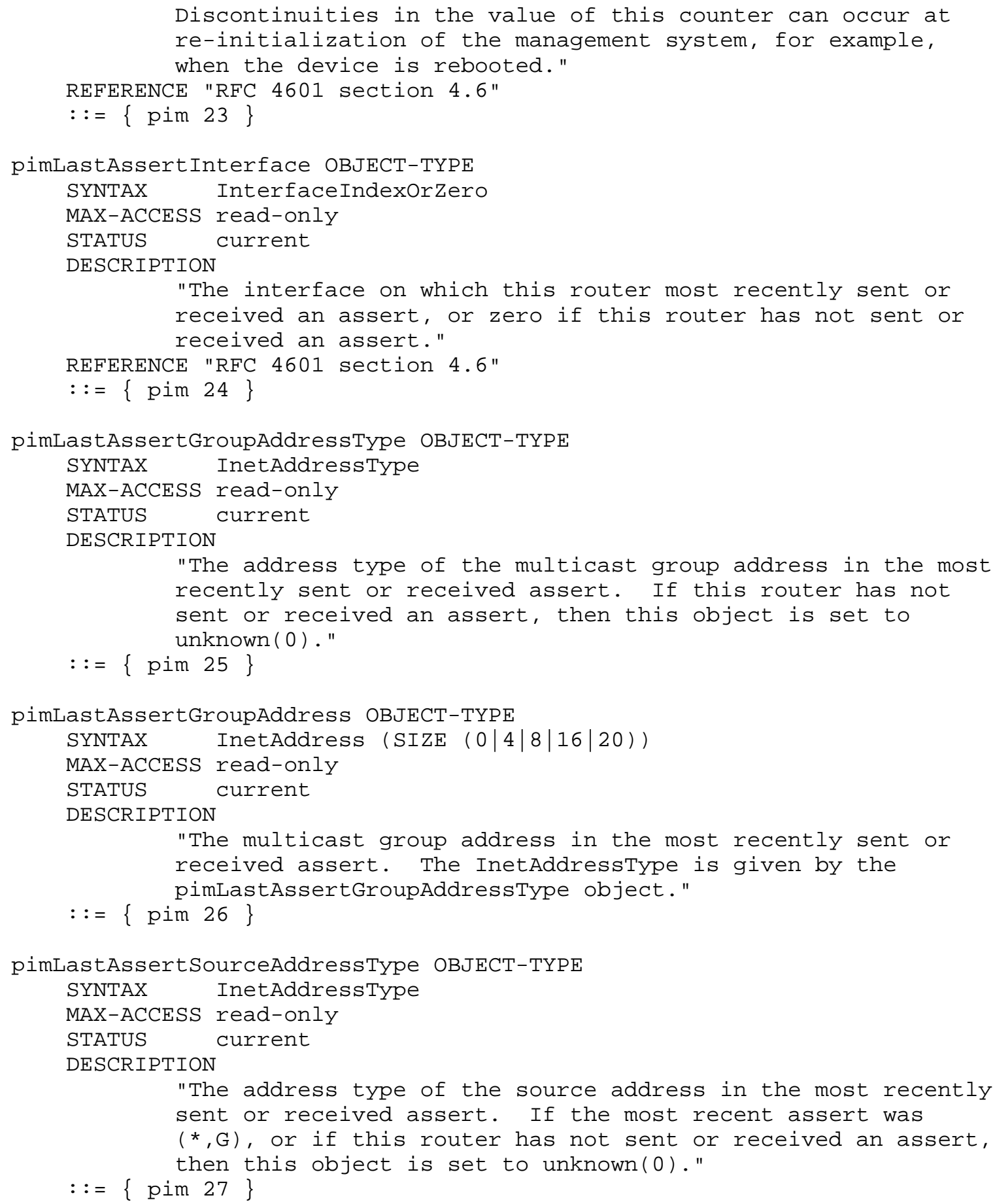




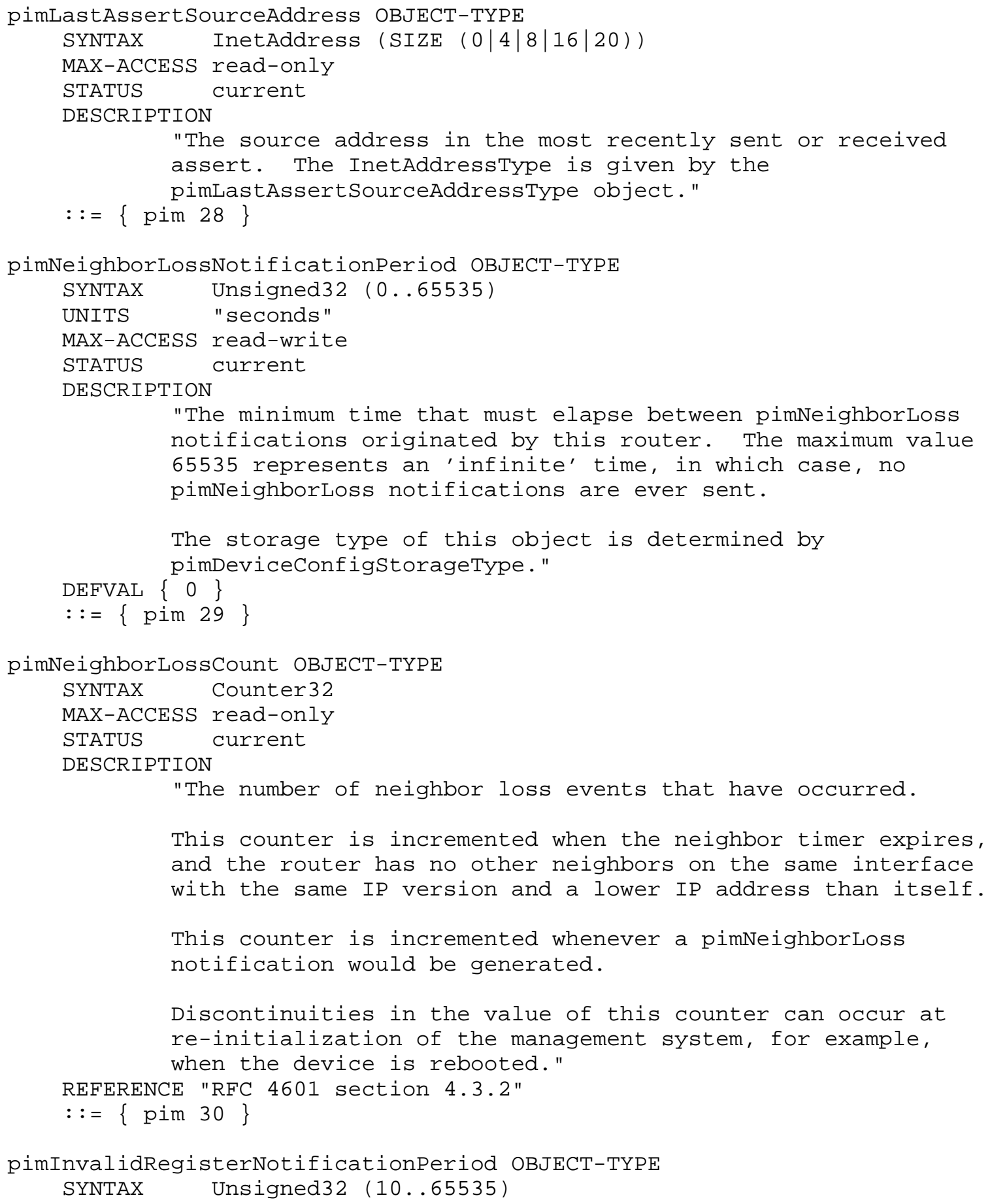




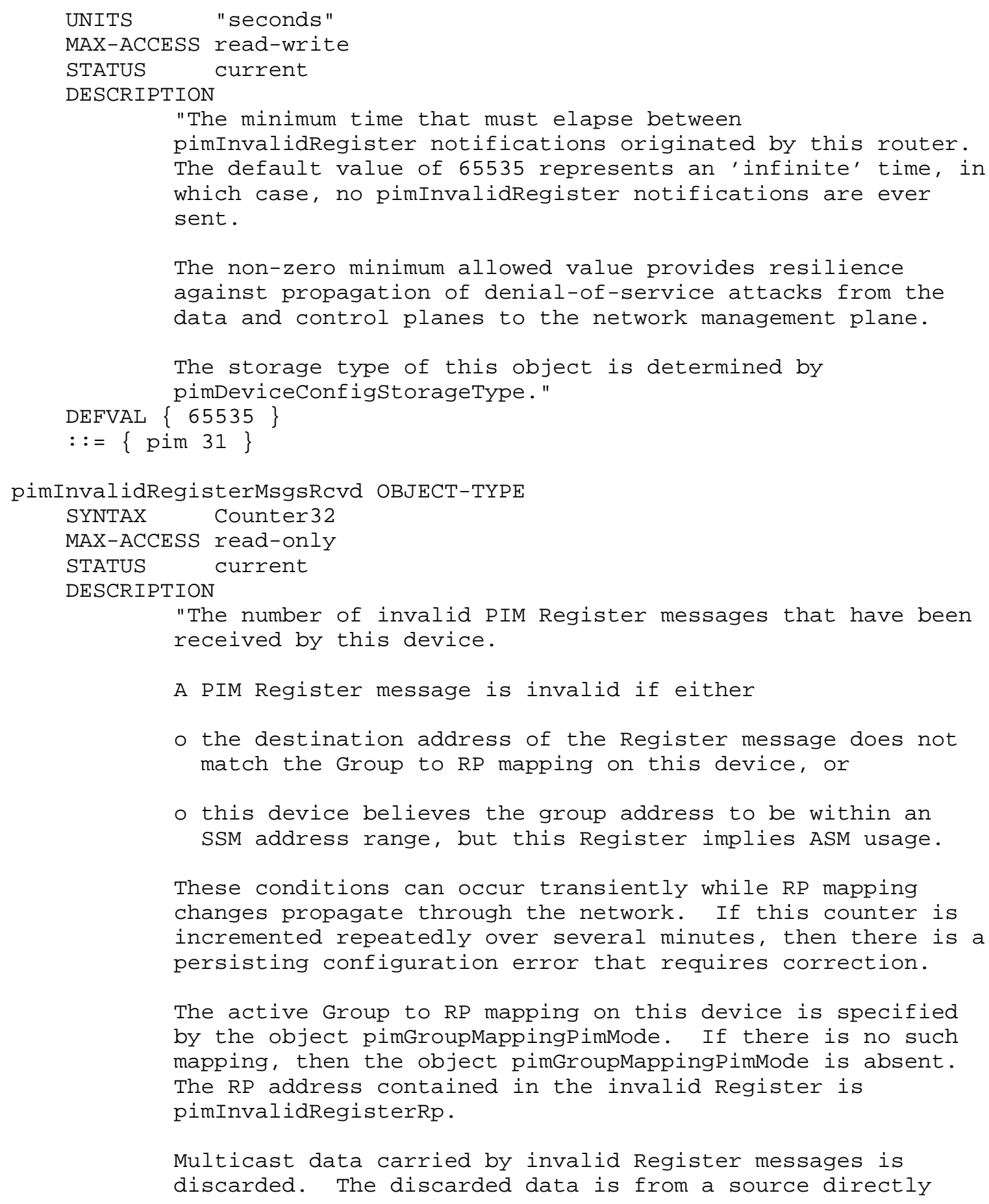




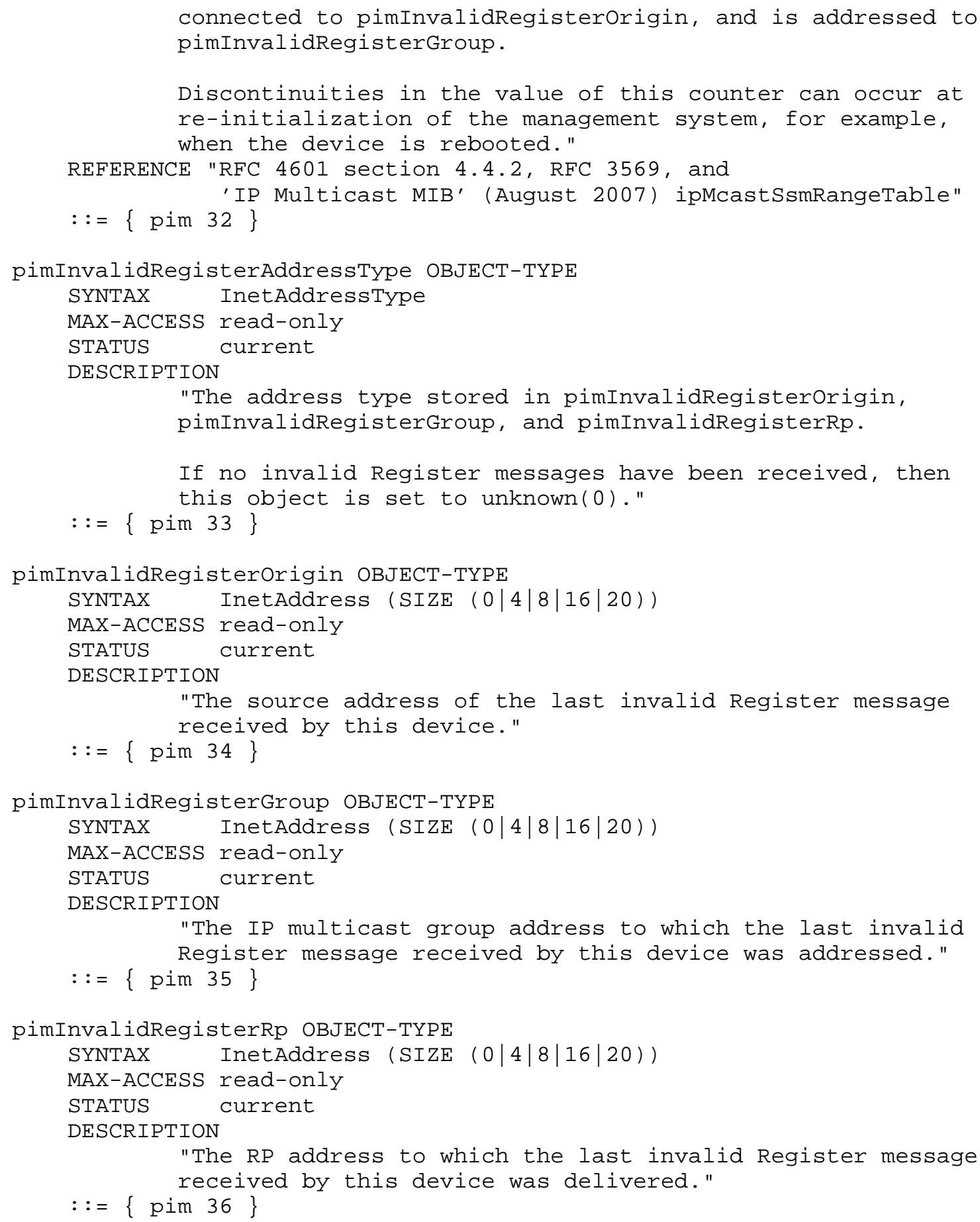




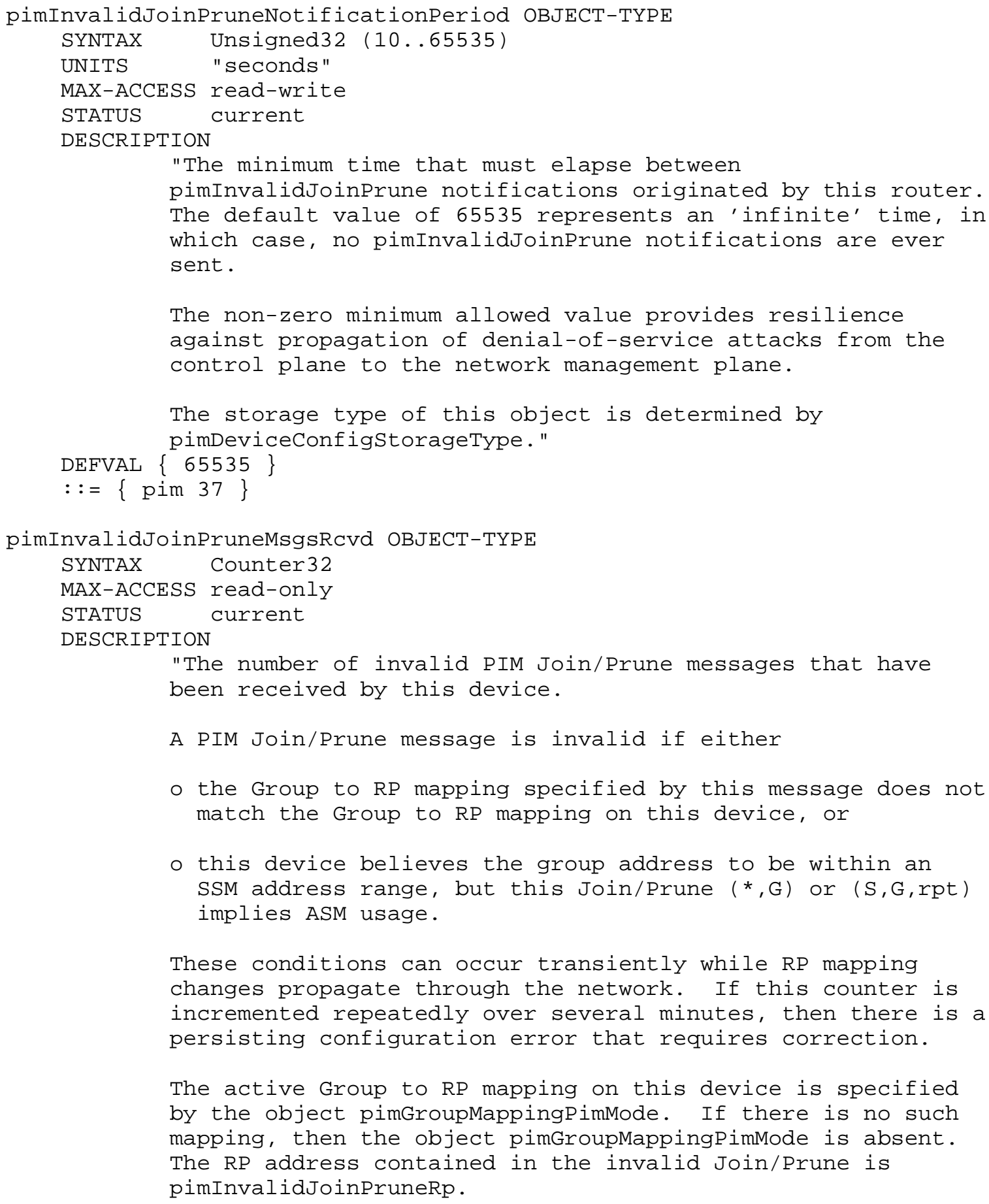




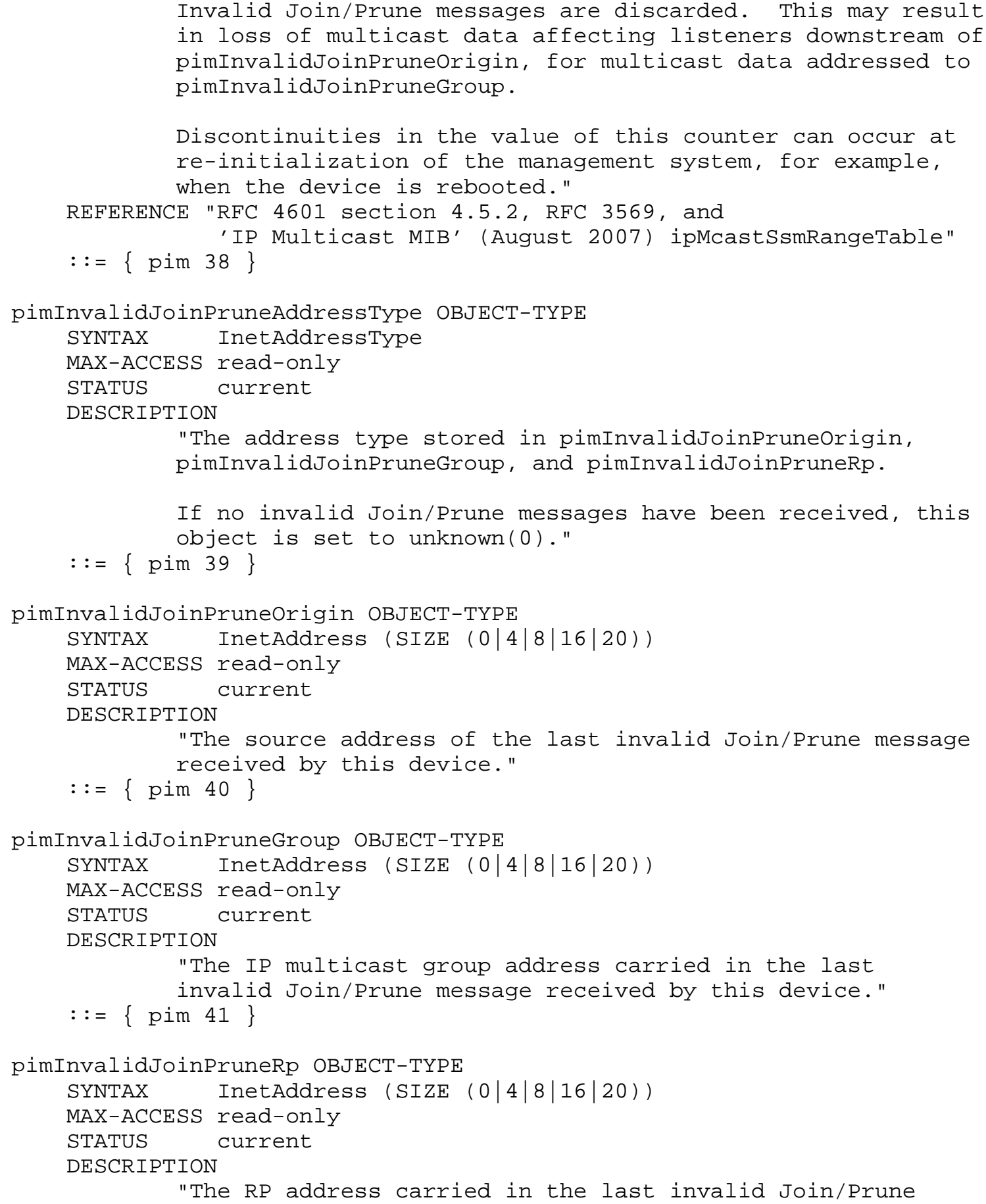




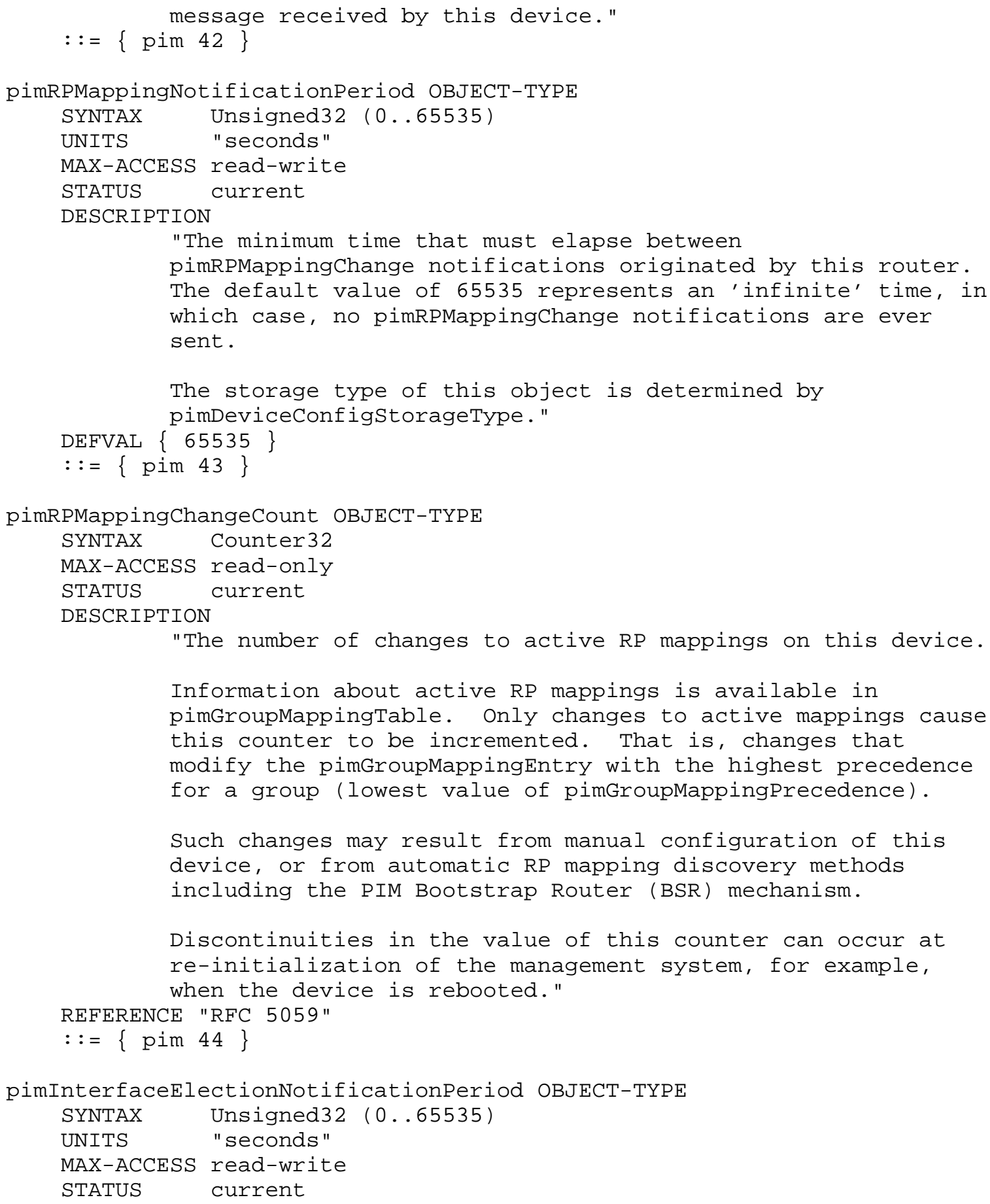




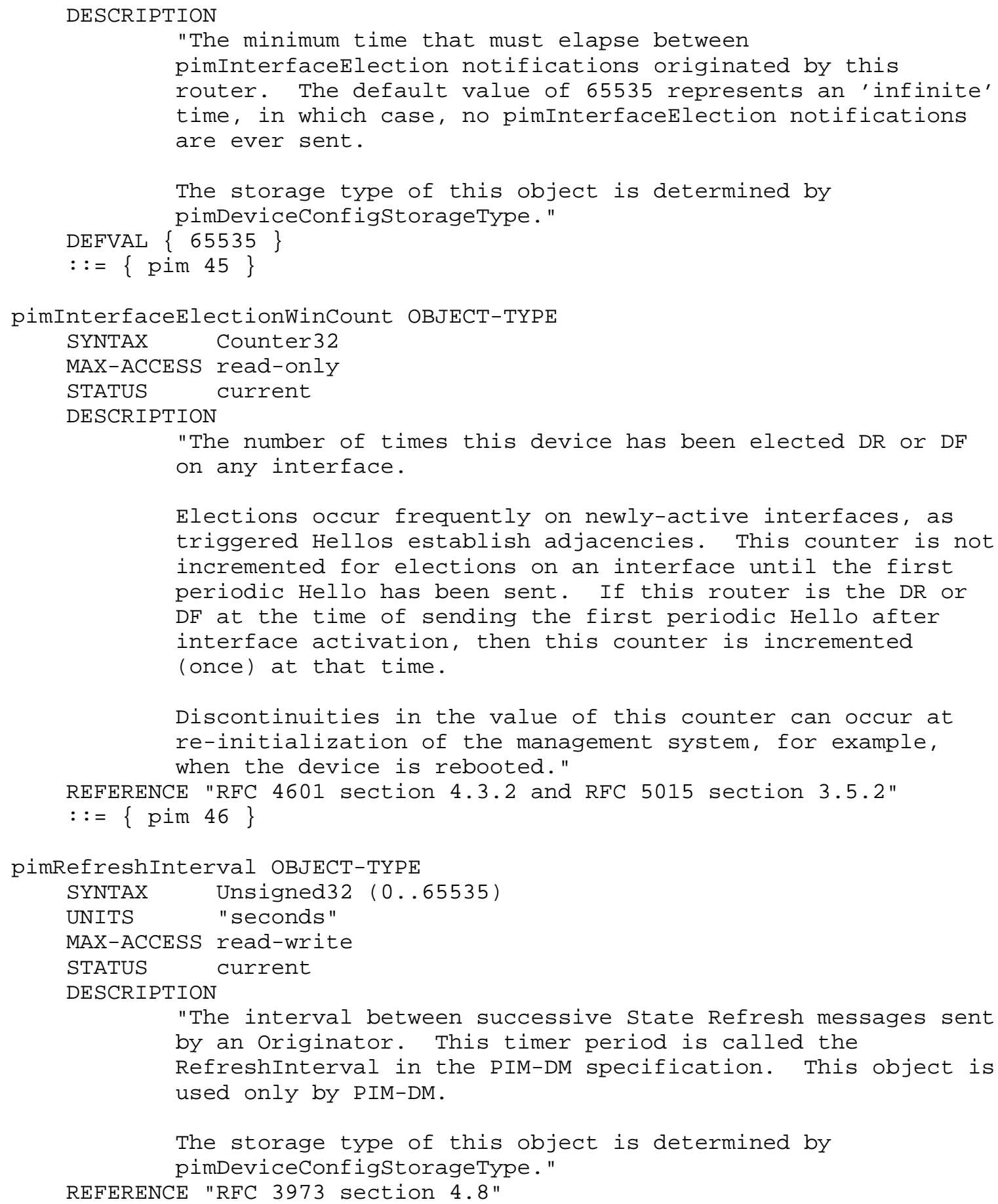




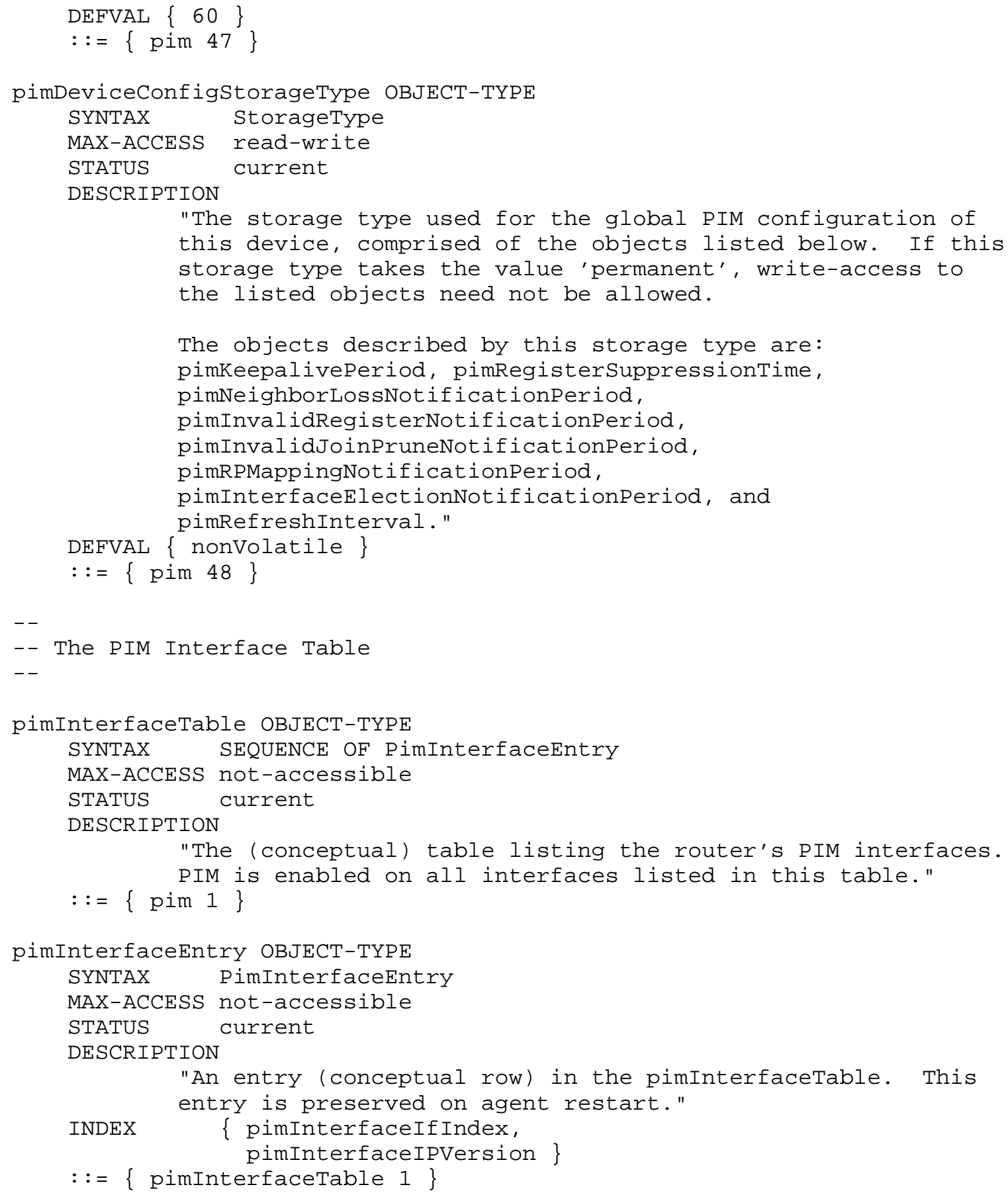




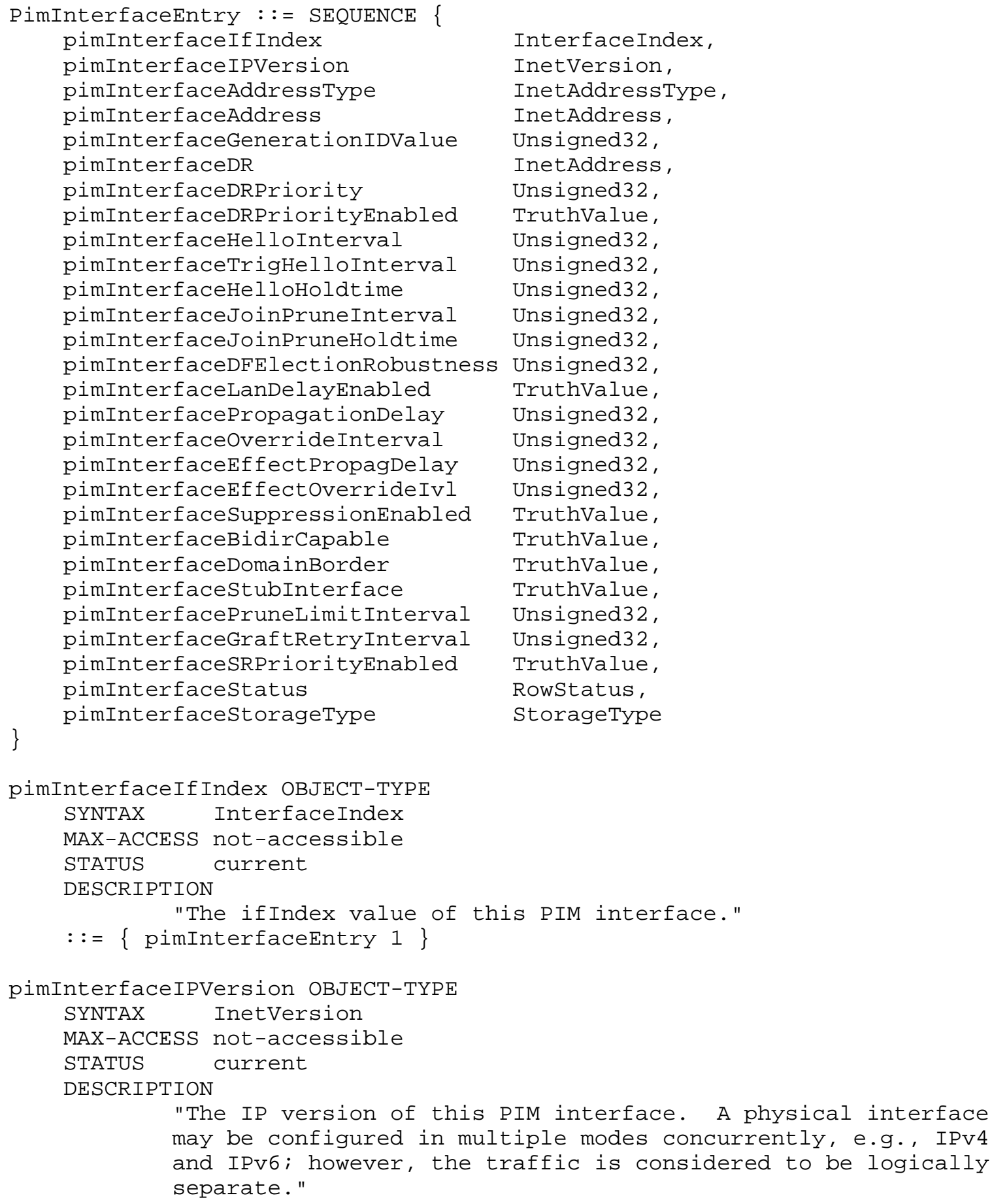




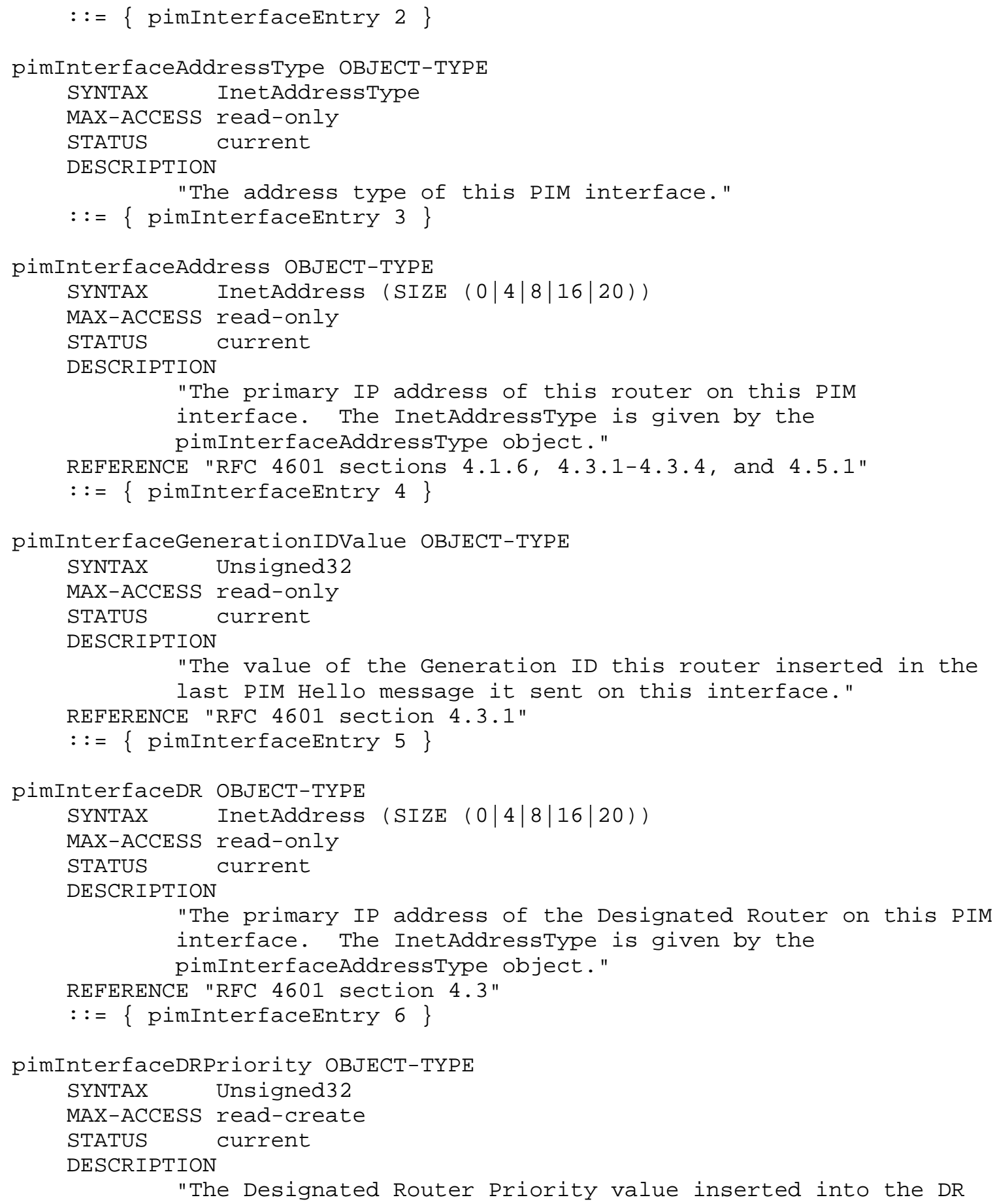




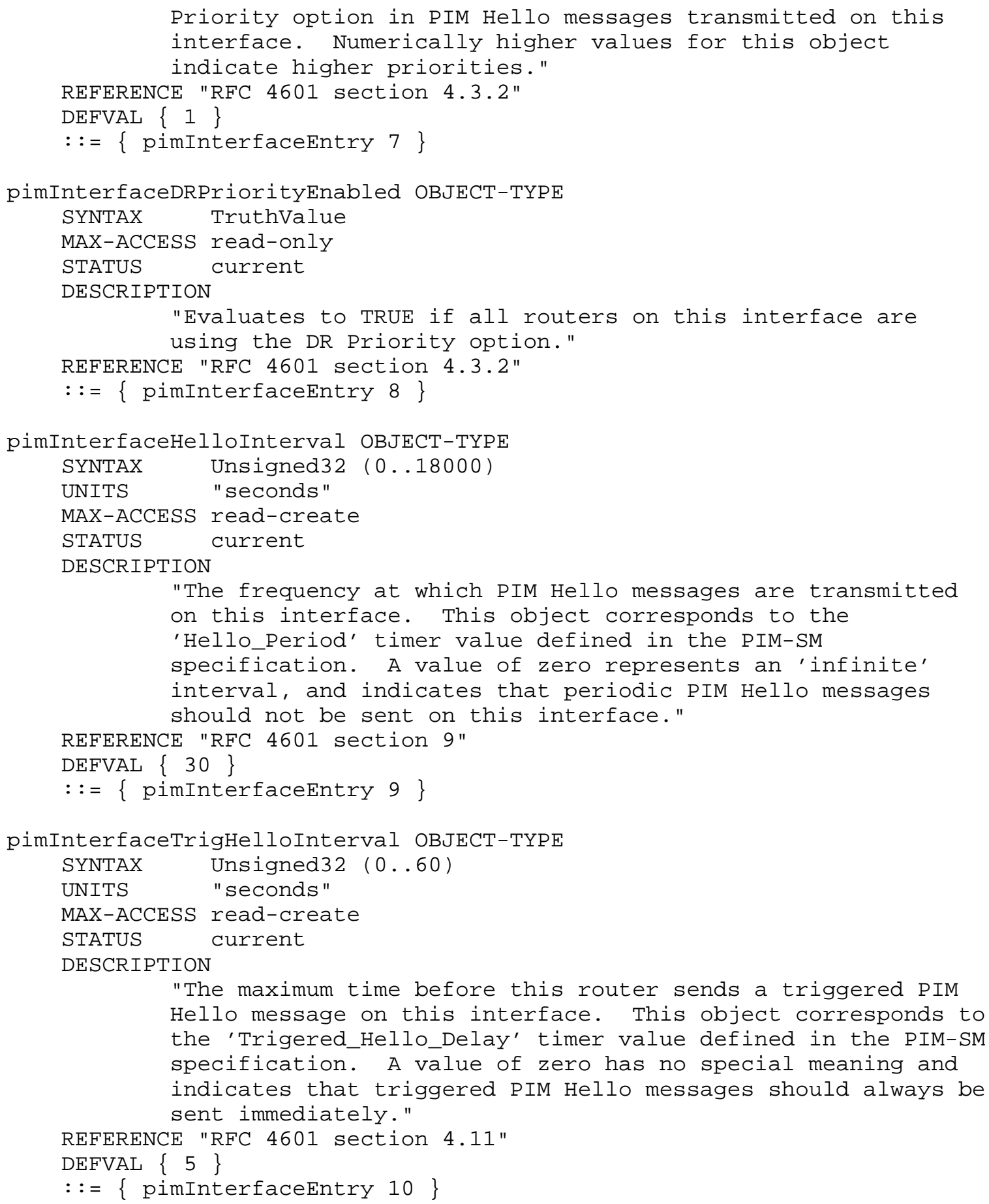




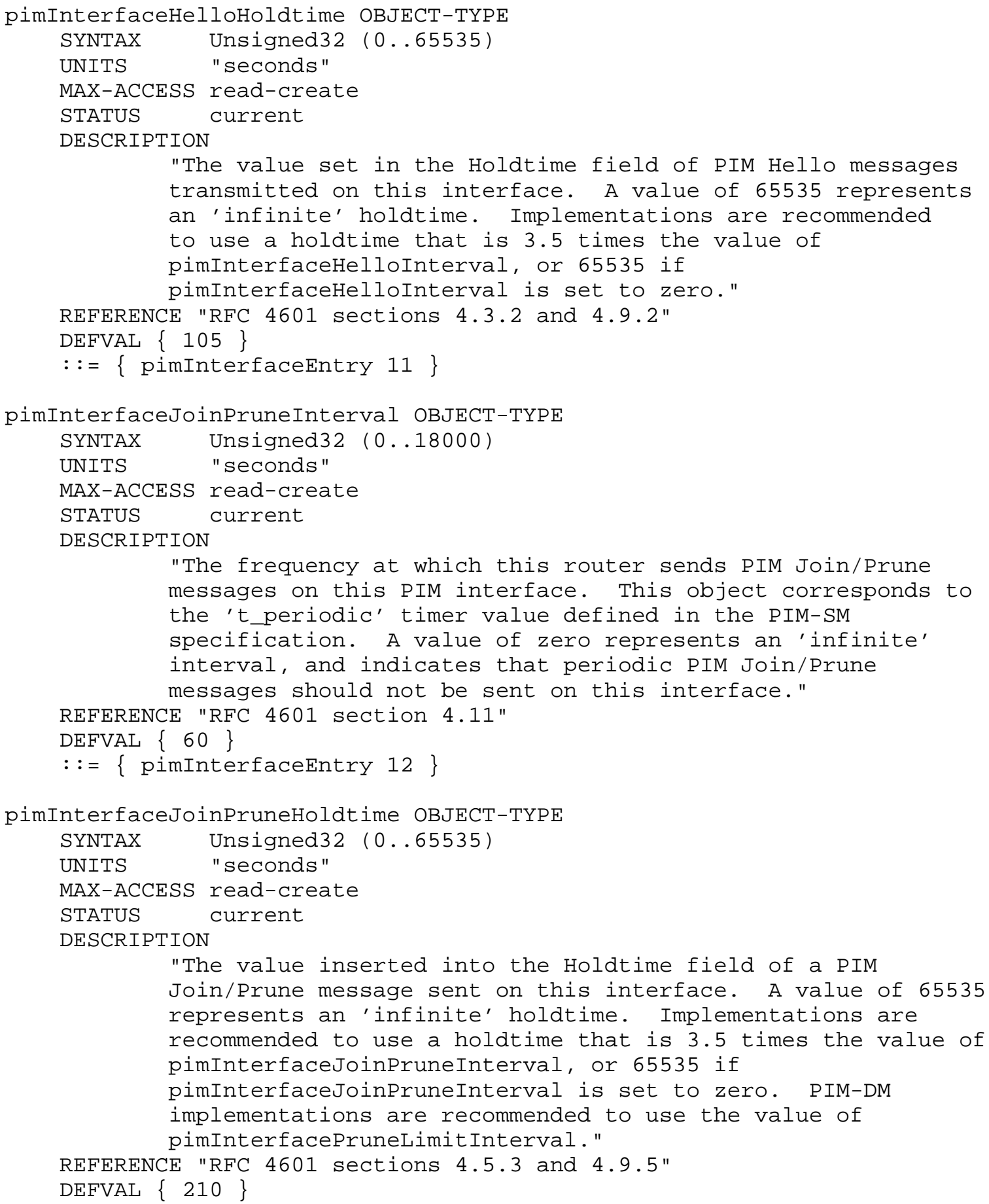




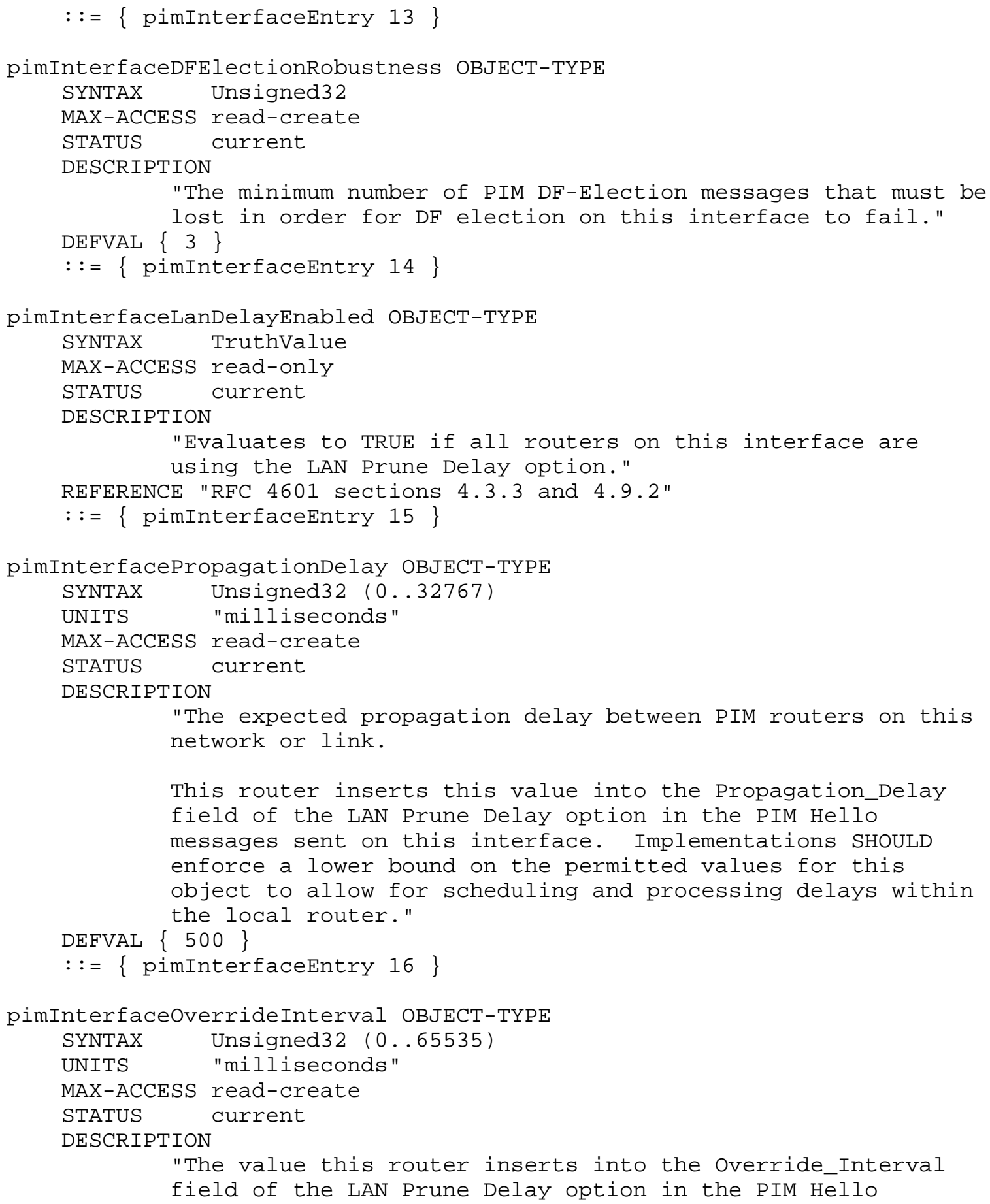




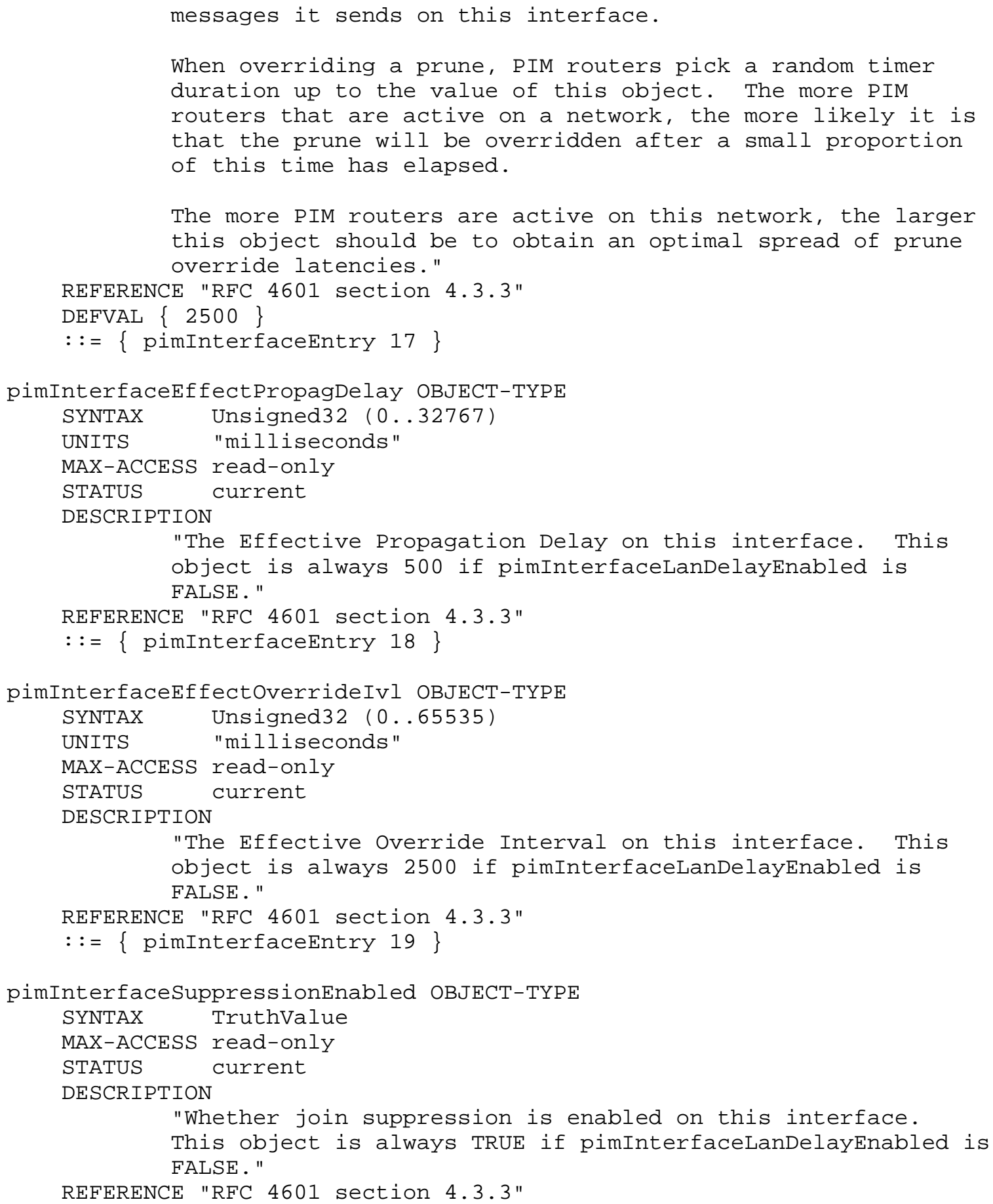




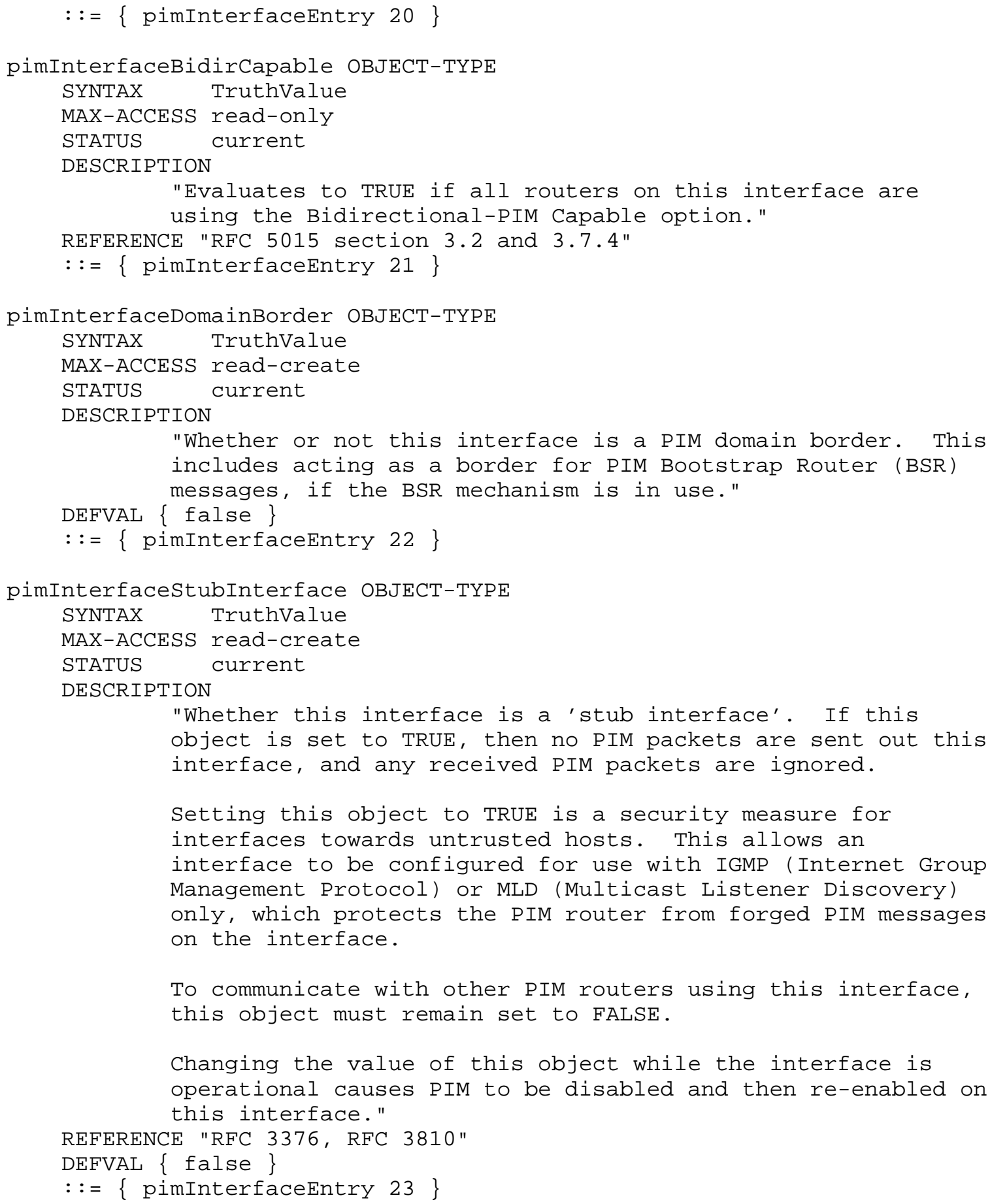




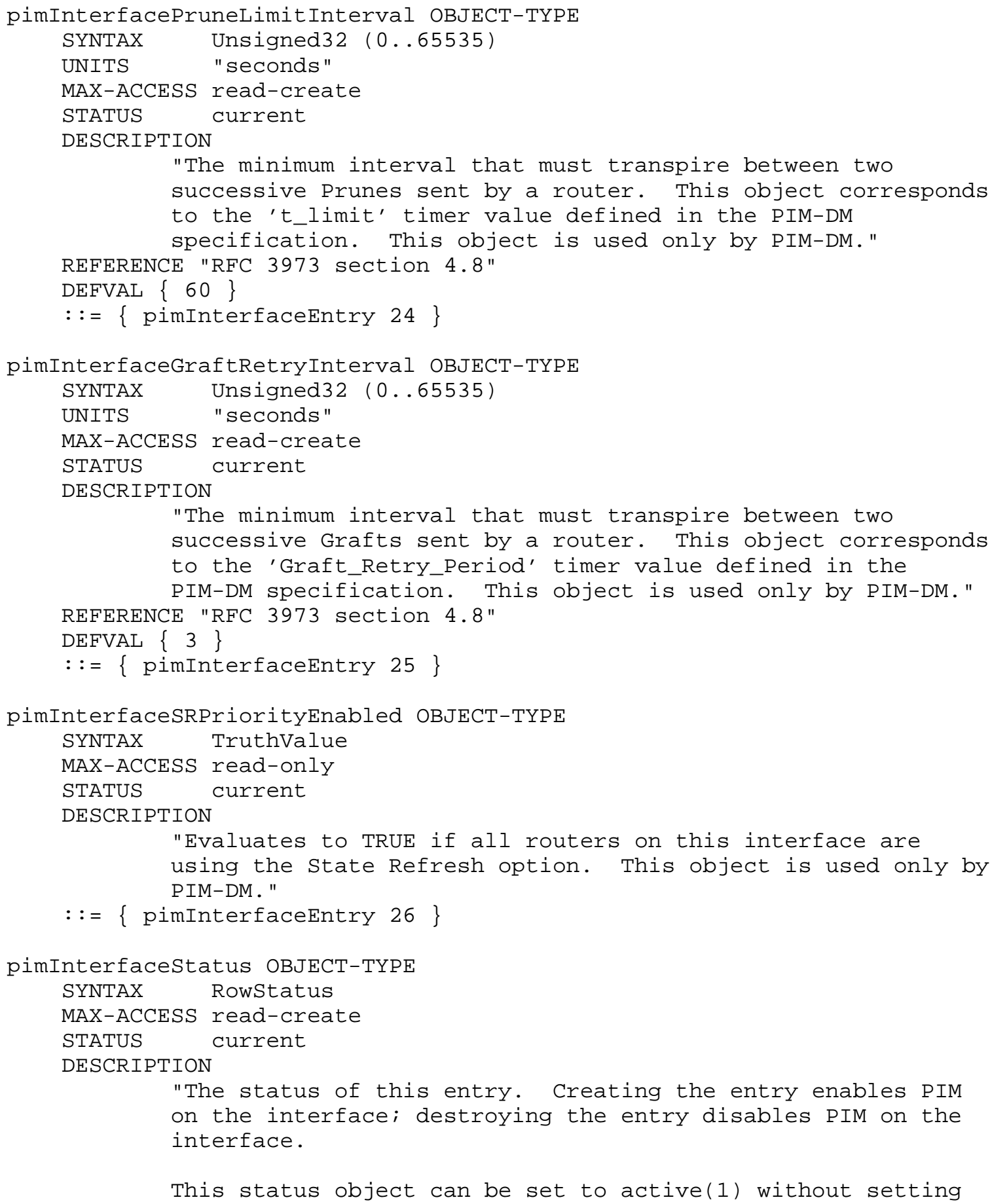




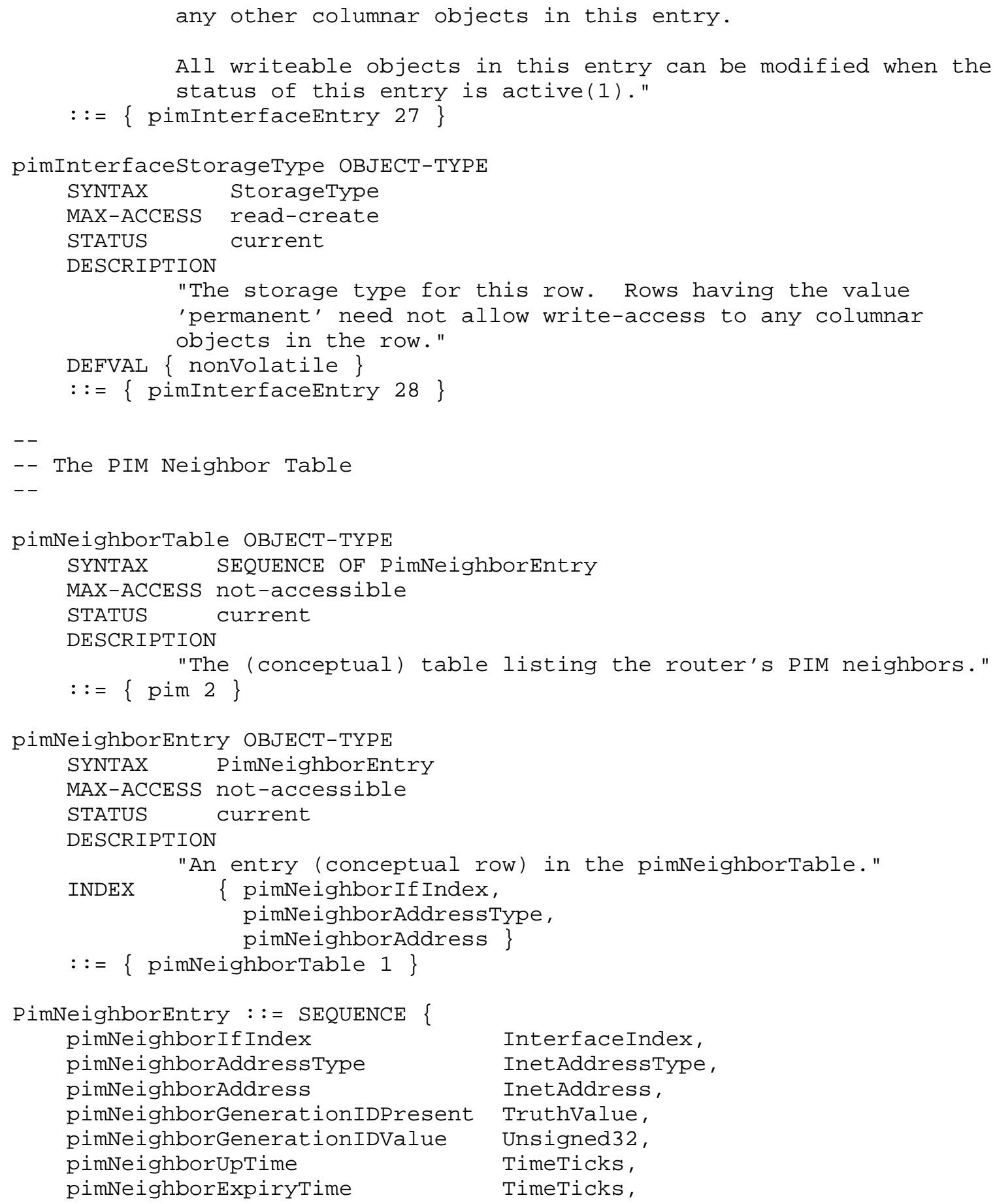

Sivaramu, et al.

Standards Track

[Page 26] 


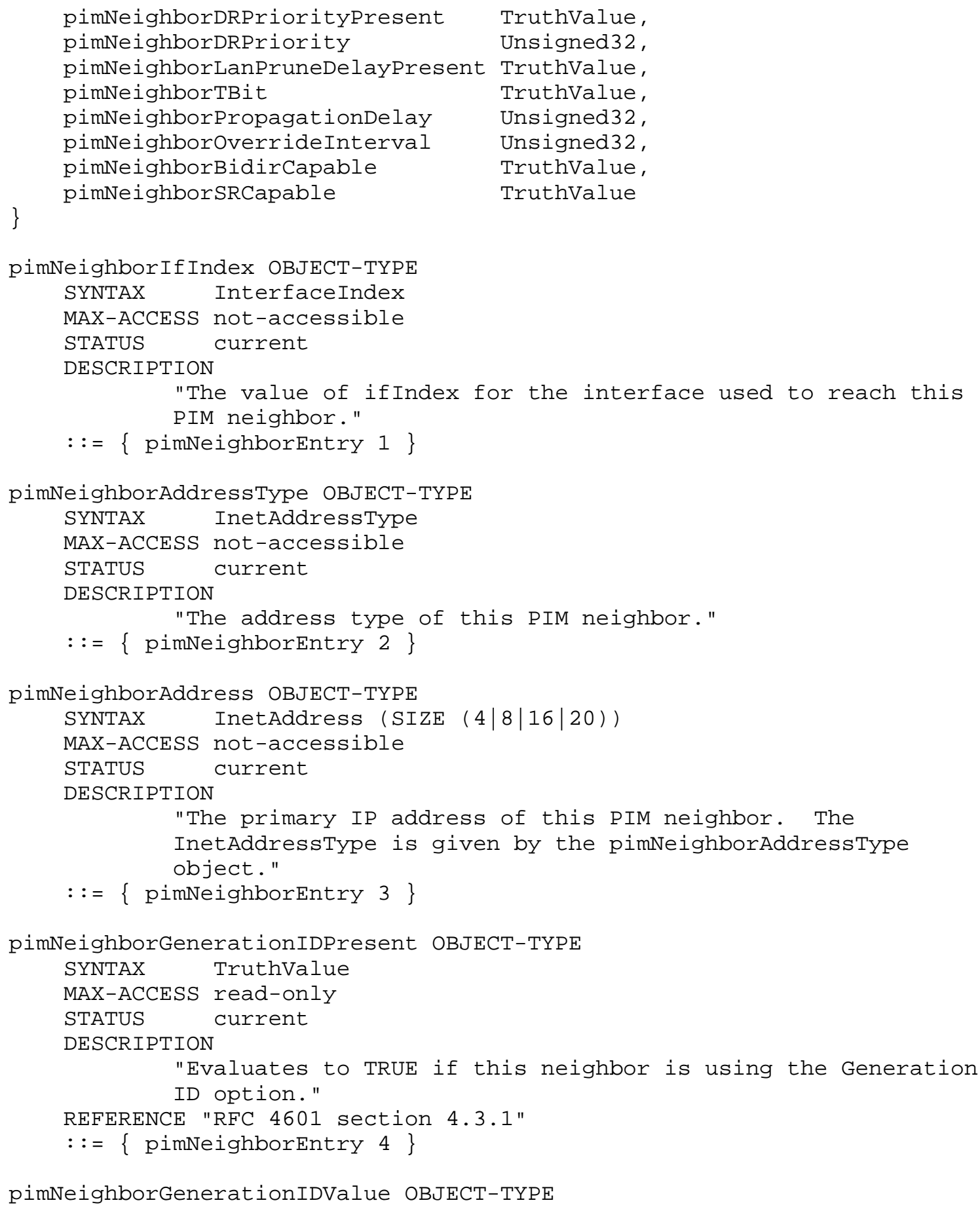




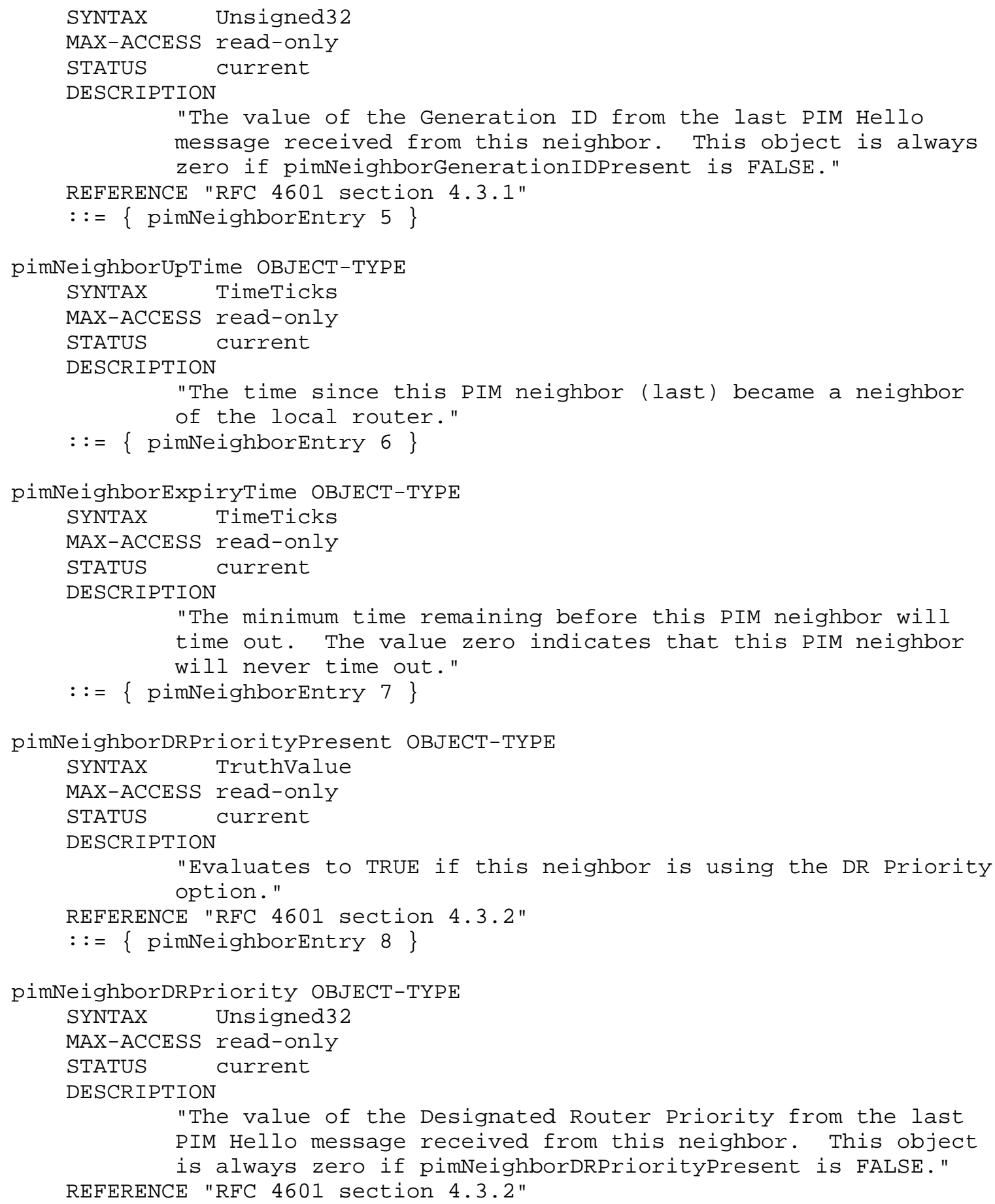


$::=\{$ pimNeighborEntry 9$\}$

pimNeighborLanPruneDelayPresent OBJECT-TYPE

SYNTAX TruthValue

MAX-ACCESS read-only

STATUS current

DESCRIPTION

"Evaluates to TRUE if this neighbor is using the LAN Prune Delay option."

REFERENCE "RFC 4601 section 4.3.3"

$::=\{$ pimNeighborEntry 10$\}$

pimNeighborTBit OBJECT-TYPE

SYNTAX TruthValue

MAX-ACCESS read-only

STATUS current

DESCRIPTION

"Whether the $\mathrm{T}$ bit was set in the LAN Prune Delay option

received from this neighbor. The $\mathrm{T}$ bit specifies the ability of the neighbor to disable join suppression. This object is always TRUE if pimNeighborLanPruneDelayPresent is FALSE."

REFERENCE "RFC 4601 section 4.3.3"

$::=\{$ pimNeighborEntry 11$\}$

pimNeighborPropagationDelay OBJECT-TYPE

SYNTAX Unsigned32 (0..32767)

MAX-ACCESS read-only

STATUS current

DESCRIPTION

"The value of the Propagation_Delay field of the LAN Prune Delay option received from this neighbor. This object is always zero if pimNeighborLanPruneDelayPresent is FALSE." REFERENCE "RFC 4601 section 4.3.3"

$::=\{$ pimNeighborEntry 12$\}$

pimNeighborOverrideInterval OBJECT-TYPE

SYNTAX Unsigned32 (0..65535)

MAX-ACCESS read-only

STATUS current

DESCRIPTION

"The value of the Override_Interval field of the LAN Prune Delay option received from this neighbor. This object is always zero if pimNeighborLanPruneDelayPresent is FALSE." REFERENCE "RFC 4601 section 4.3.3" $::=\{$ pimNeighborEntry 13$\}$

pimNeighborBidirCapable OBJECT-TYPE

Sivaramu, et al.

Standards Track

[Page 29] 


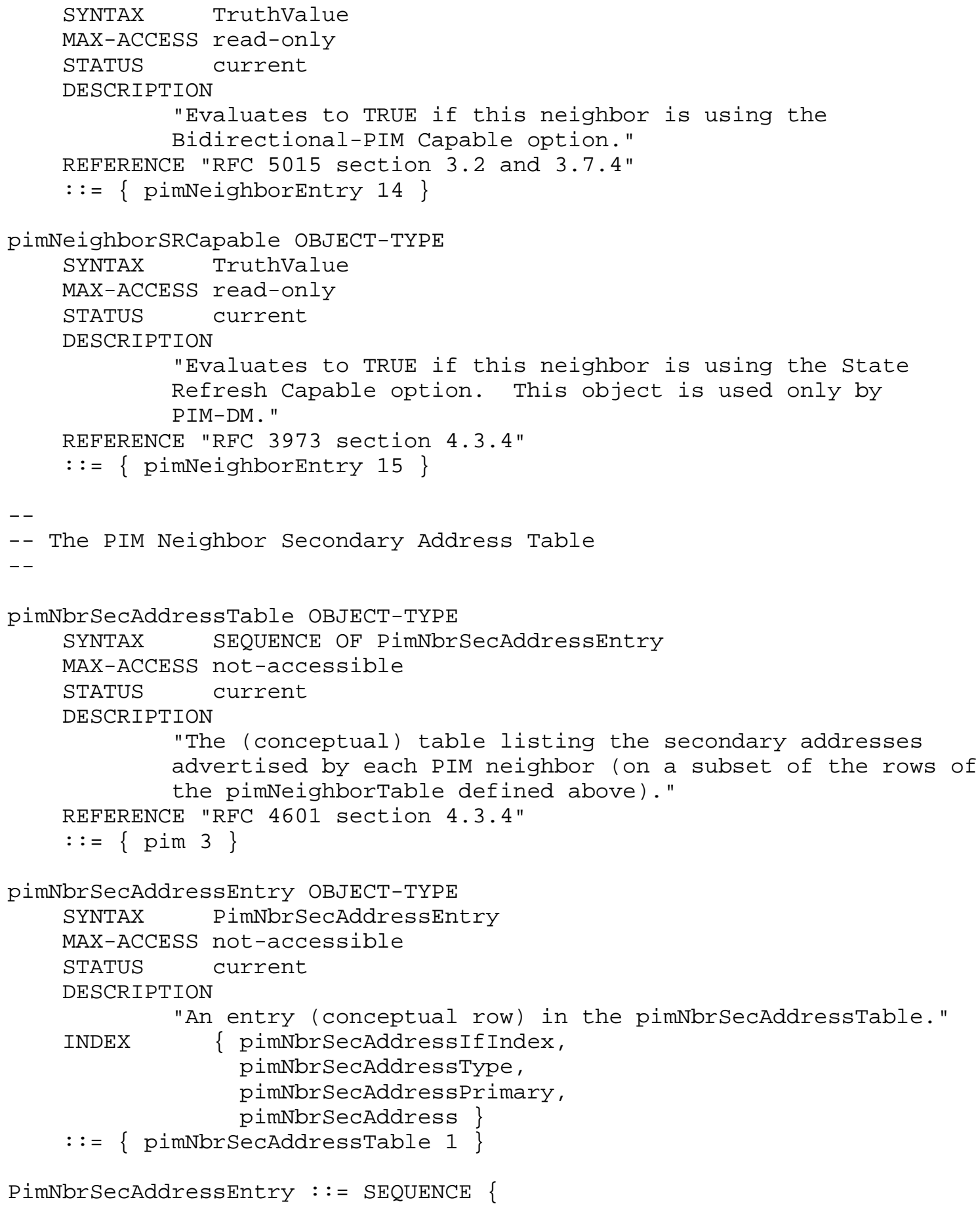




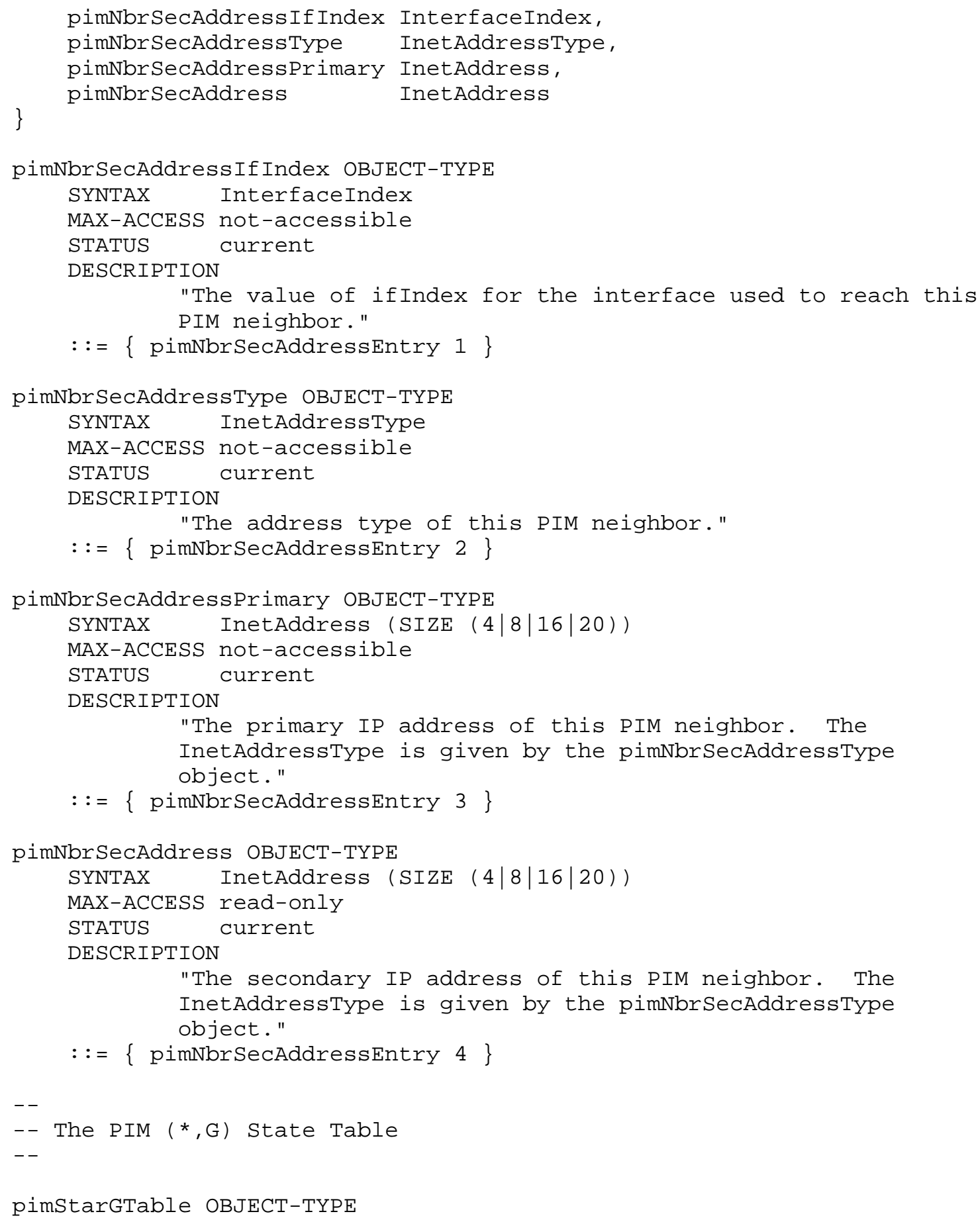




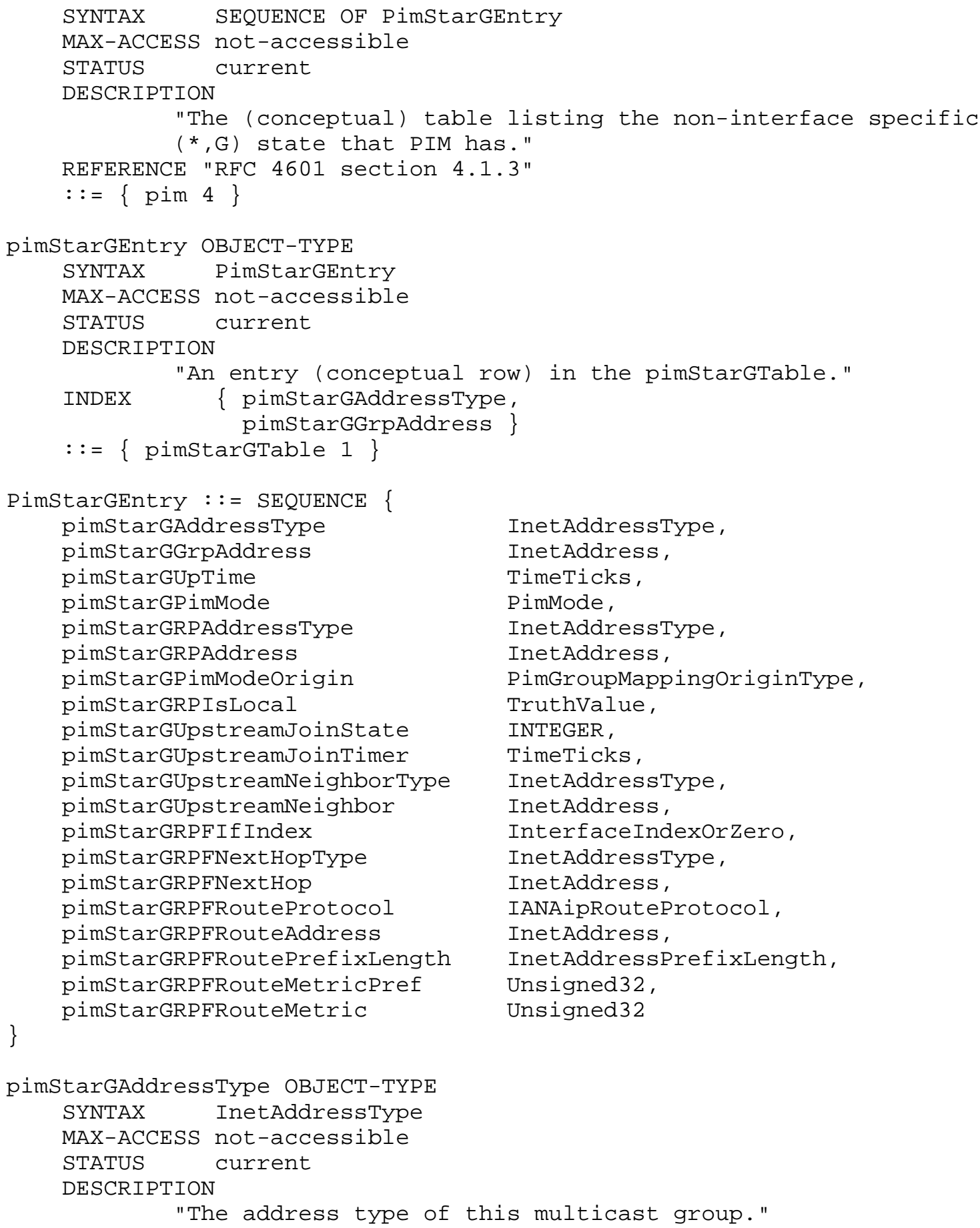




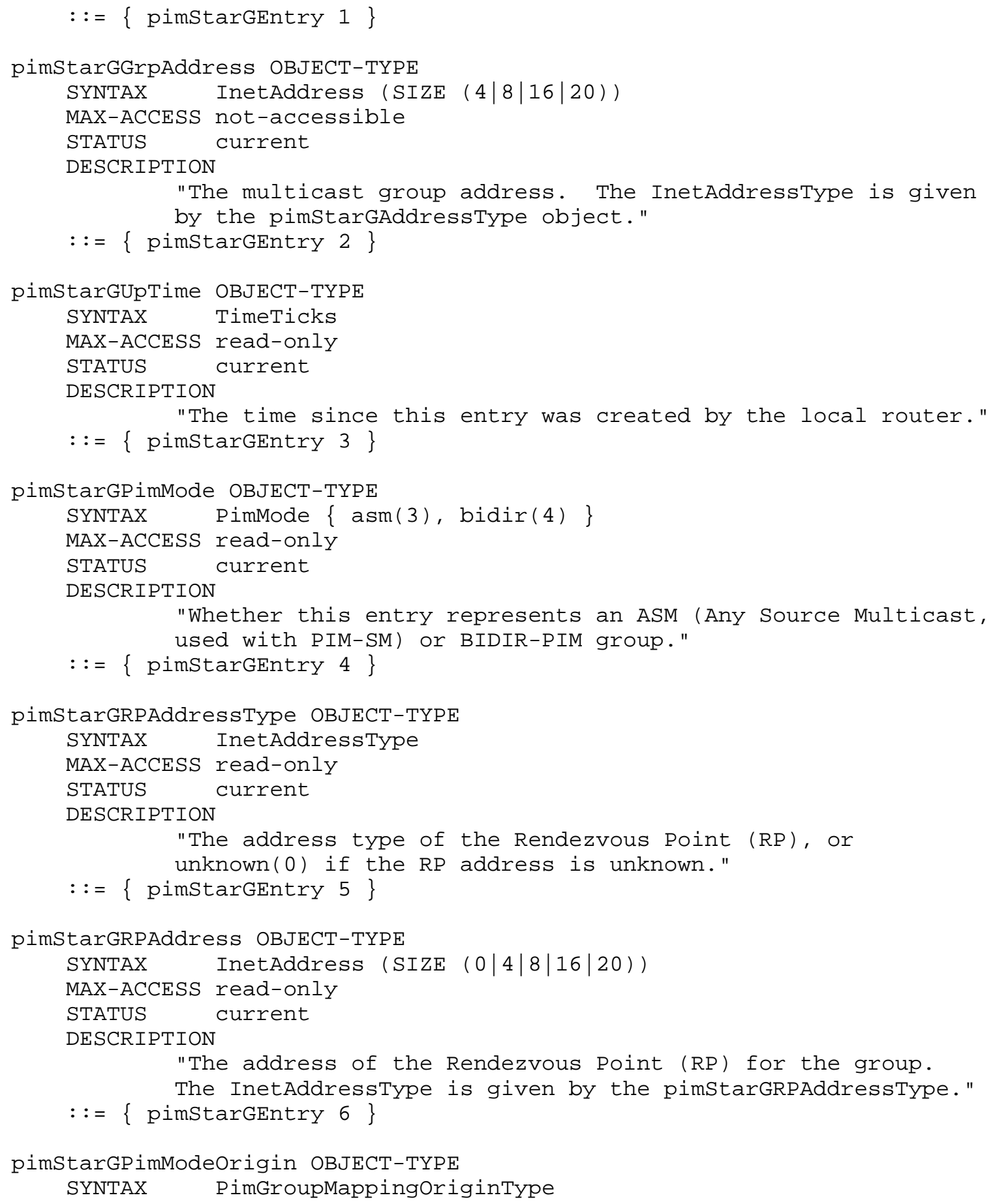




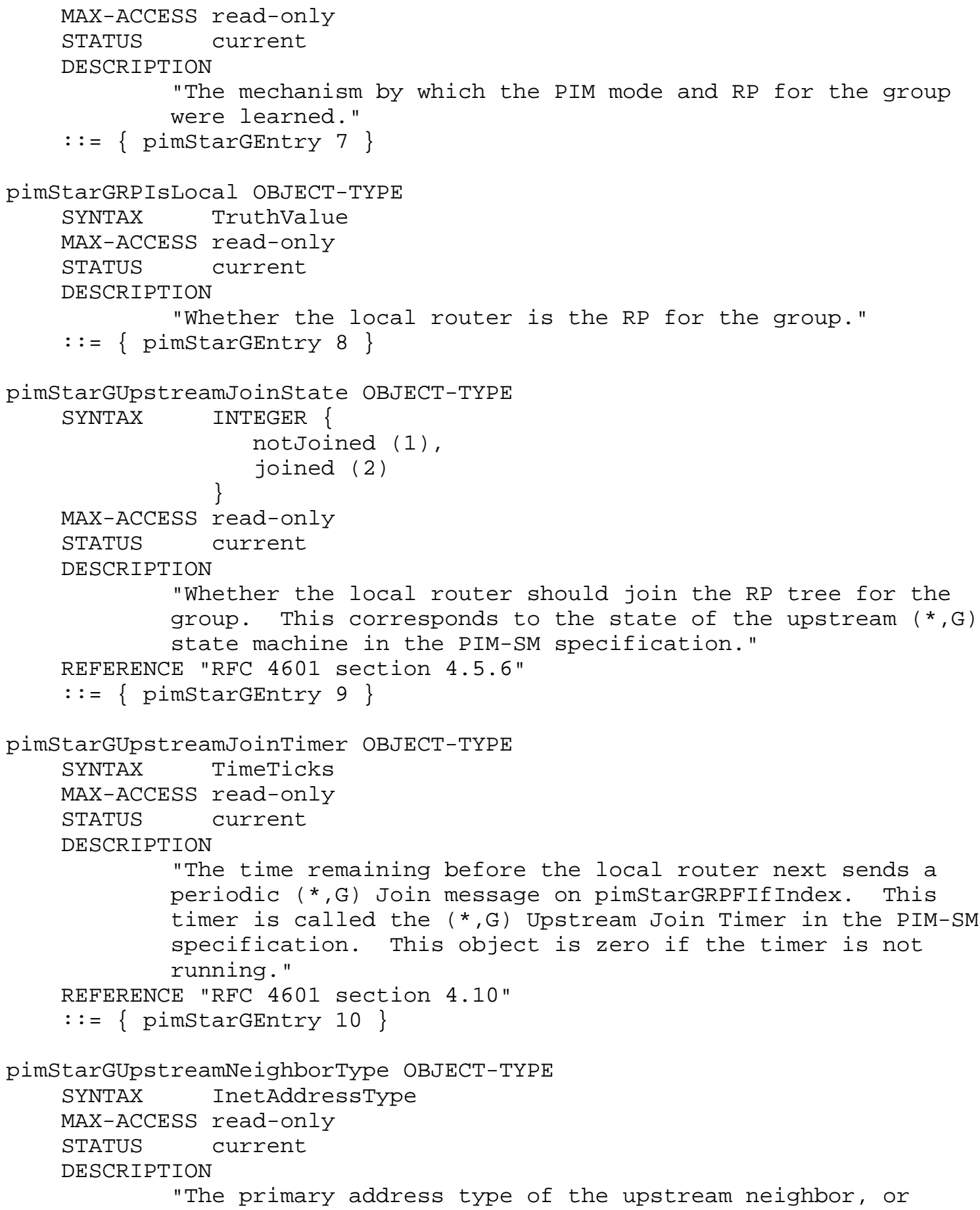




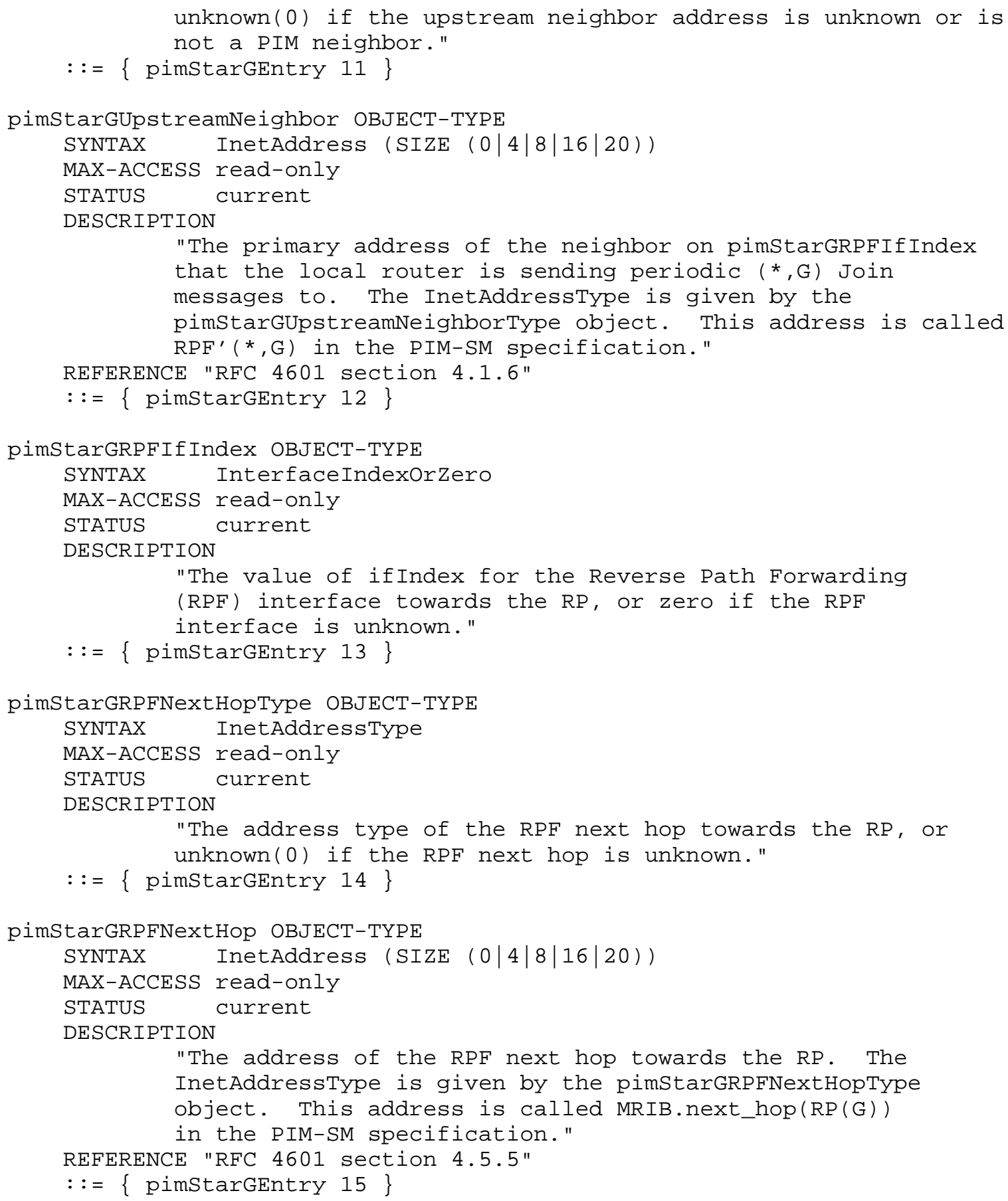




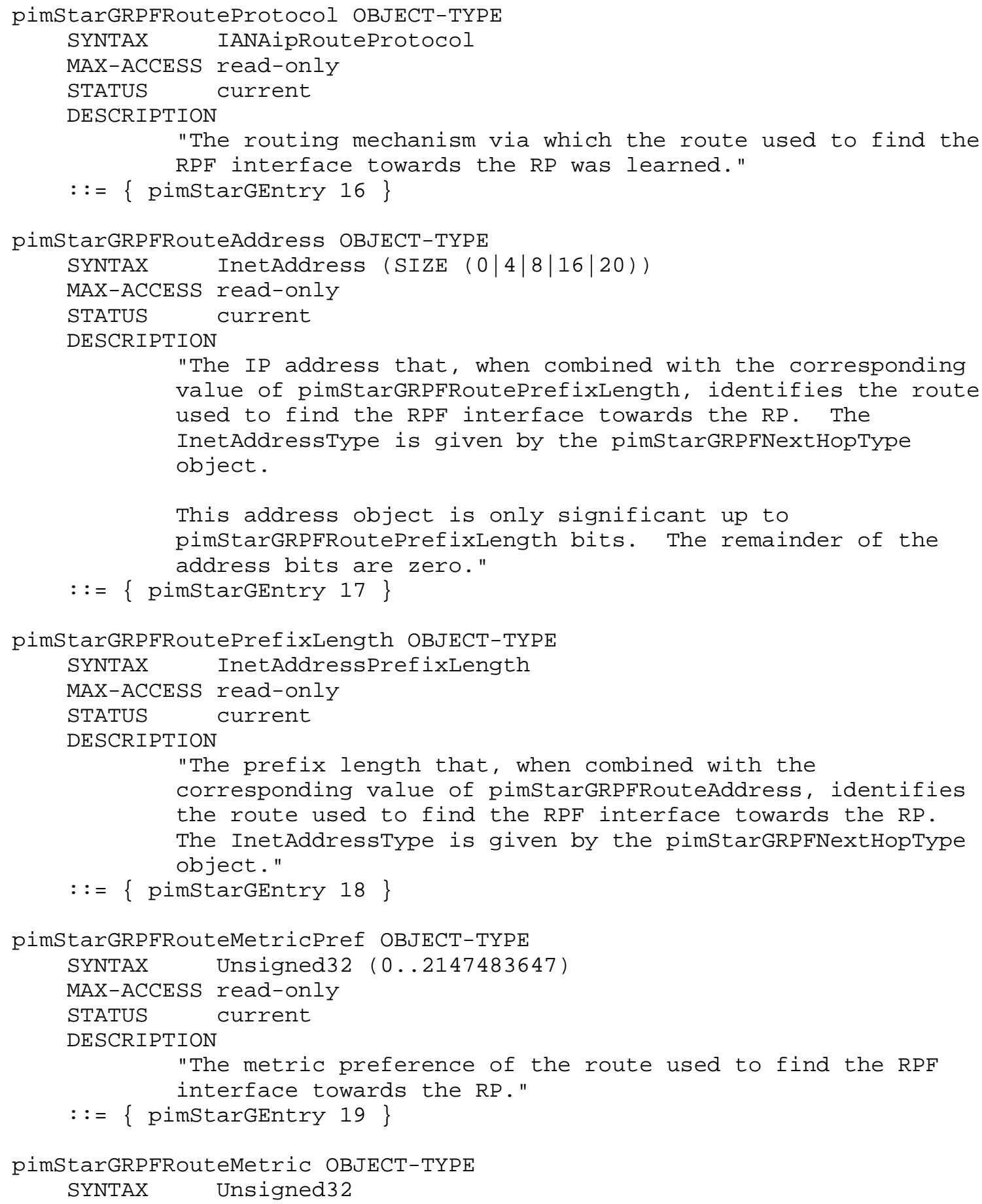




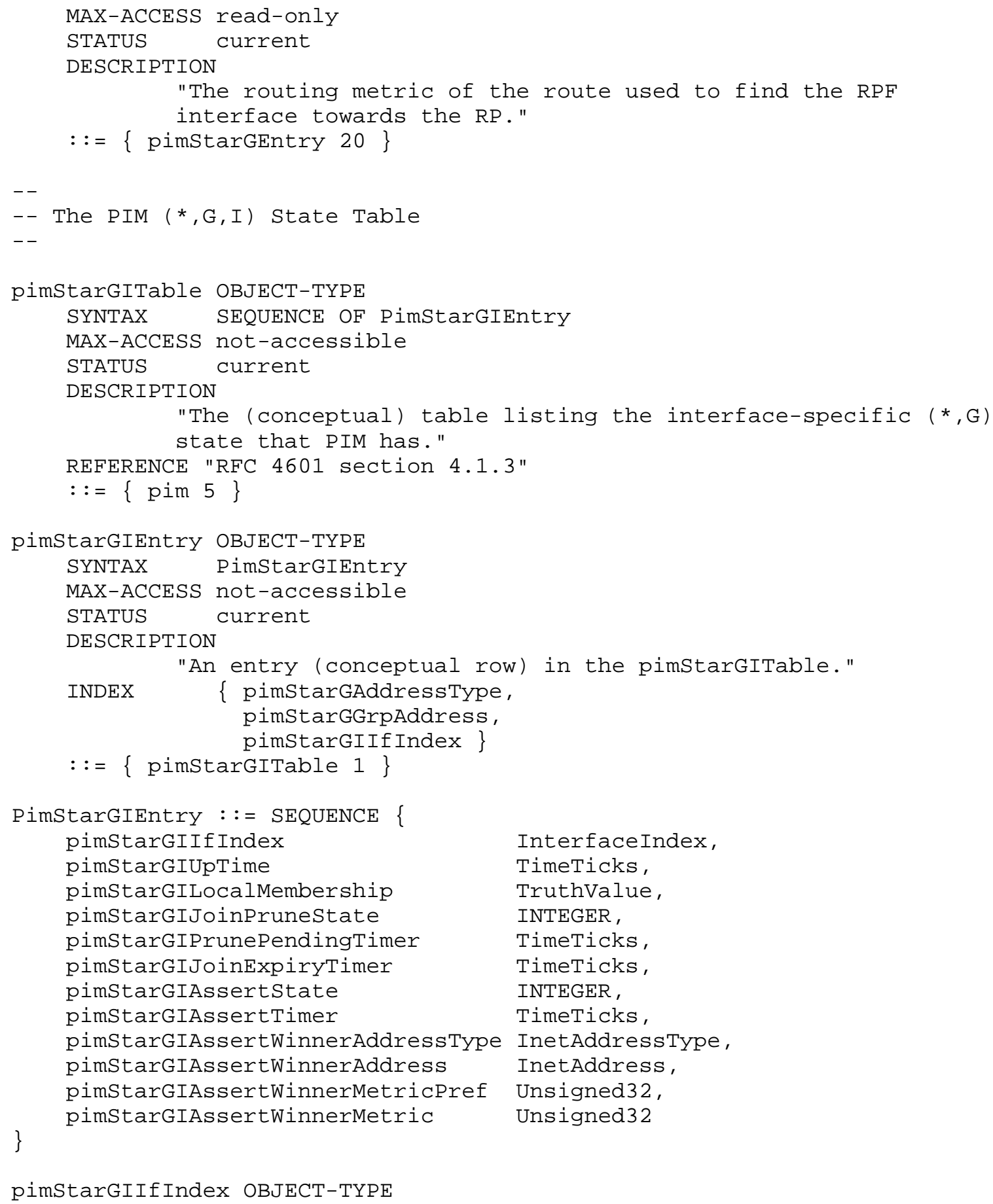




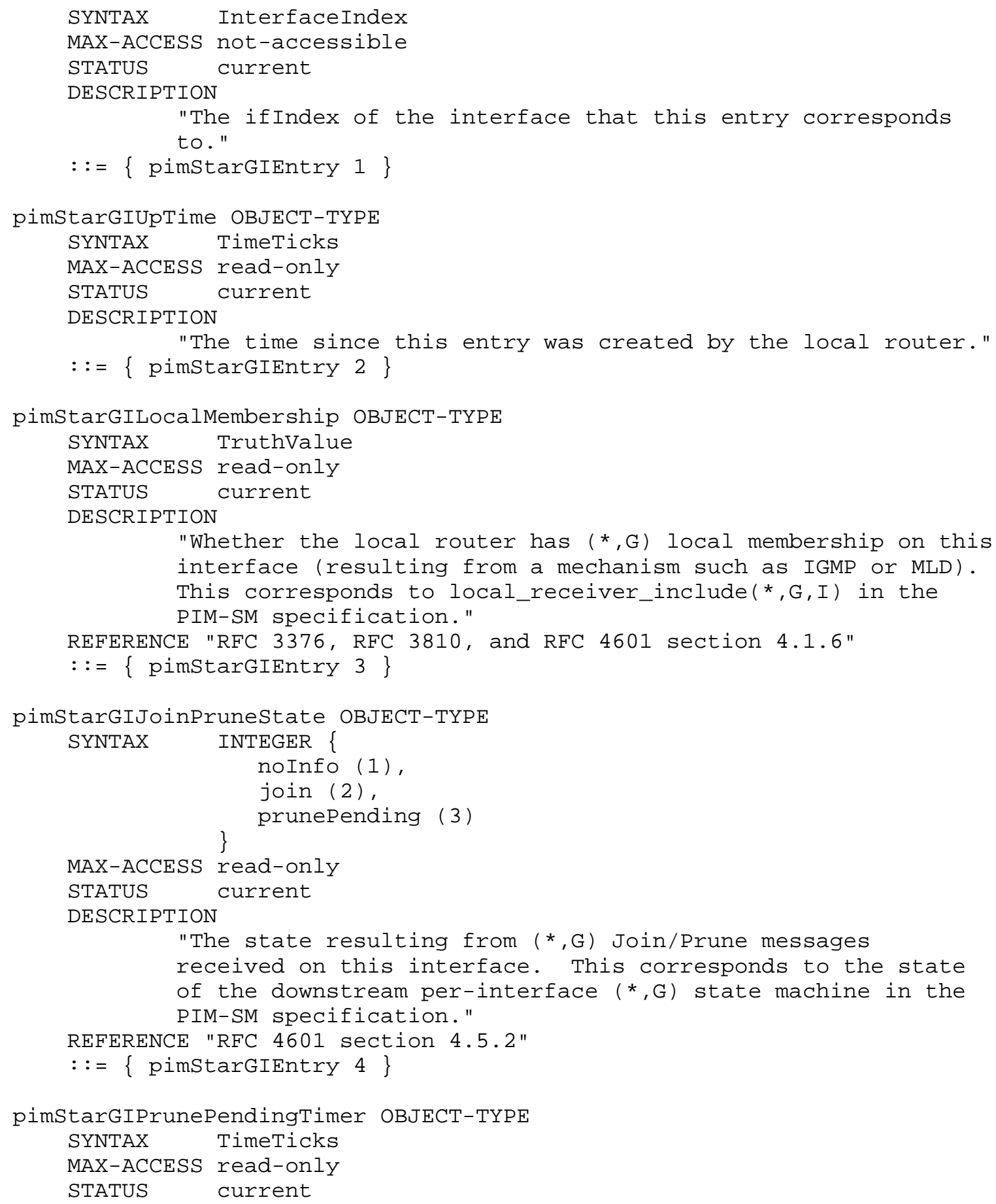




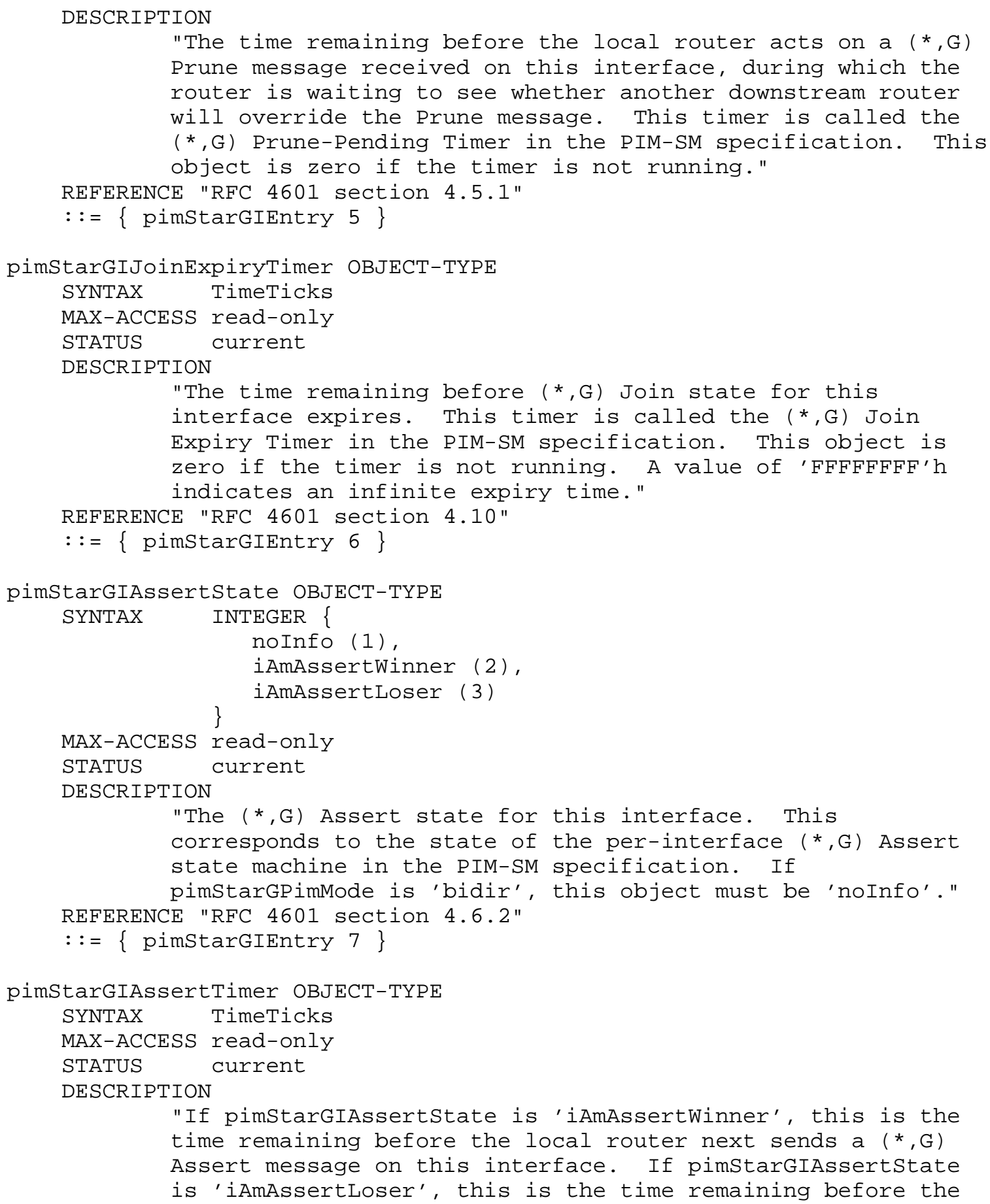




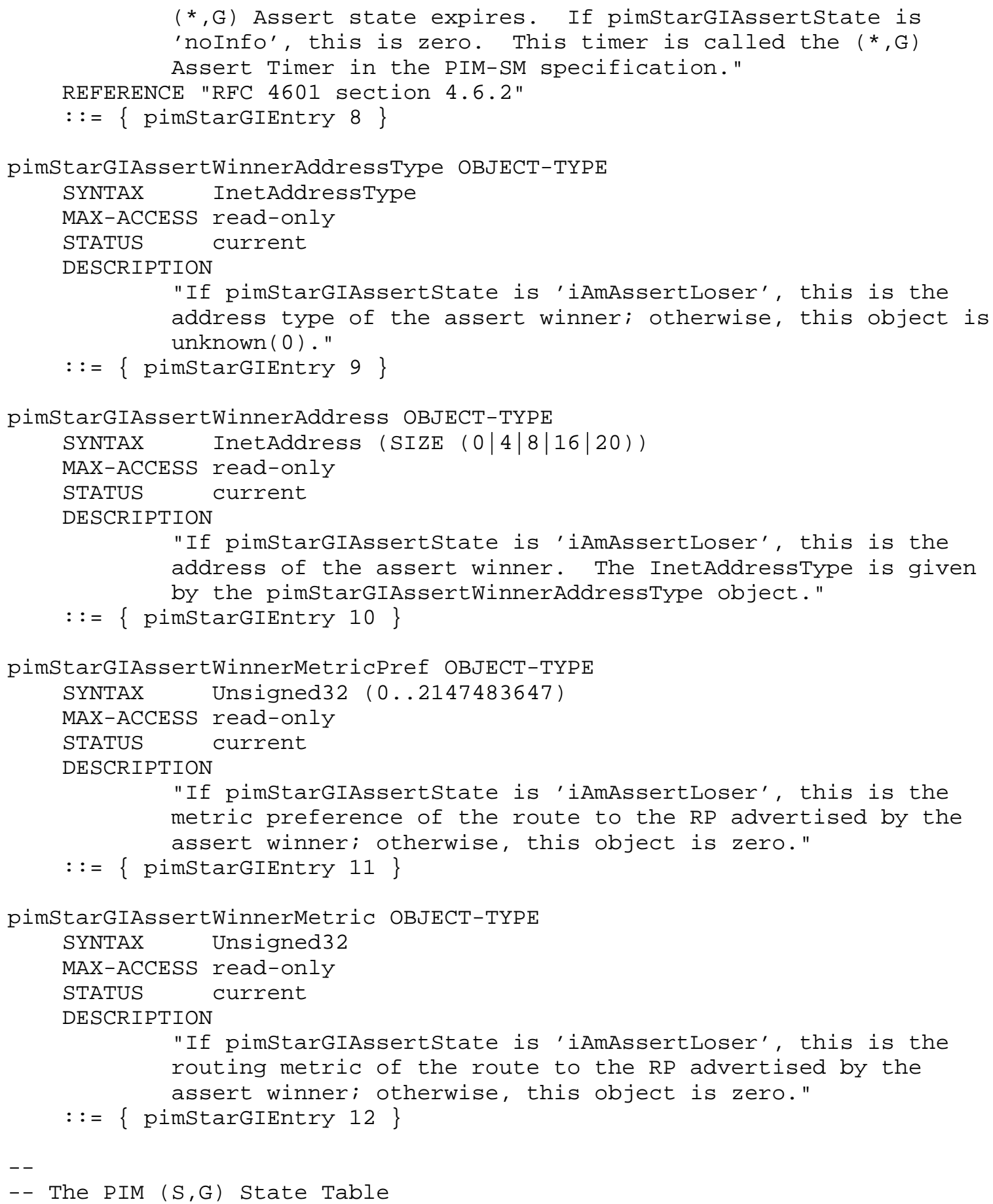




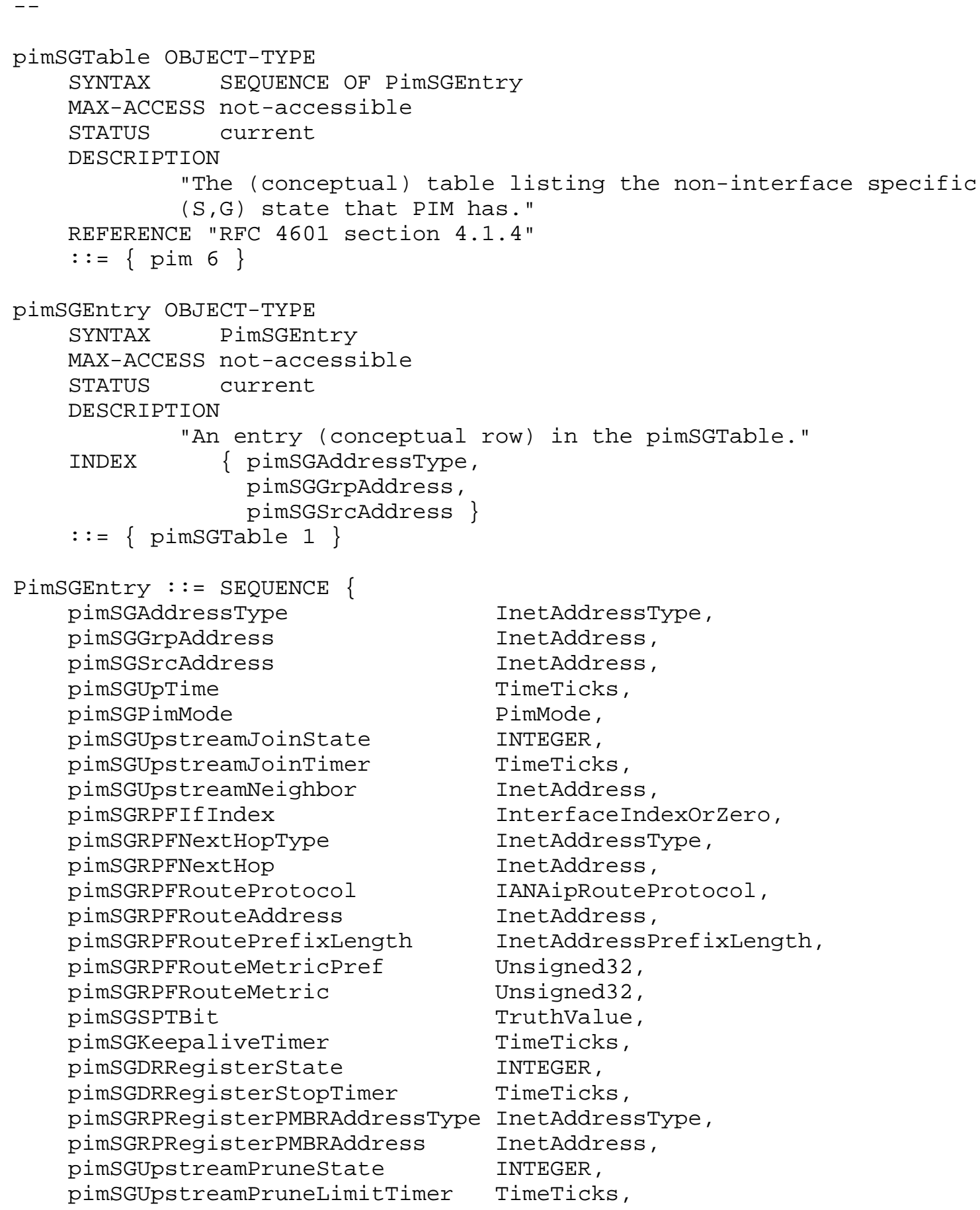




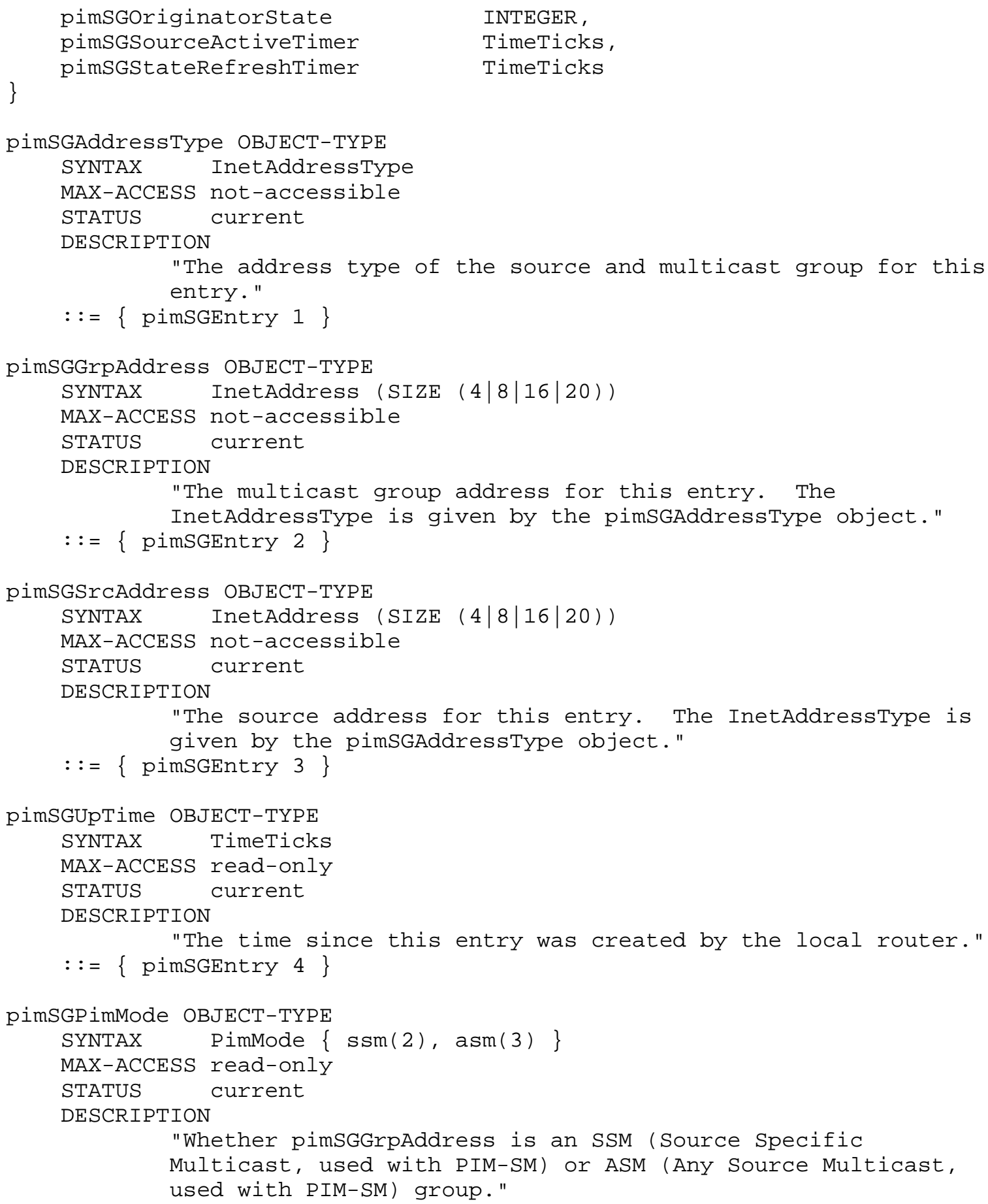




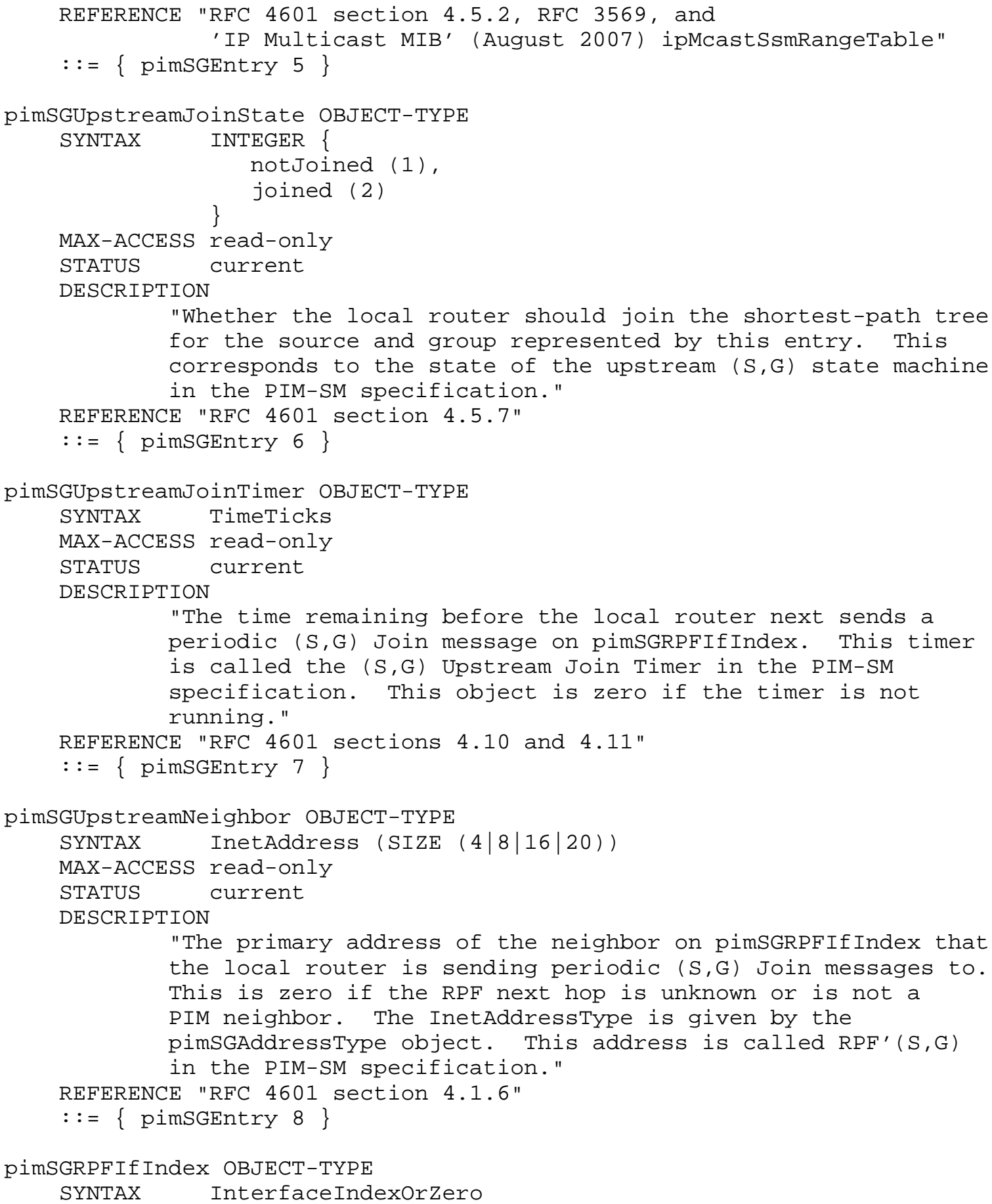




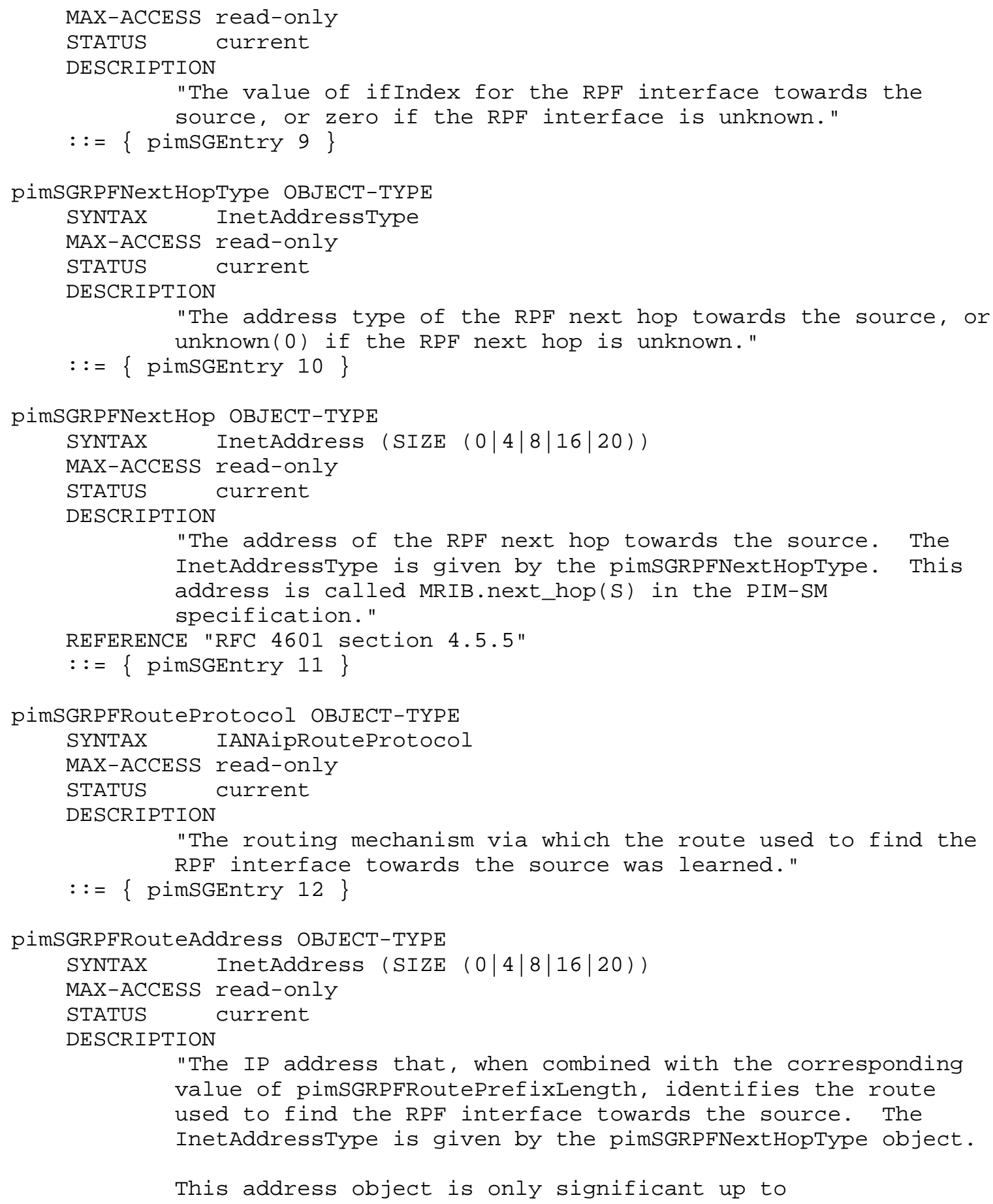




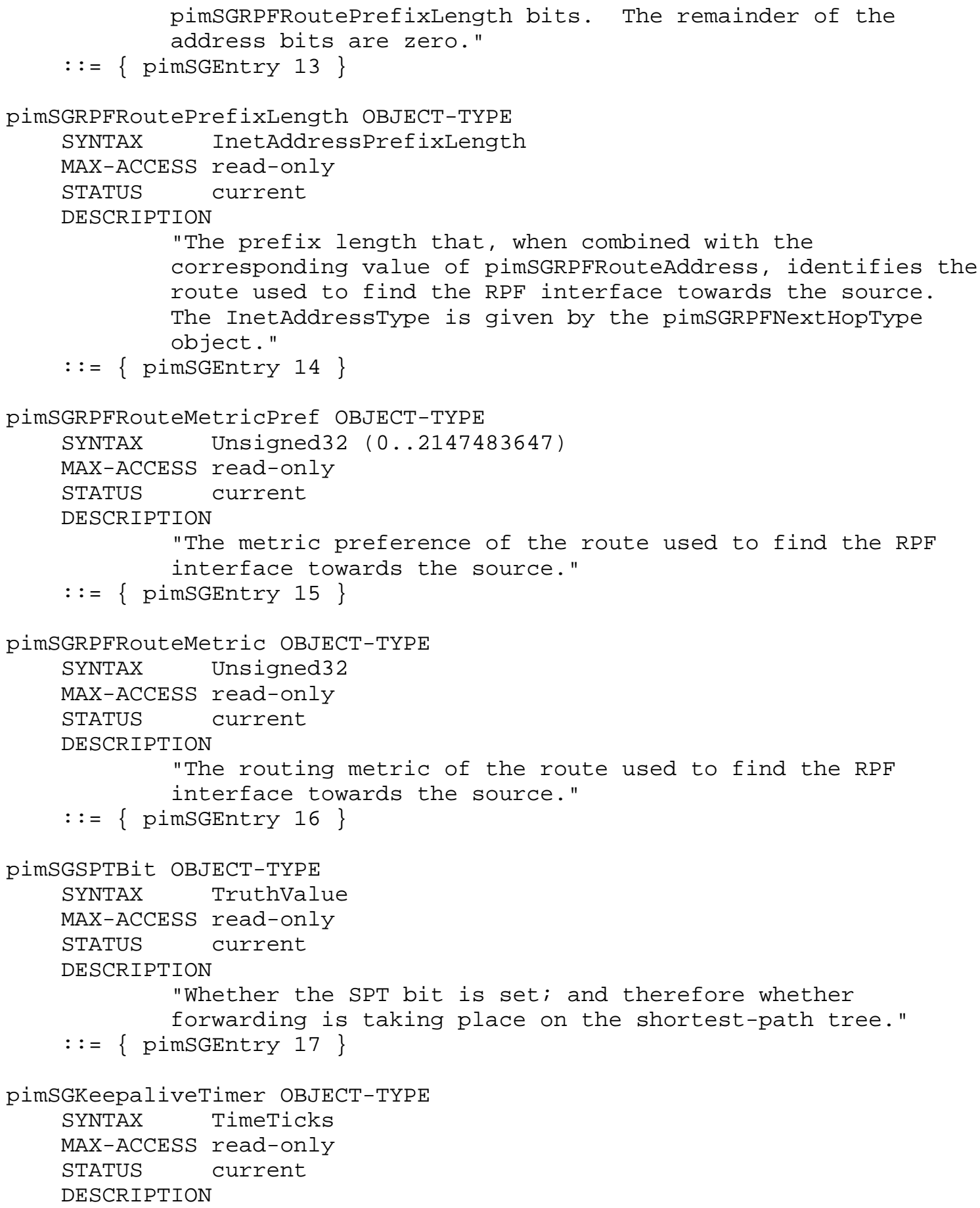




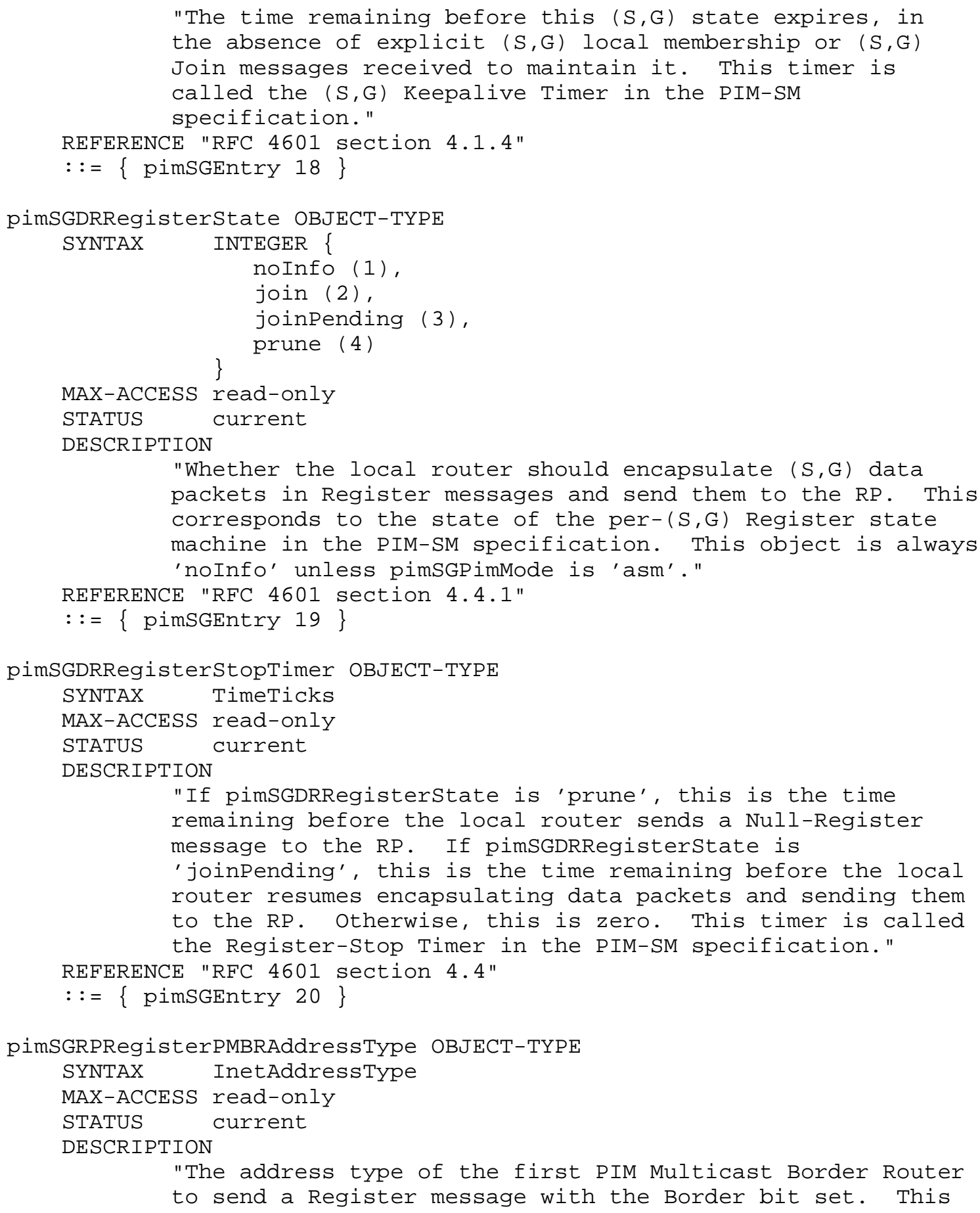




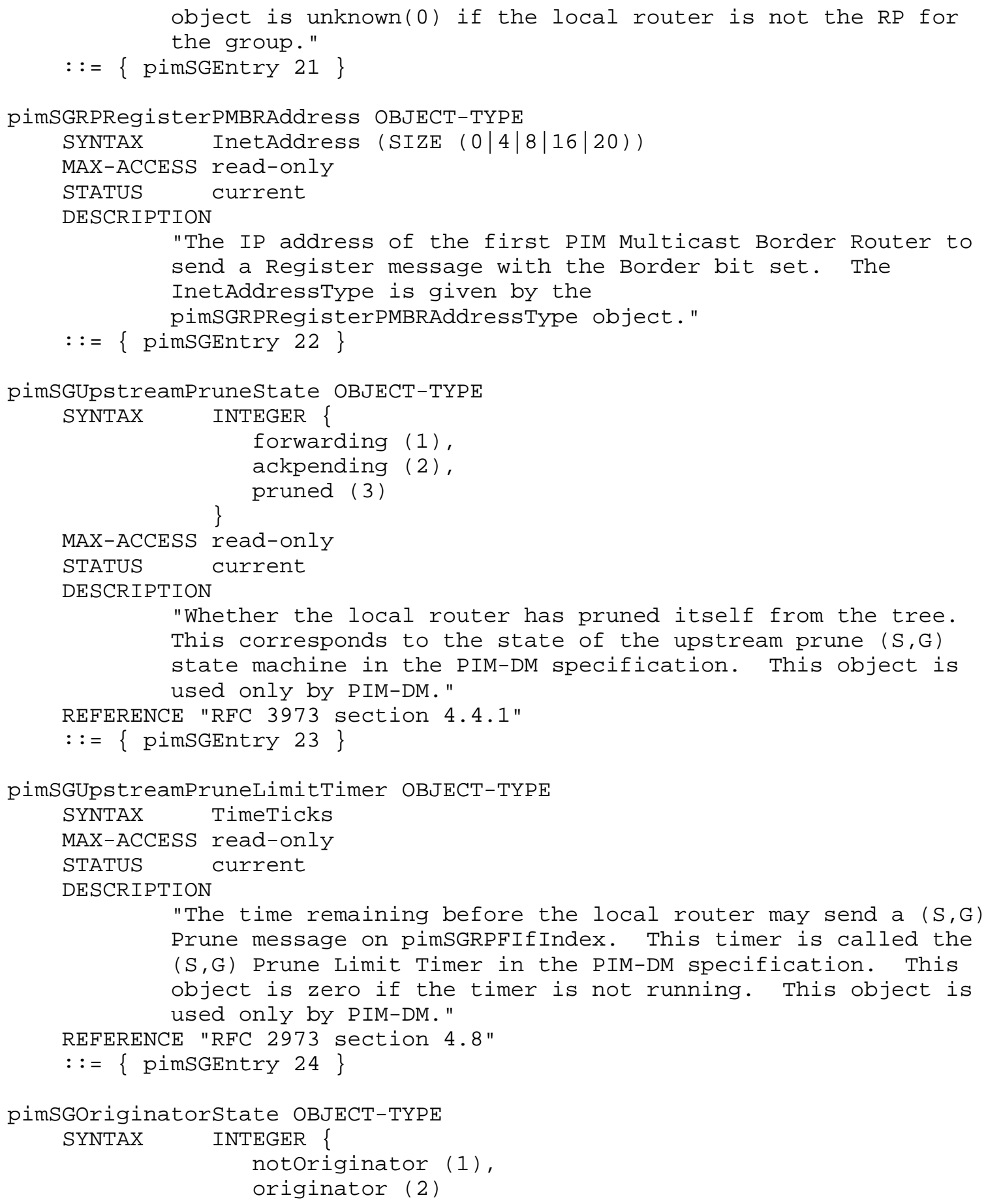




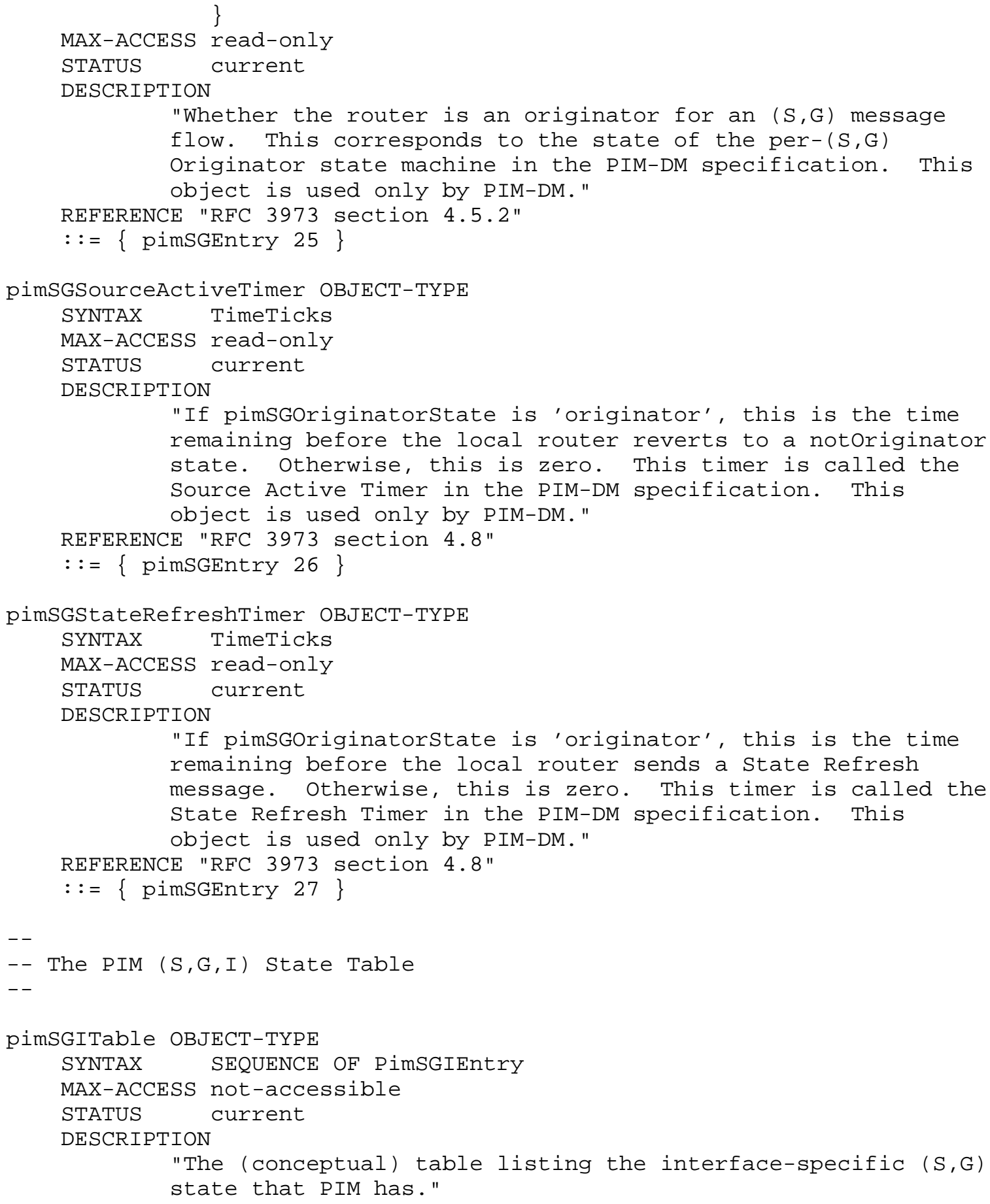




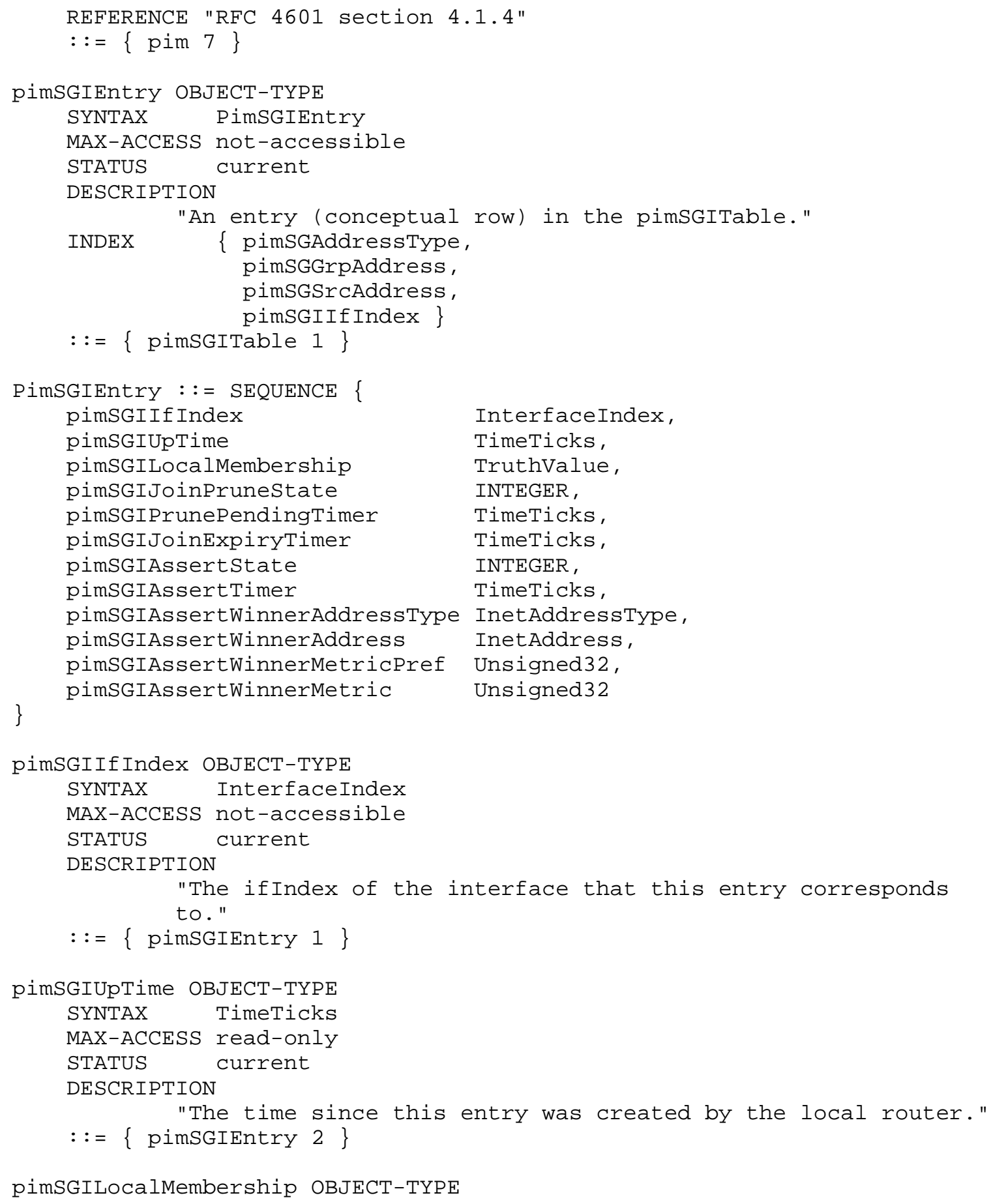




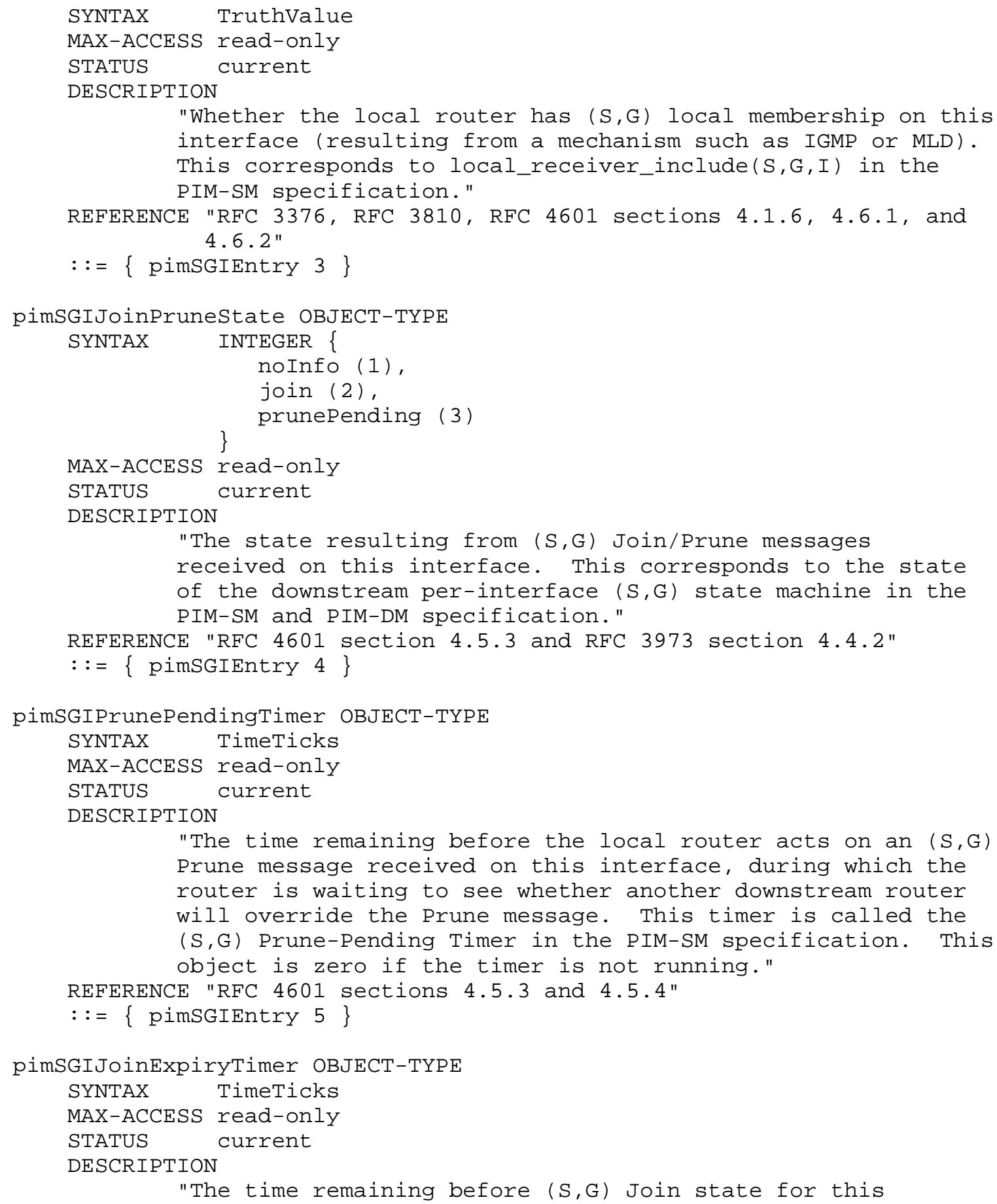




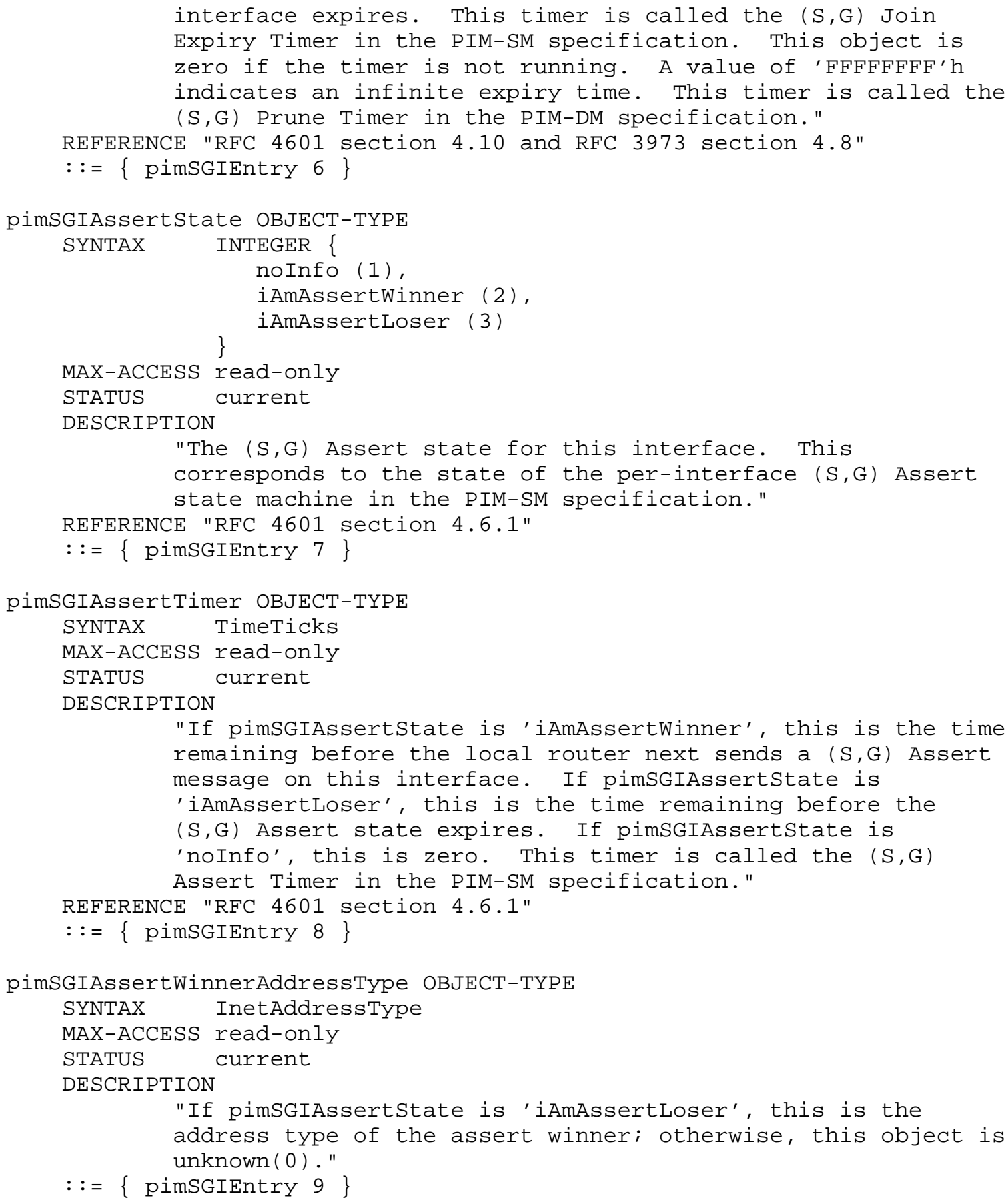




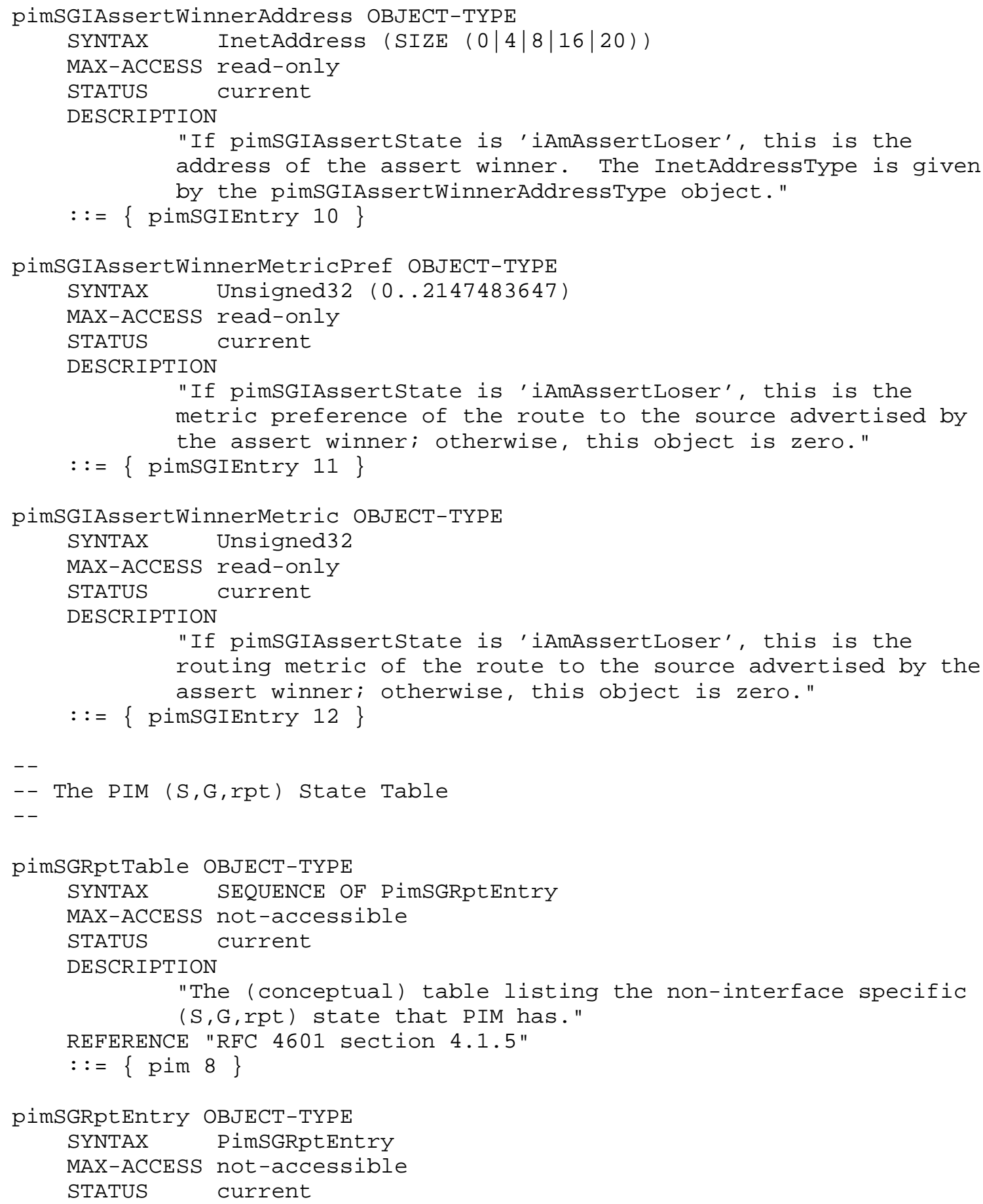




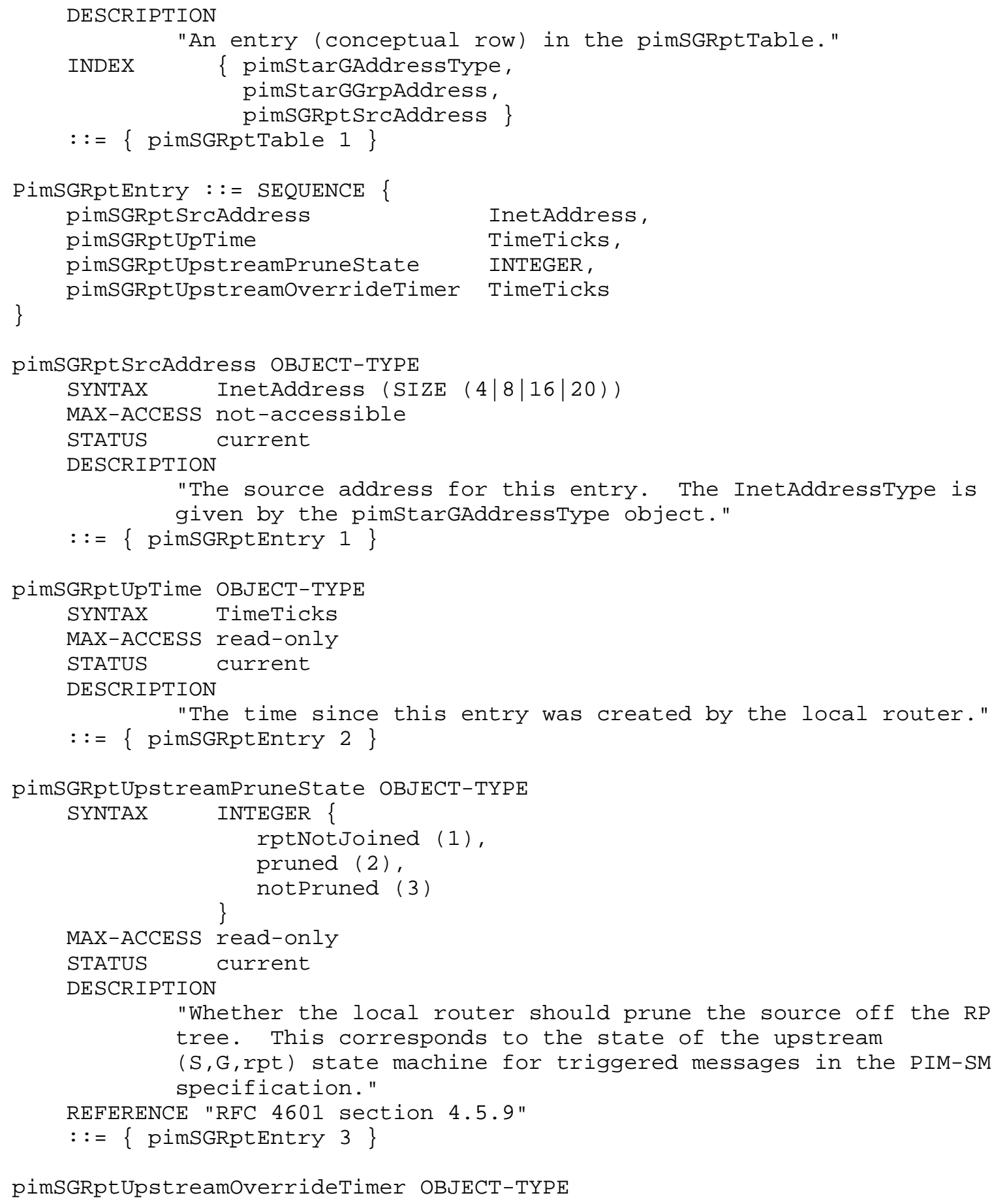




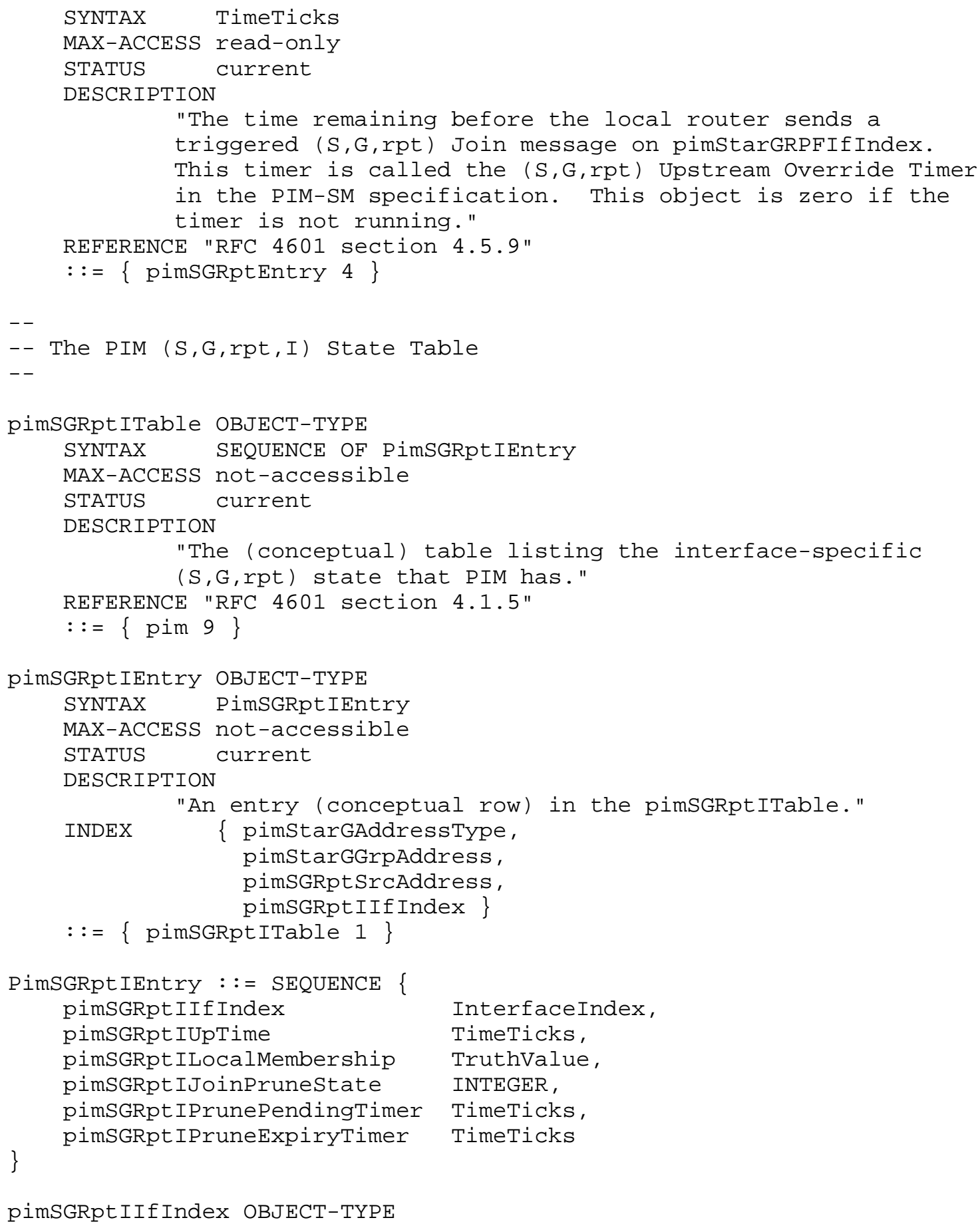




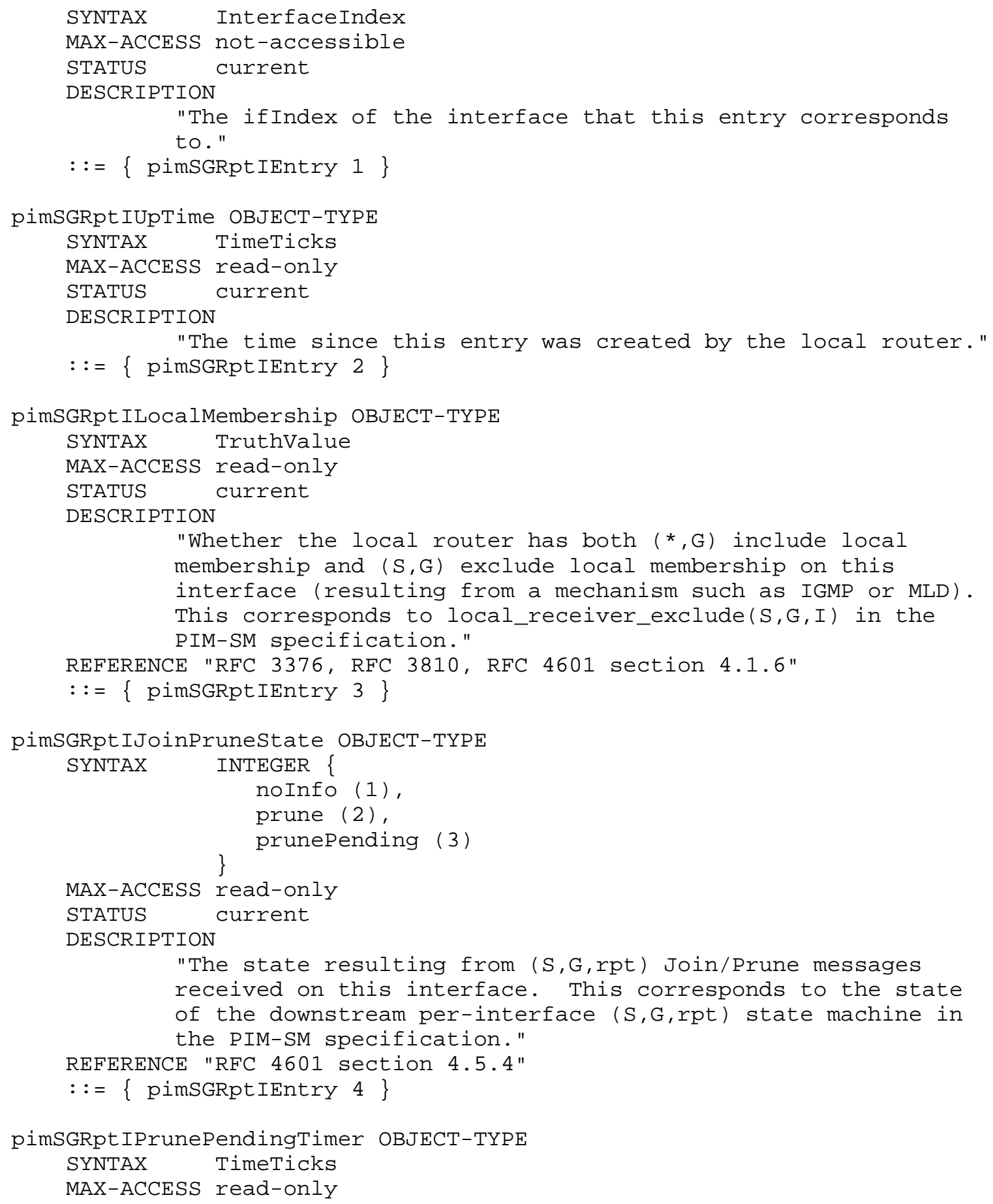




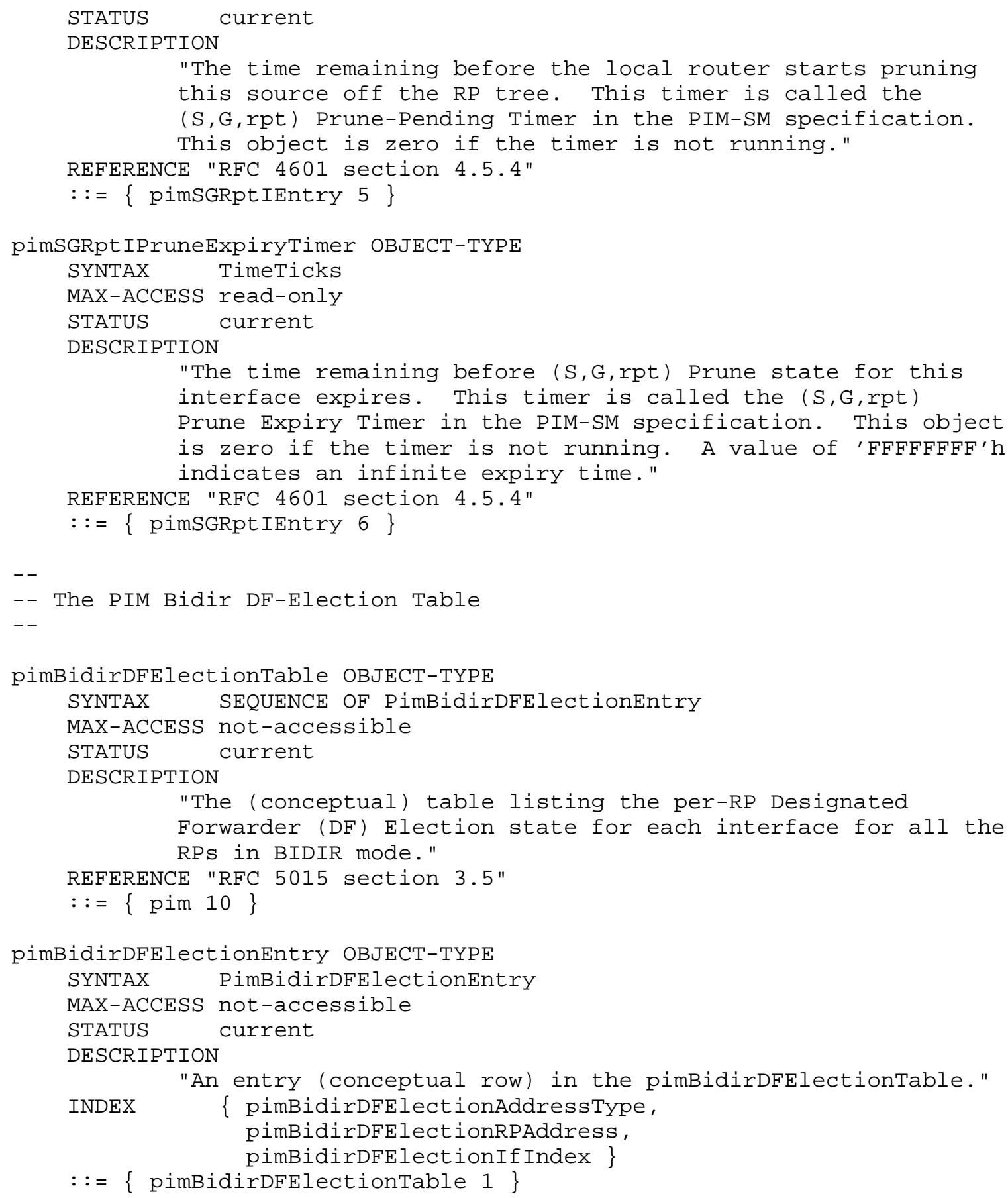




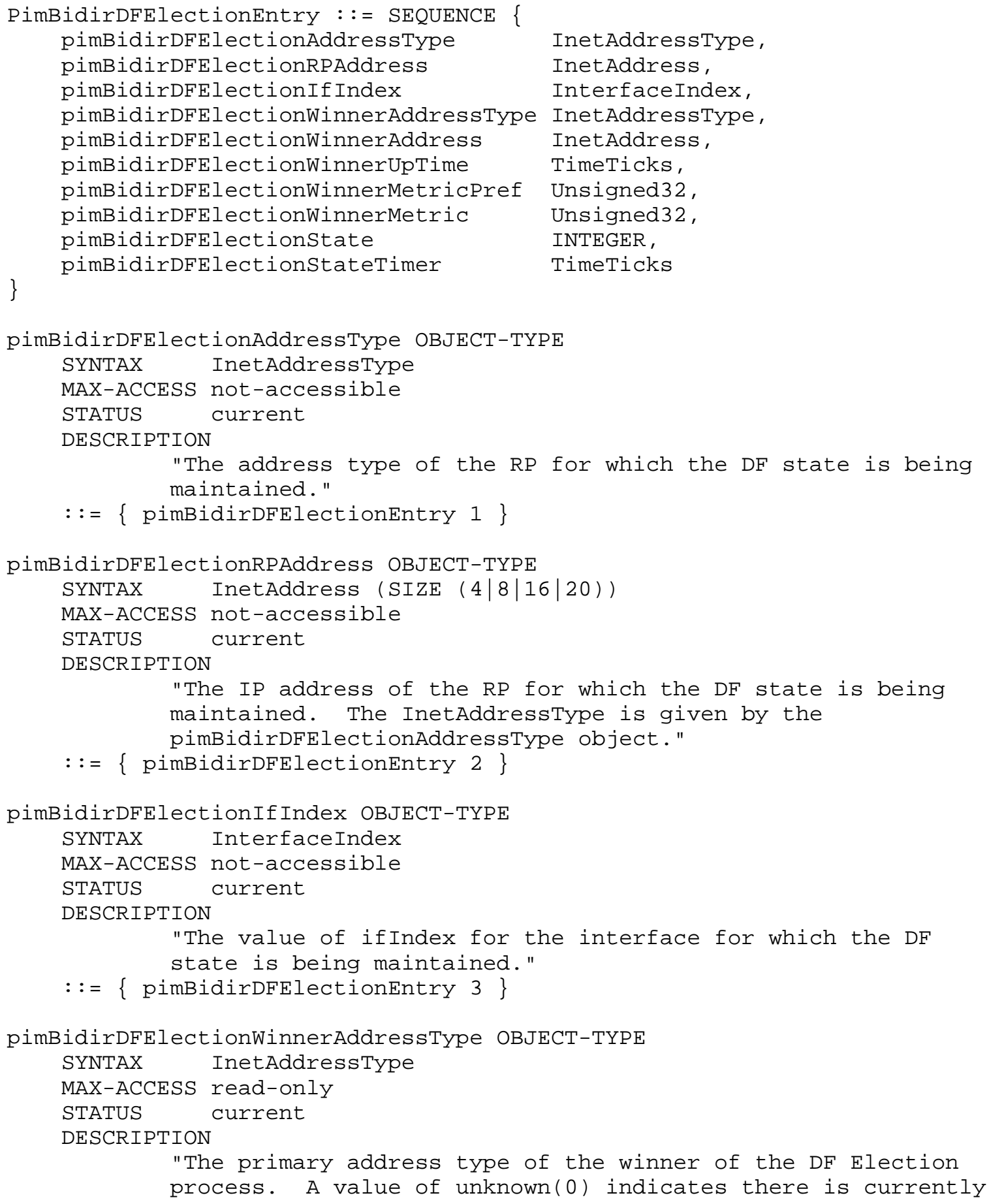




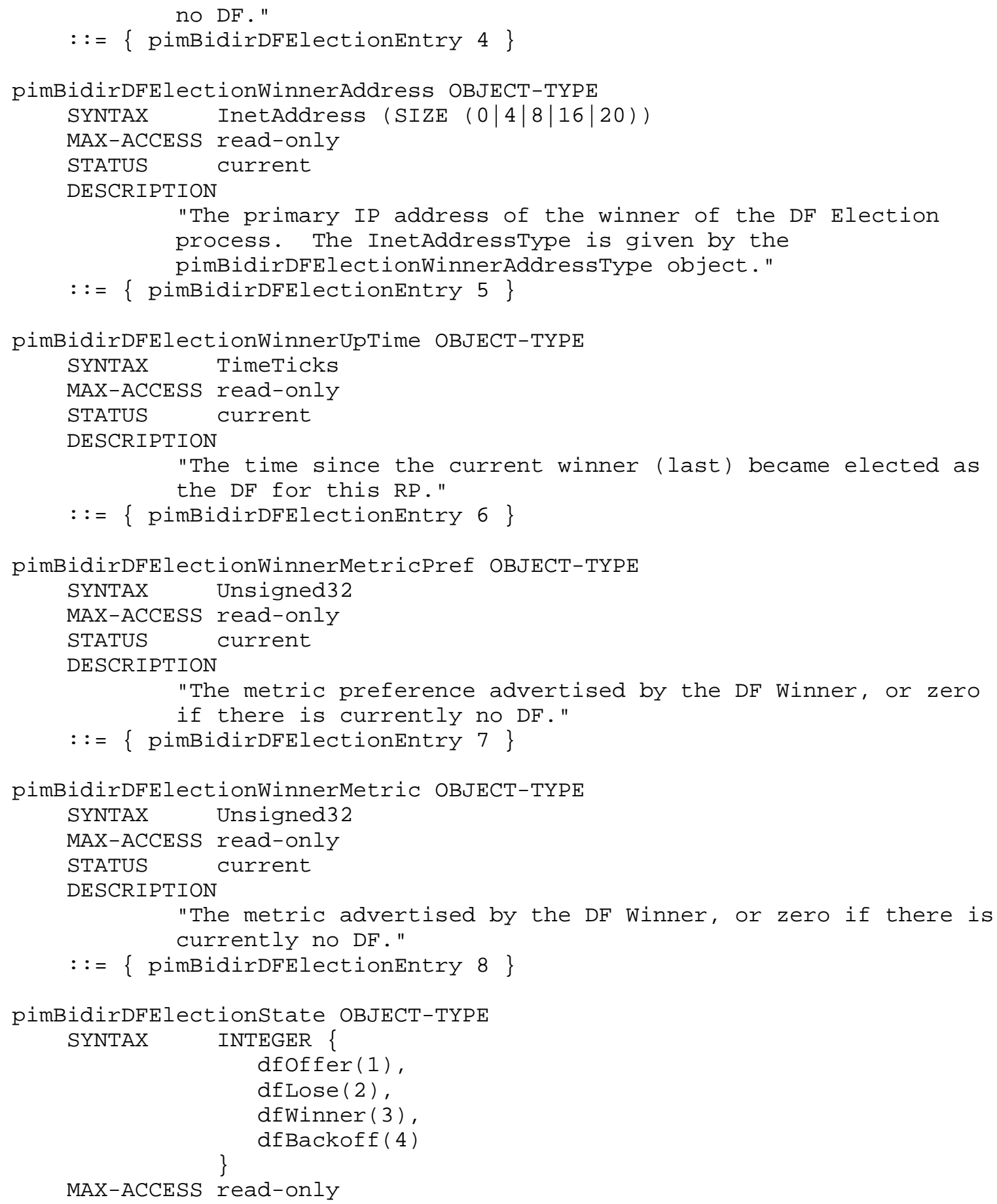




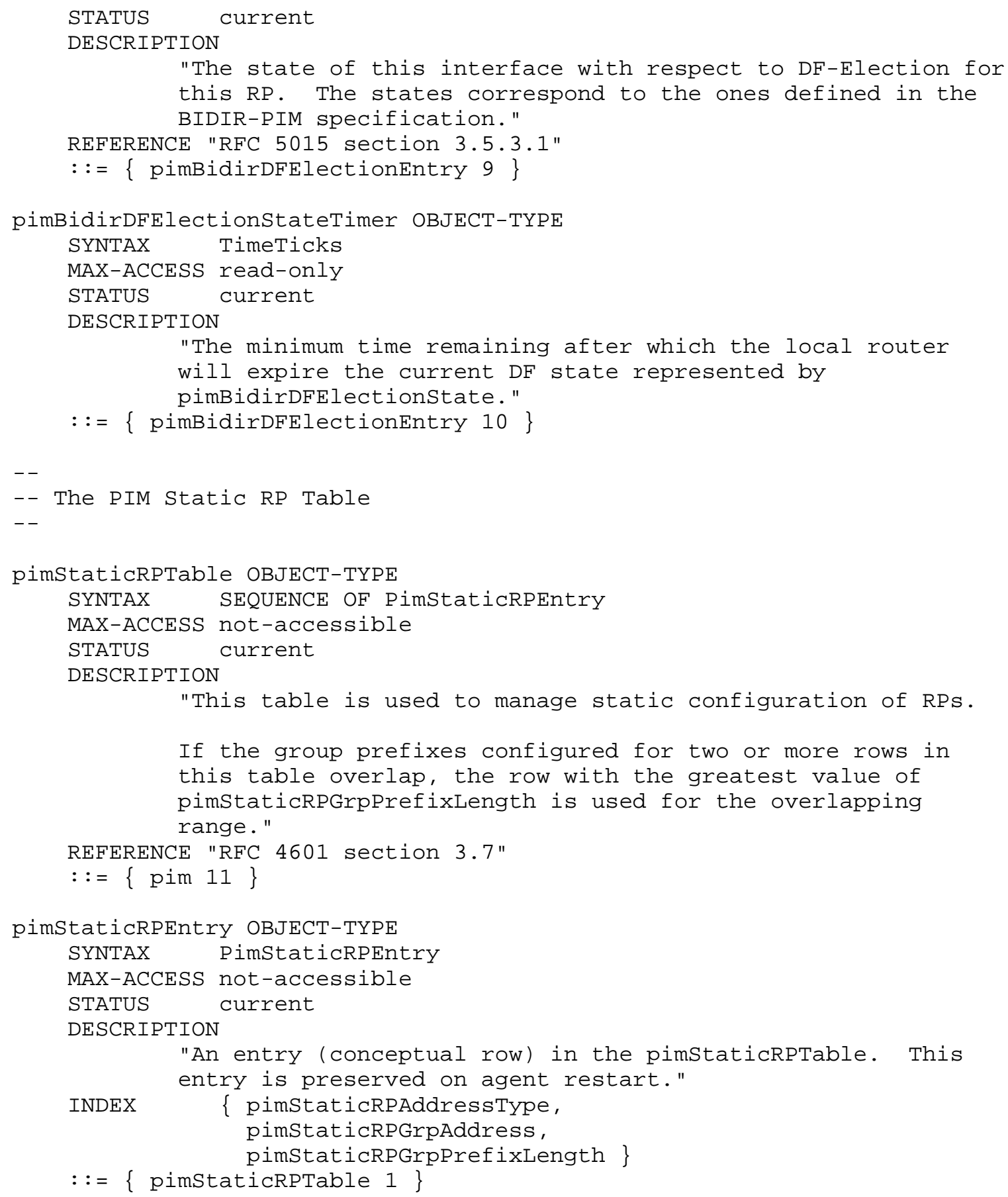




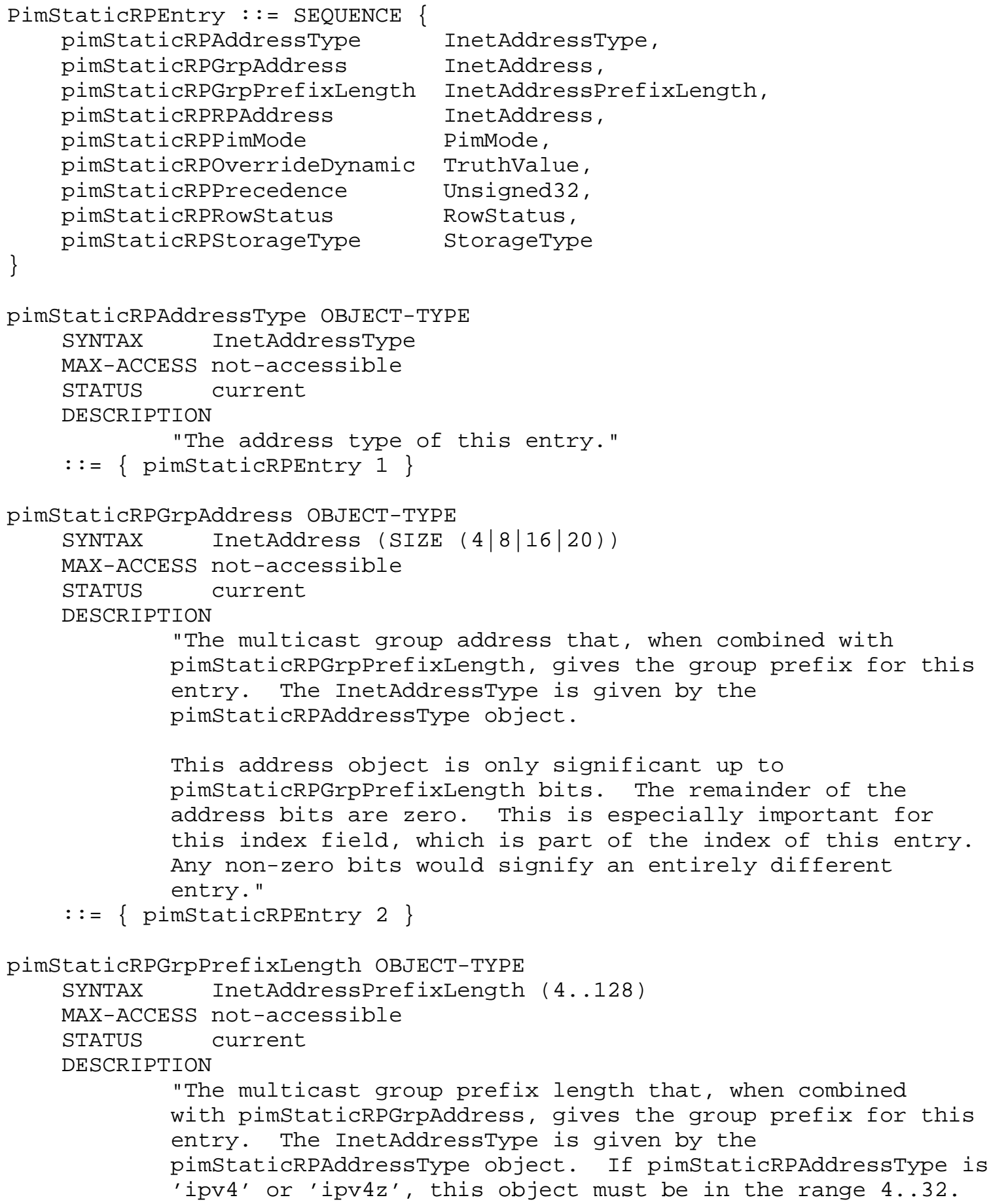




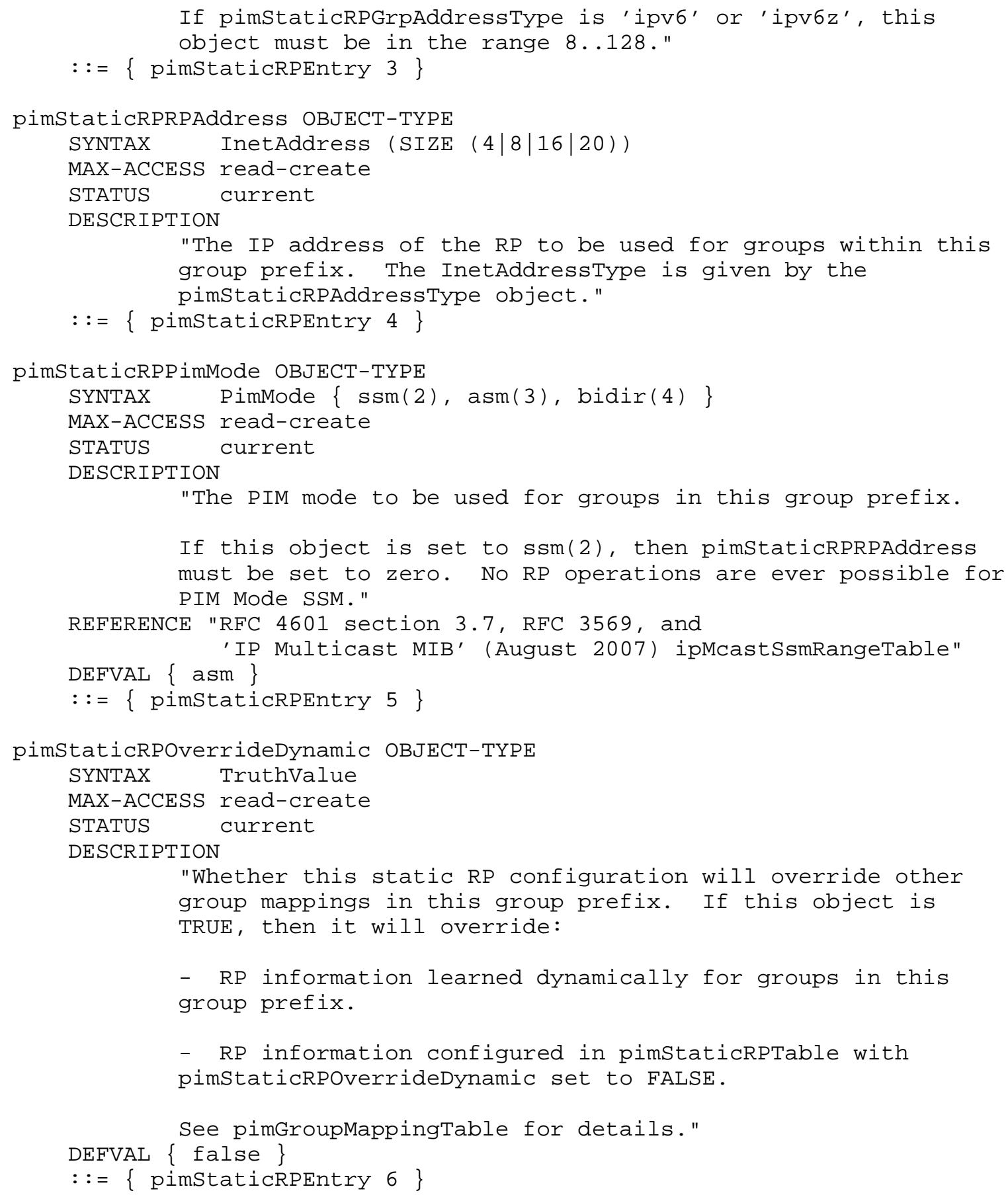




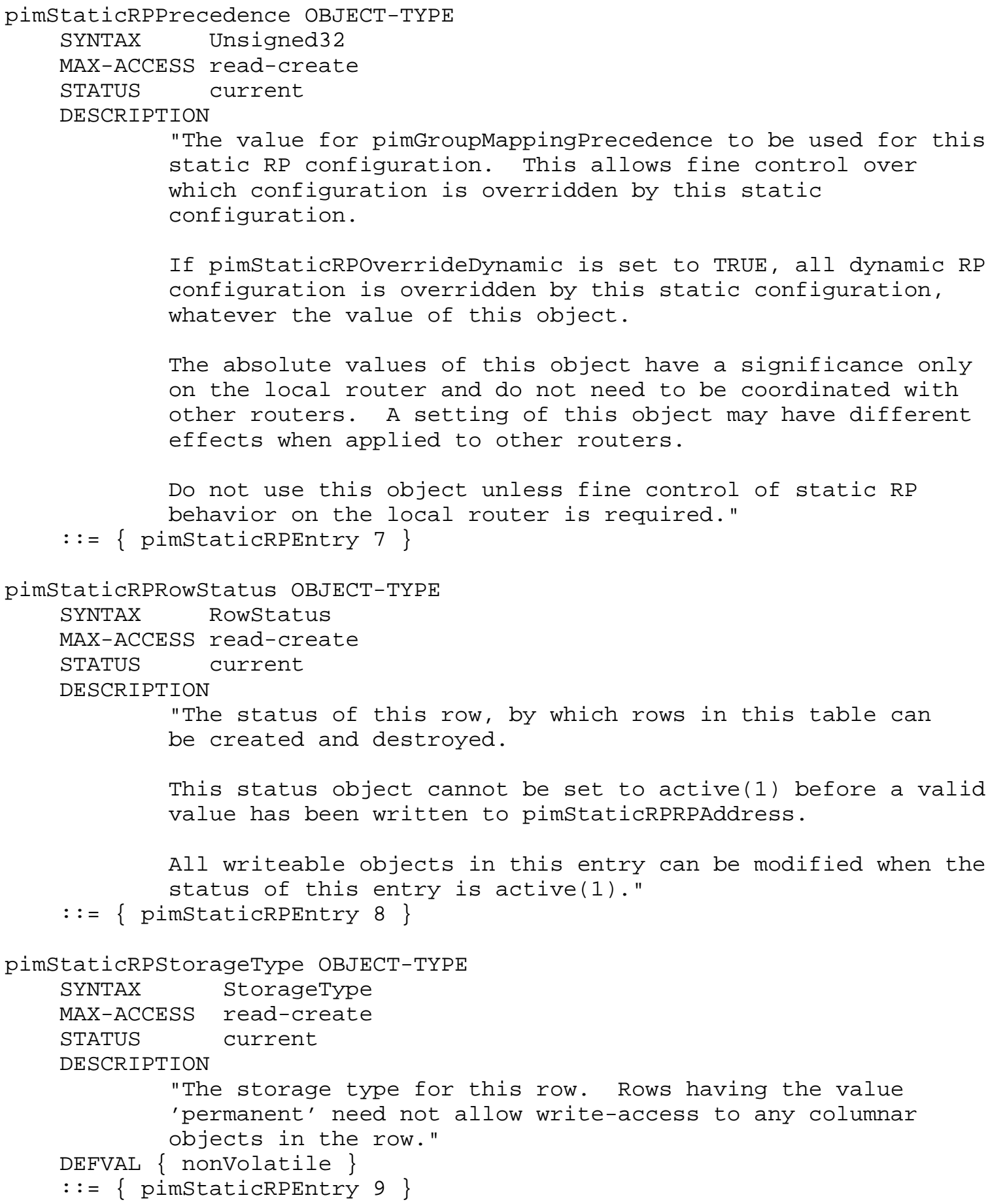




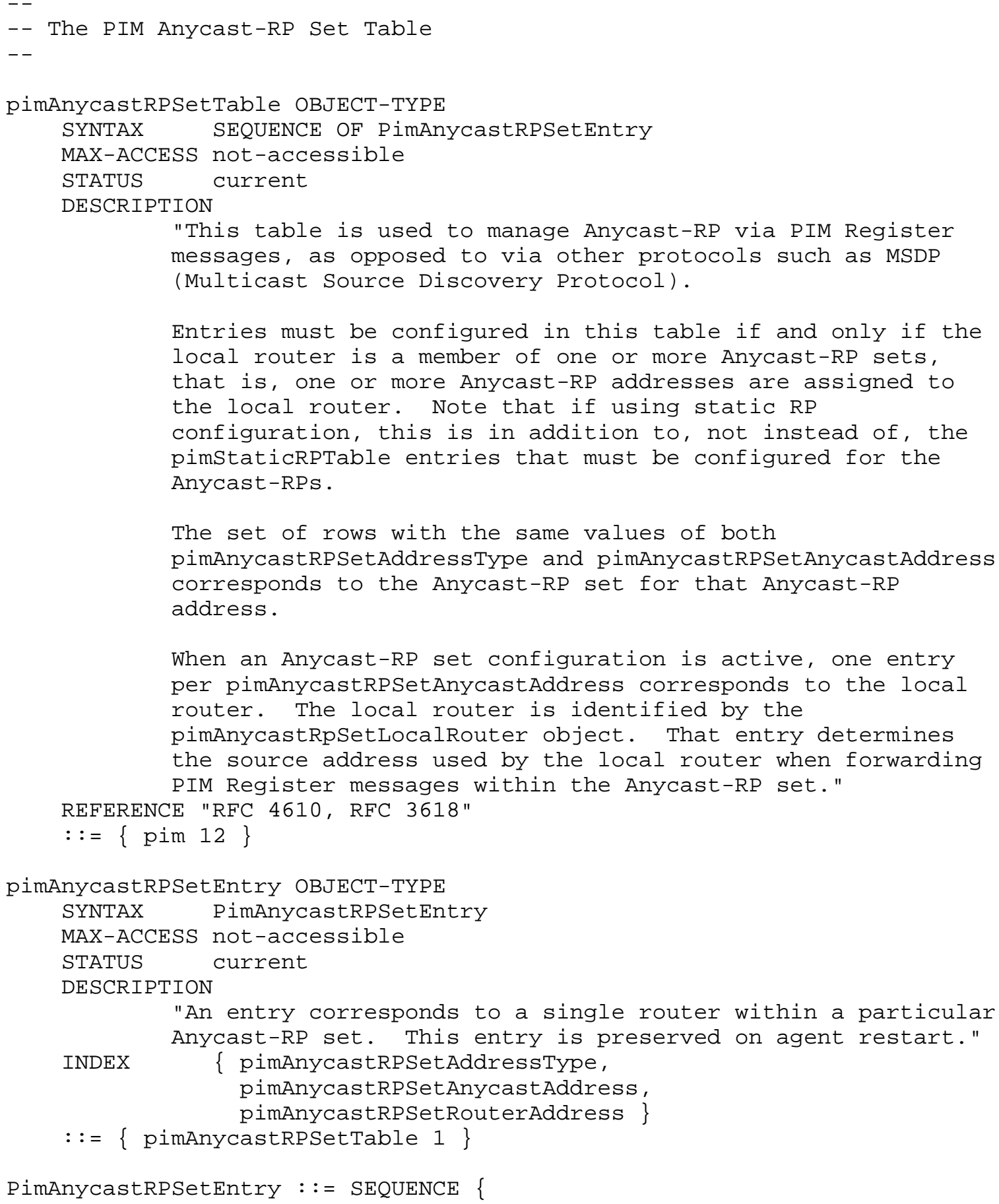




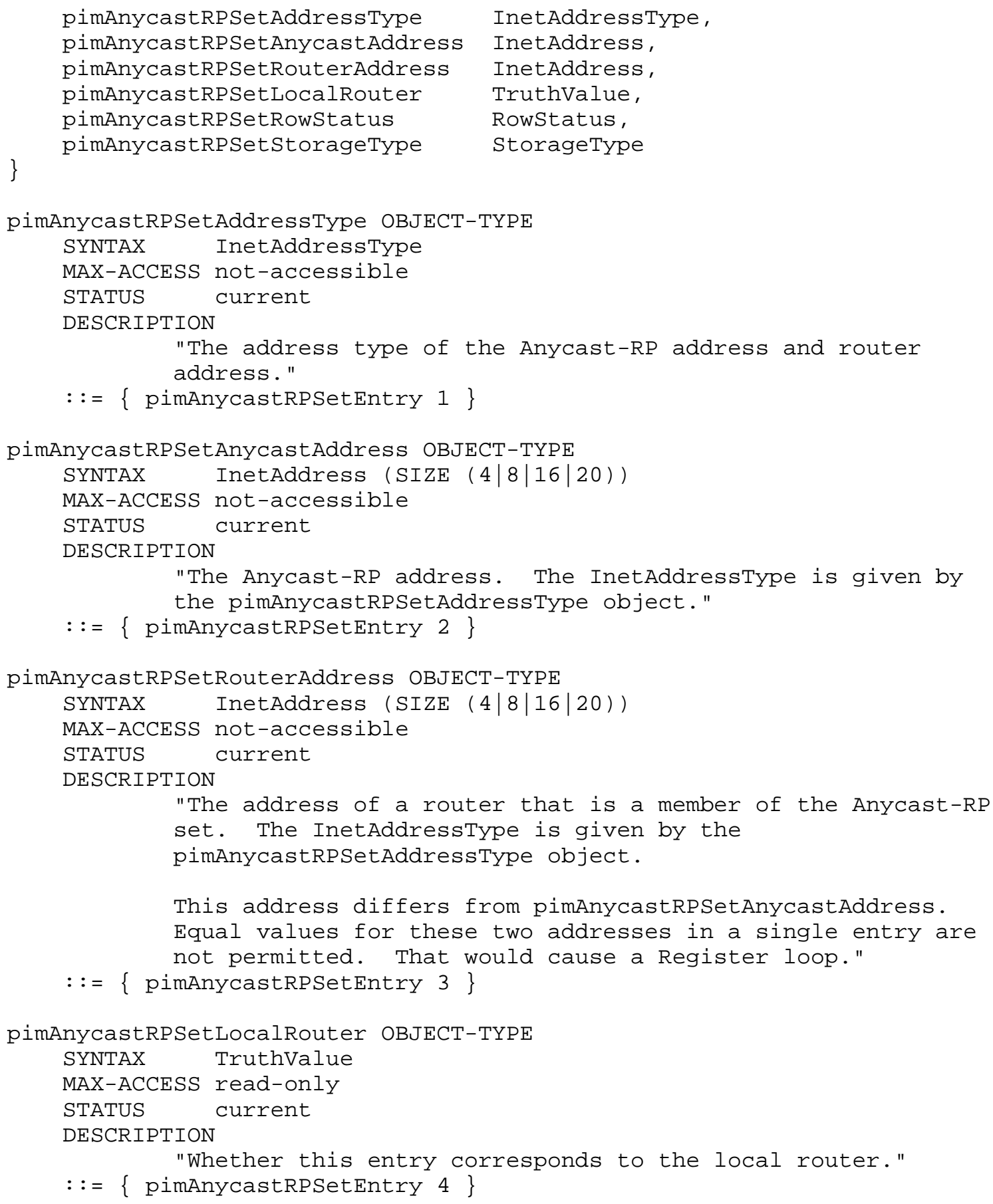




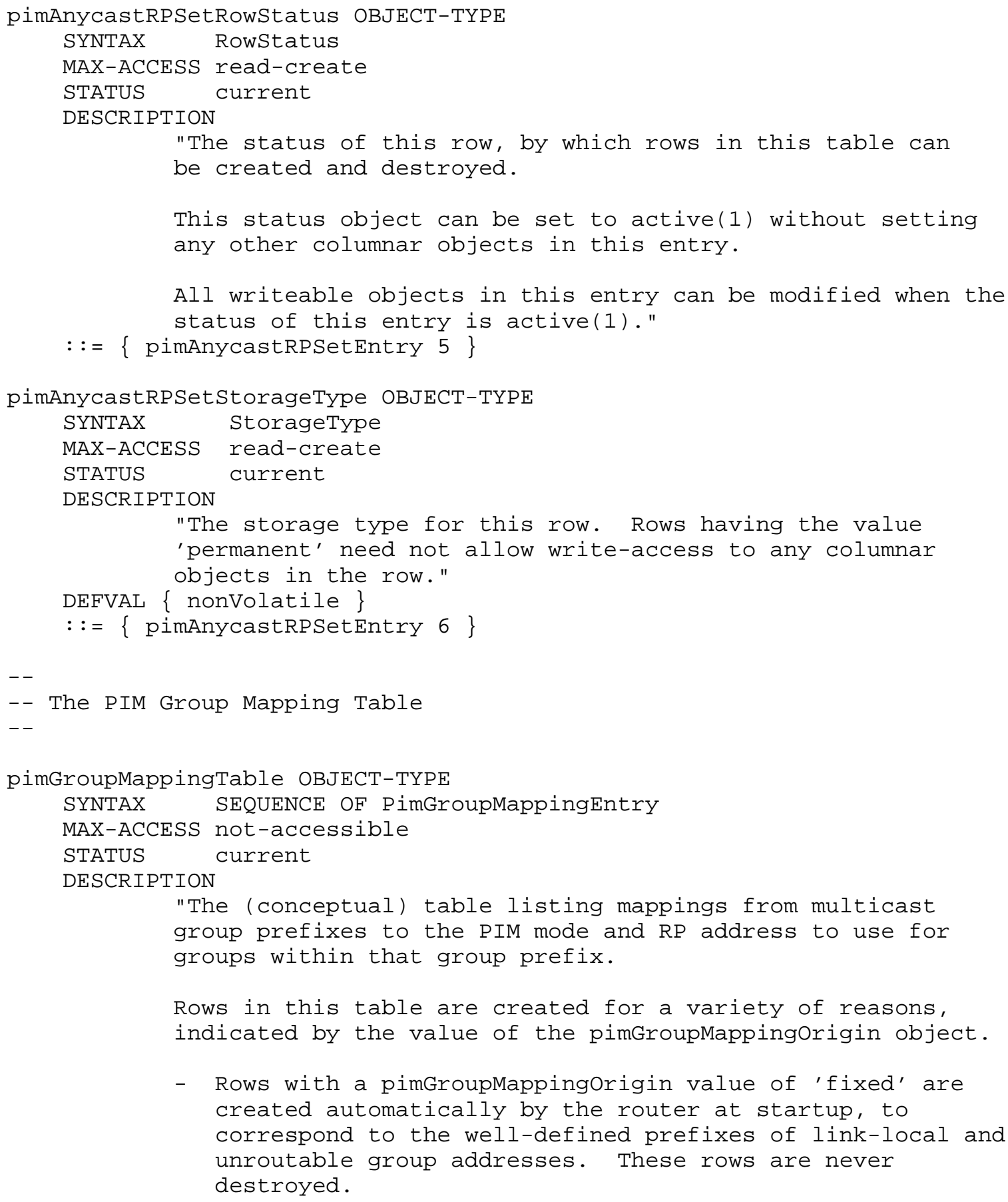


- Rows with a pimGroupMappingorigin value of 'embedded' are created by the router to correspond to group prefixes that are to be treated as being in Embedded-RP format.

- Rows with a pimGroupMappingorigin value of 'configRp' are created and destroyed as a result of rows in the pimstaticRPTable being created and destroyed.

- Rows with a pimGroupMappingOrigin value of 'configssm' are created and destroyed as a result of configuration of SSM address ranges to the local router.

- Rows with a pimGroupMappingorigin value of 'bsr' are created as a result of running the PIM Bootstrap Router (BSR) mechanism. If the local router is not the elected BSR, these rows are created to correspond to group prefixes in the PIM Bootstrap messages received from the elected BSR. If the local router is the elected BSR, these rows are created to correspond to group prefixes in the PIM Bootstrap messages that the local router sends. In either case, these rows are destroyed when the group prefixes are timed out by the BSR mechanism.

- Rows with a pimGroupMappingorigin value of 'other' are created and destroyed according to some other mechanism not specified here.

Given the collection of rows in this table at any point in time, the PIM mode and RP address to use for a particular group is determined using the following algorithm.

1. From the set of all rows, the subset whose group prefix contains the group in question are selected.

2. If there are no such rows, then the group mapping is undefined.

3. If there are multiple selected rows, and a subset is defined by pimstaticRPTable (pimGroupMappingorigin value of 'configRp') with pimstaticRPOverrideDynamic set to TRUE, then this subset is selected.

4. From the selected subset of rows, the subset that have the greatest value of pimGroupMappingGrpPrefixLength are selected.

5. If there are still multiple selected rows, the subset that has the highest precedence (the lowest numerical 
value for pimGroupMappingPrecedence) is selected.

6. If there are still multiple selected rows, the row selected is implementation dependent; the implementation might or might not apply the PIM hash function to select the row.

7. The group mode to use is given by the value of pimGroupMappingPimMode from the single selected row; the $\mathrm{RP}$ to use is given by the value of pimGroupMappingRPAddress, unless pimGroupMappingorigin is ' embedded', in which case, the RP is extracted from the group address in question."

REFERENCE "RFC 4601 section 3.7, RFC 3956, and RFC 4610" $::=\{\operatorname{pim} 13\}$

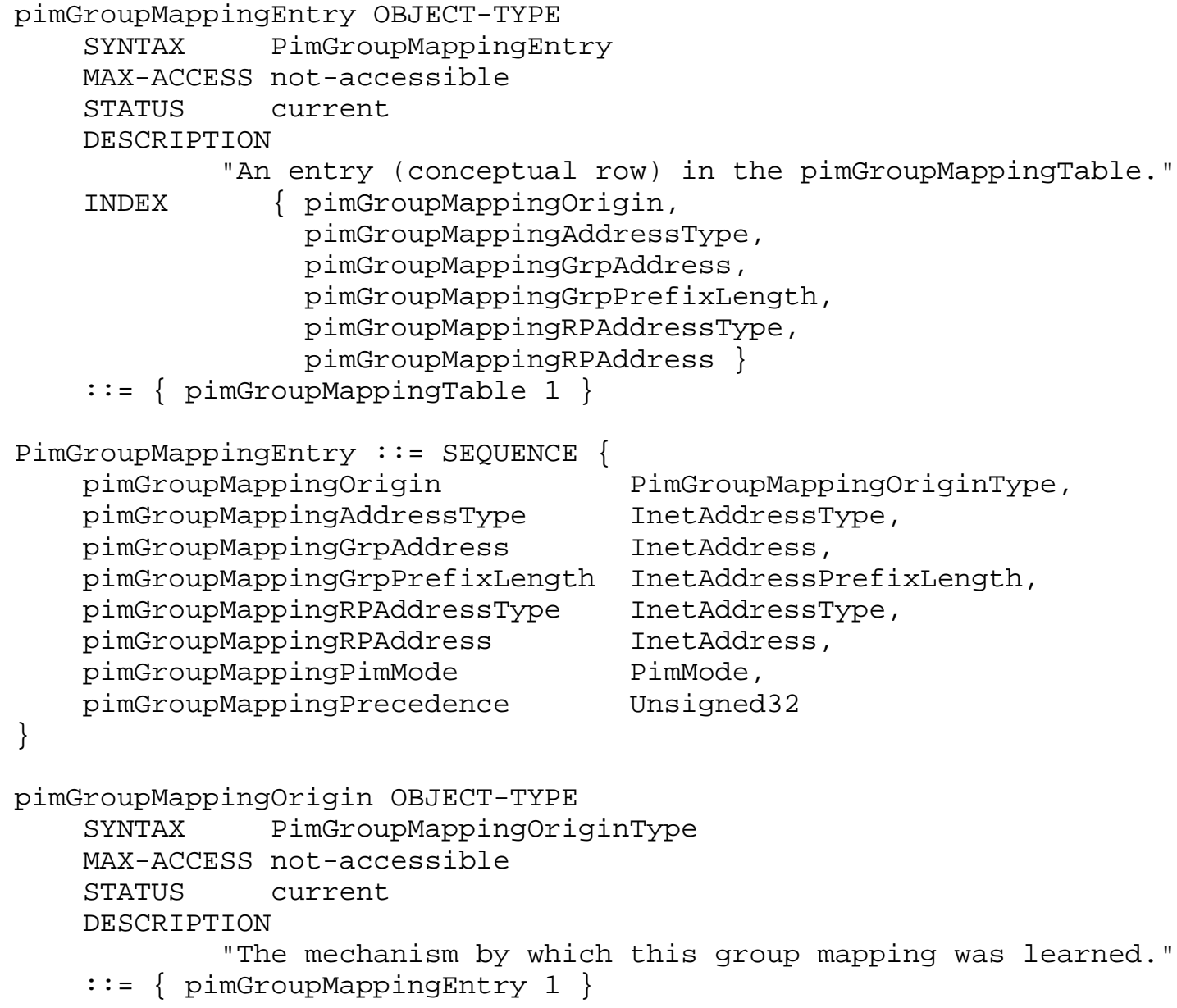




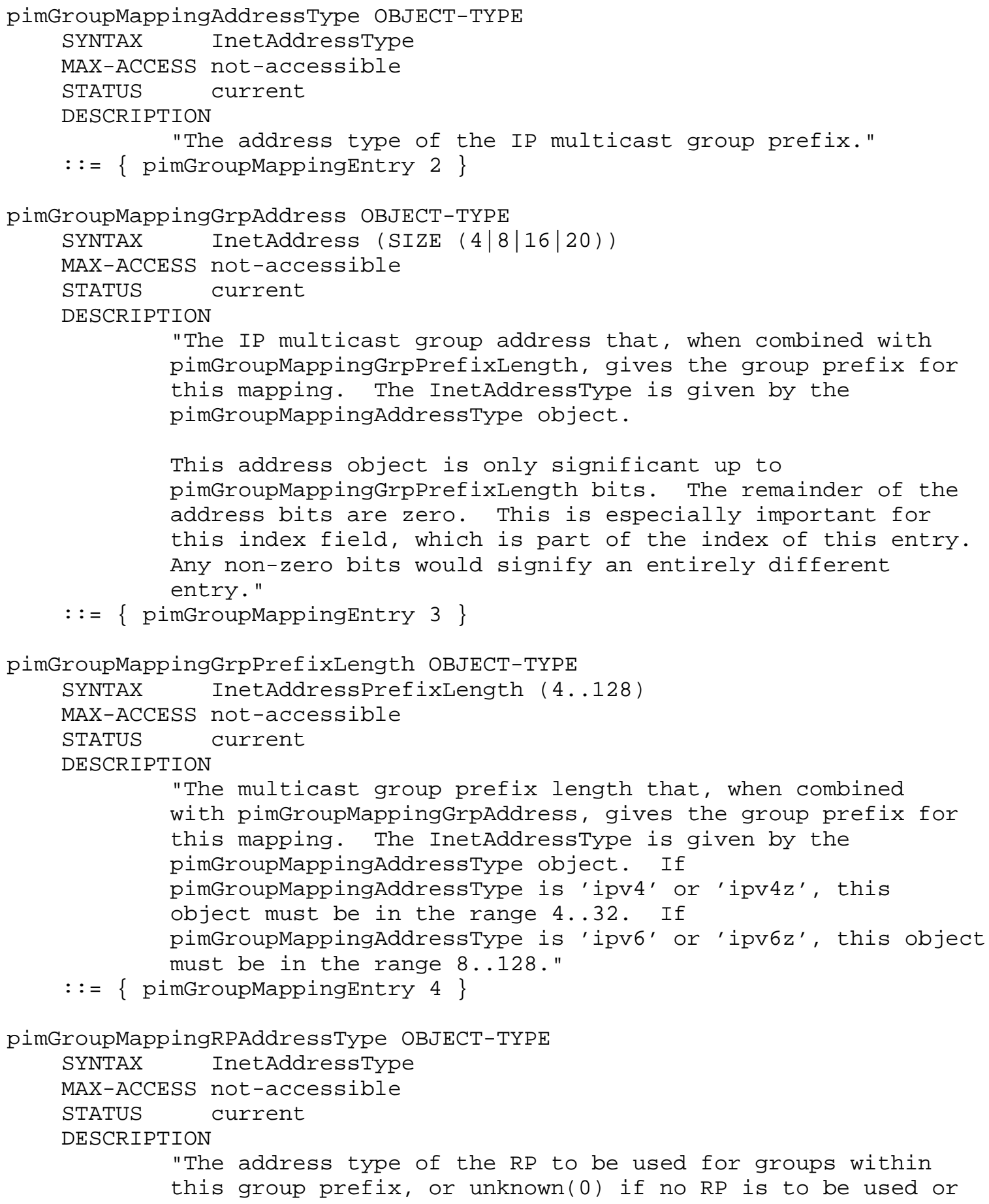




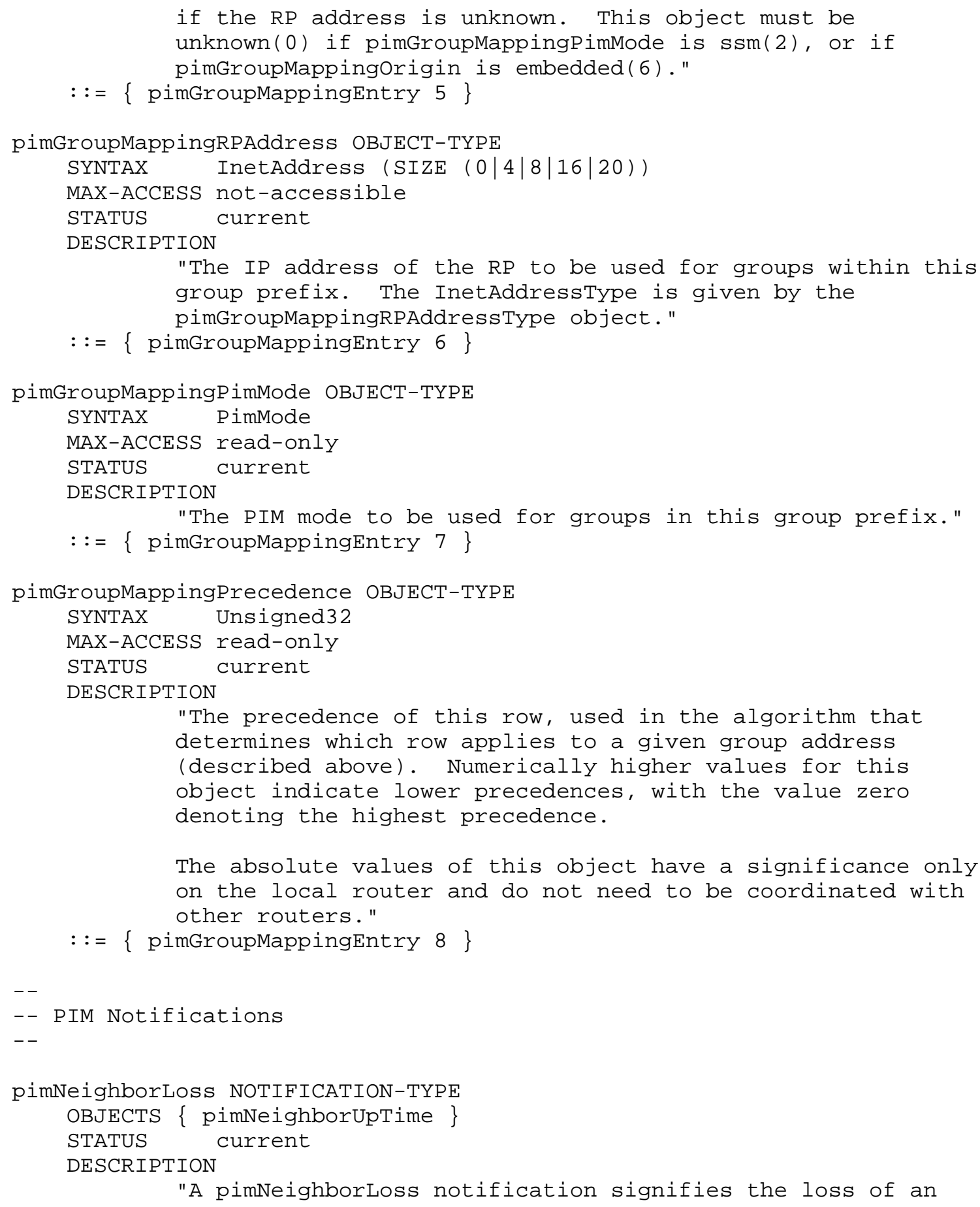




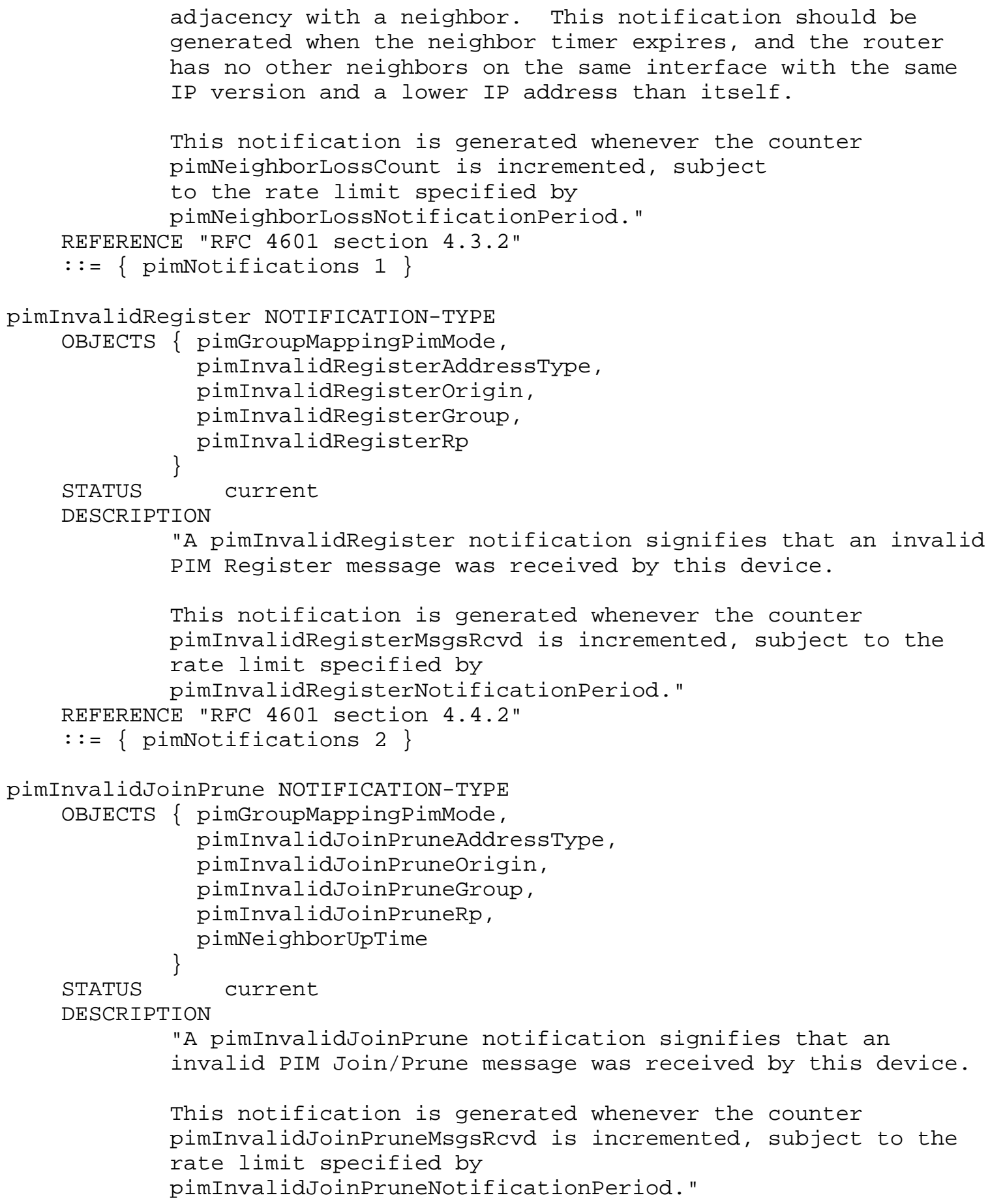




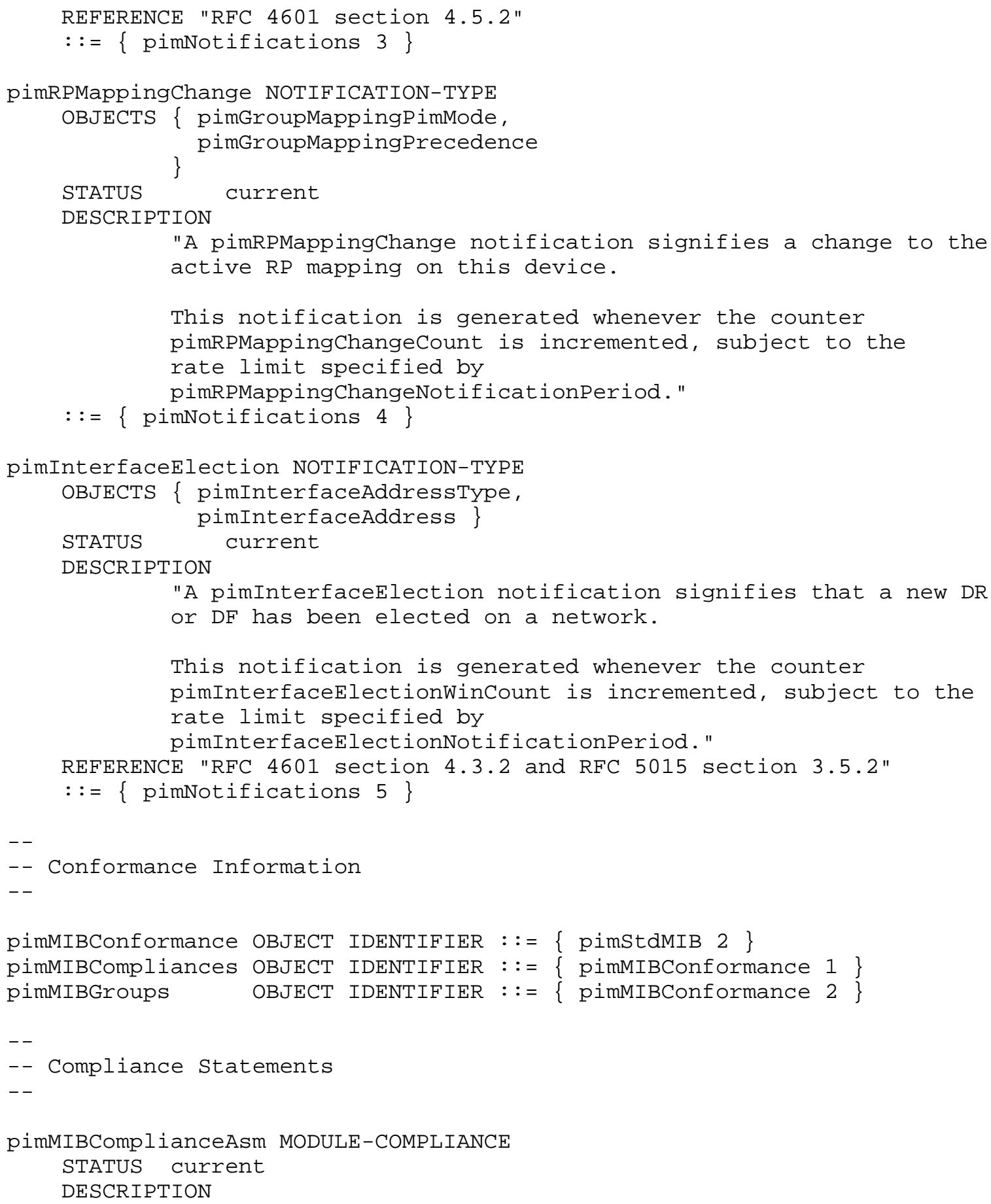




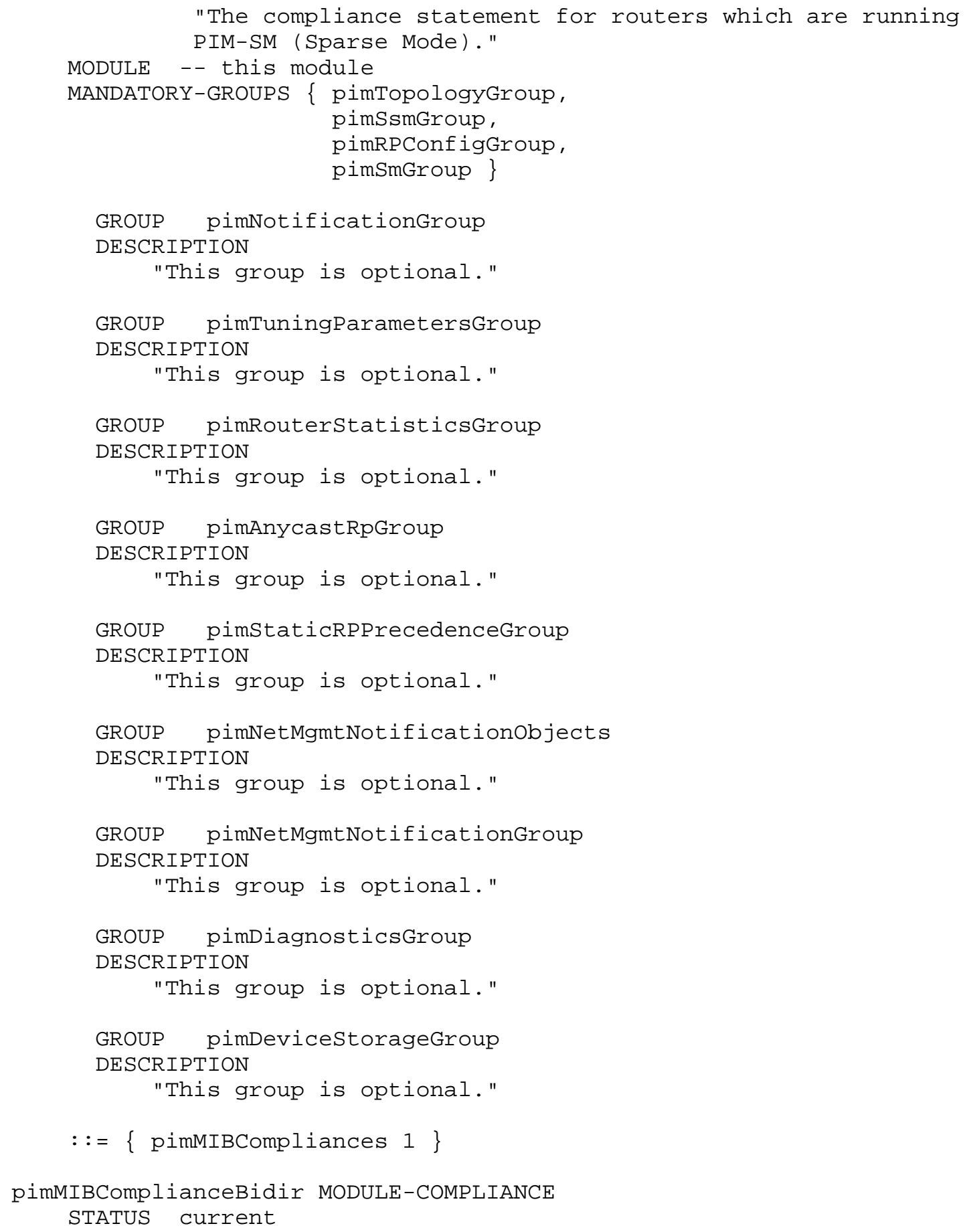




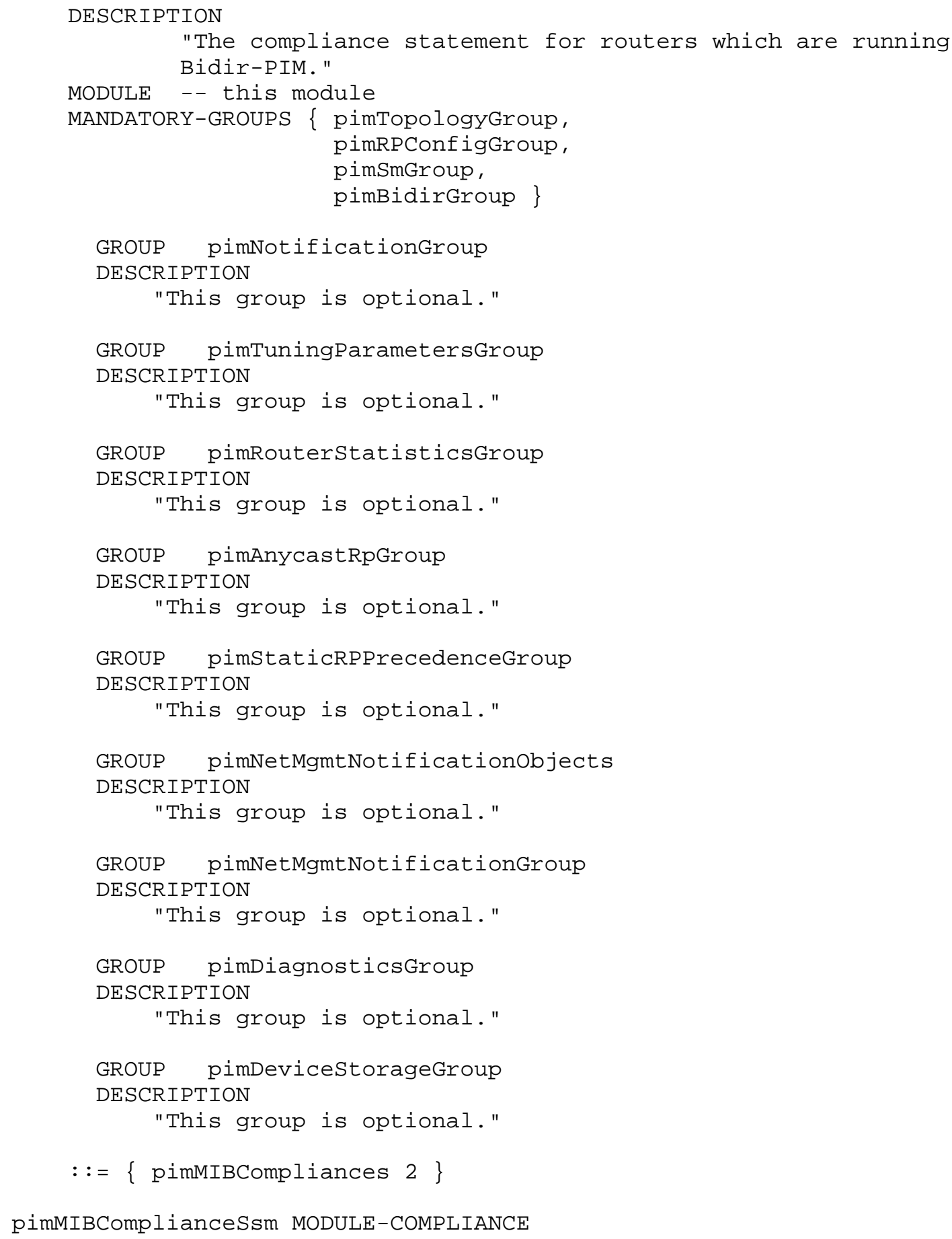




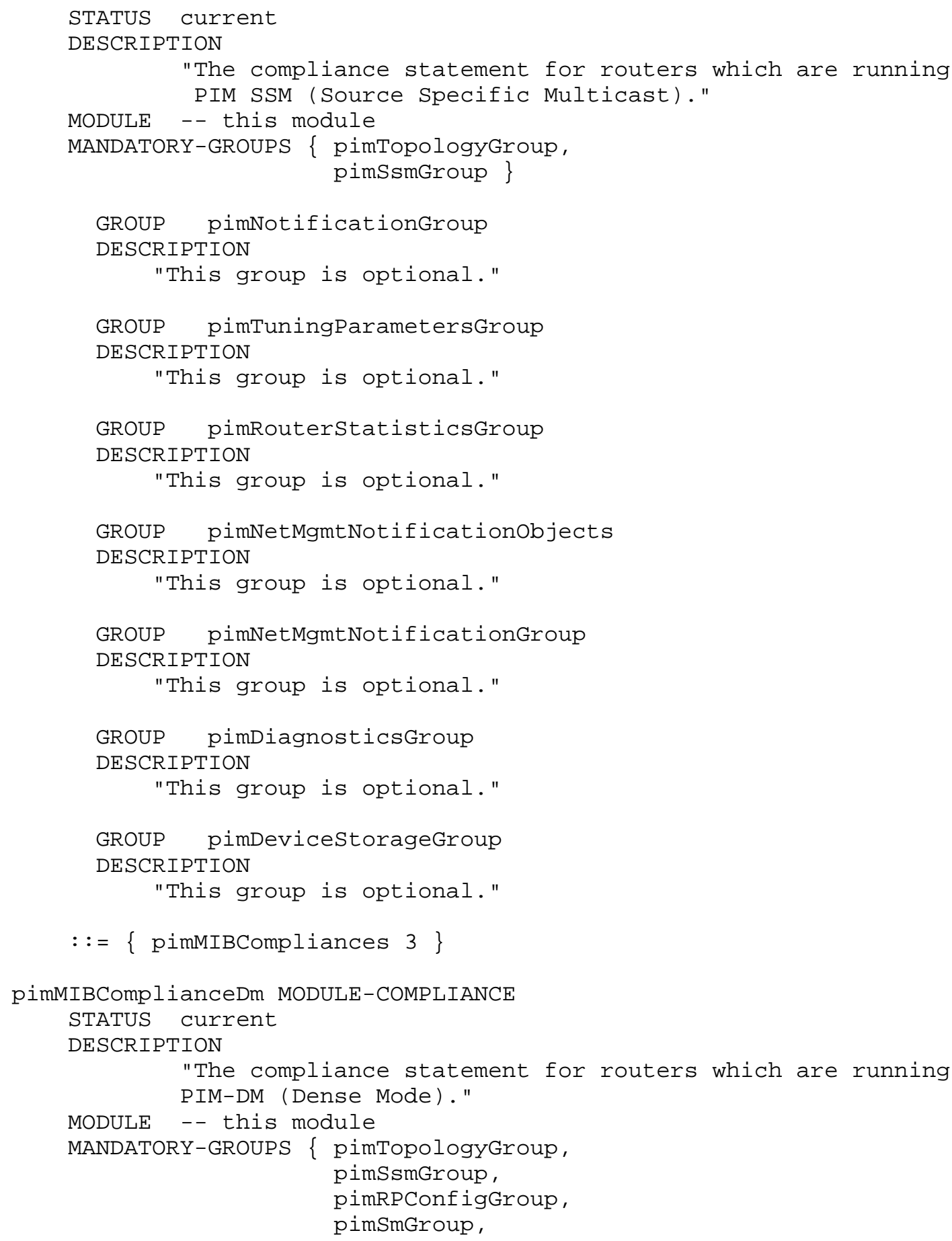




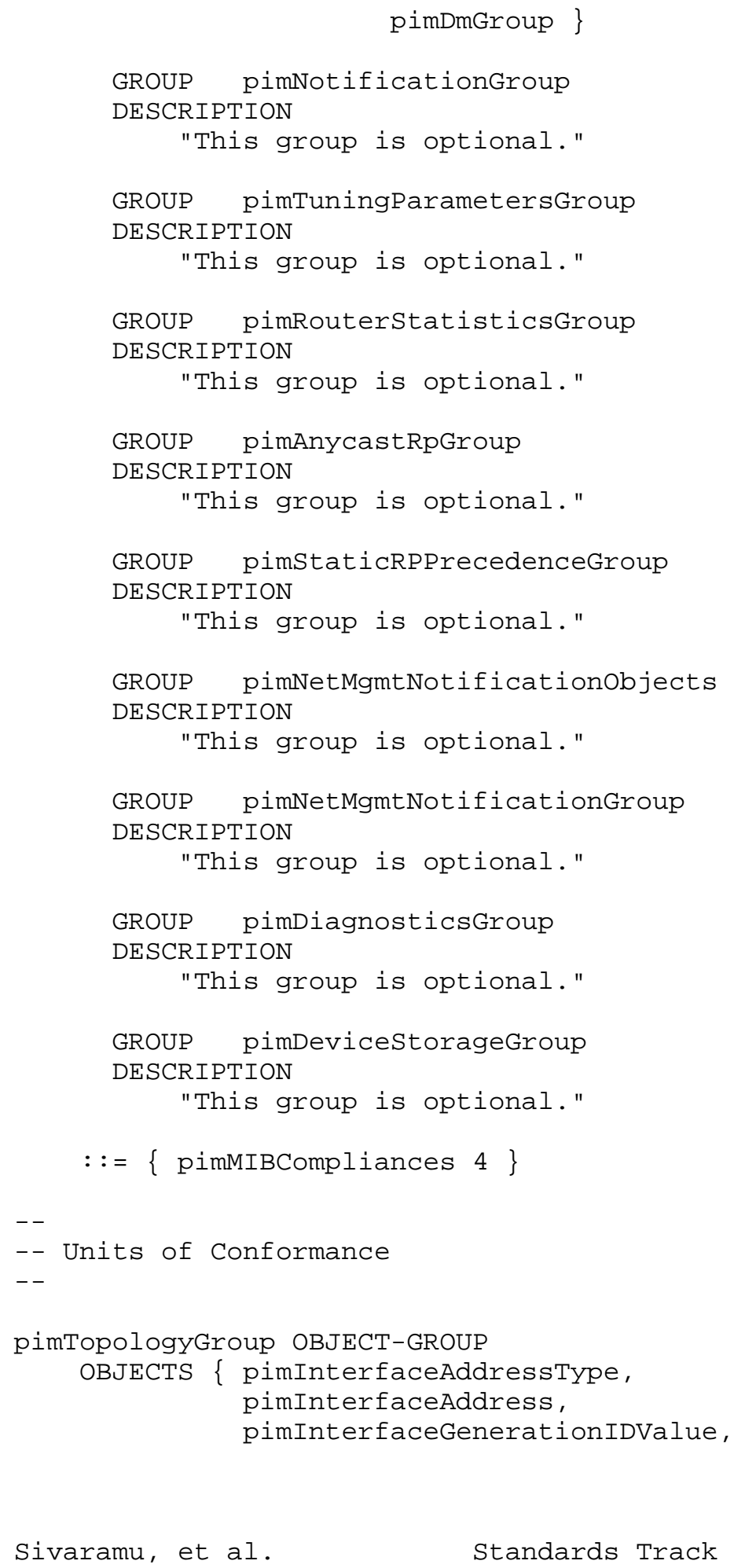




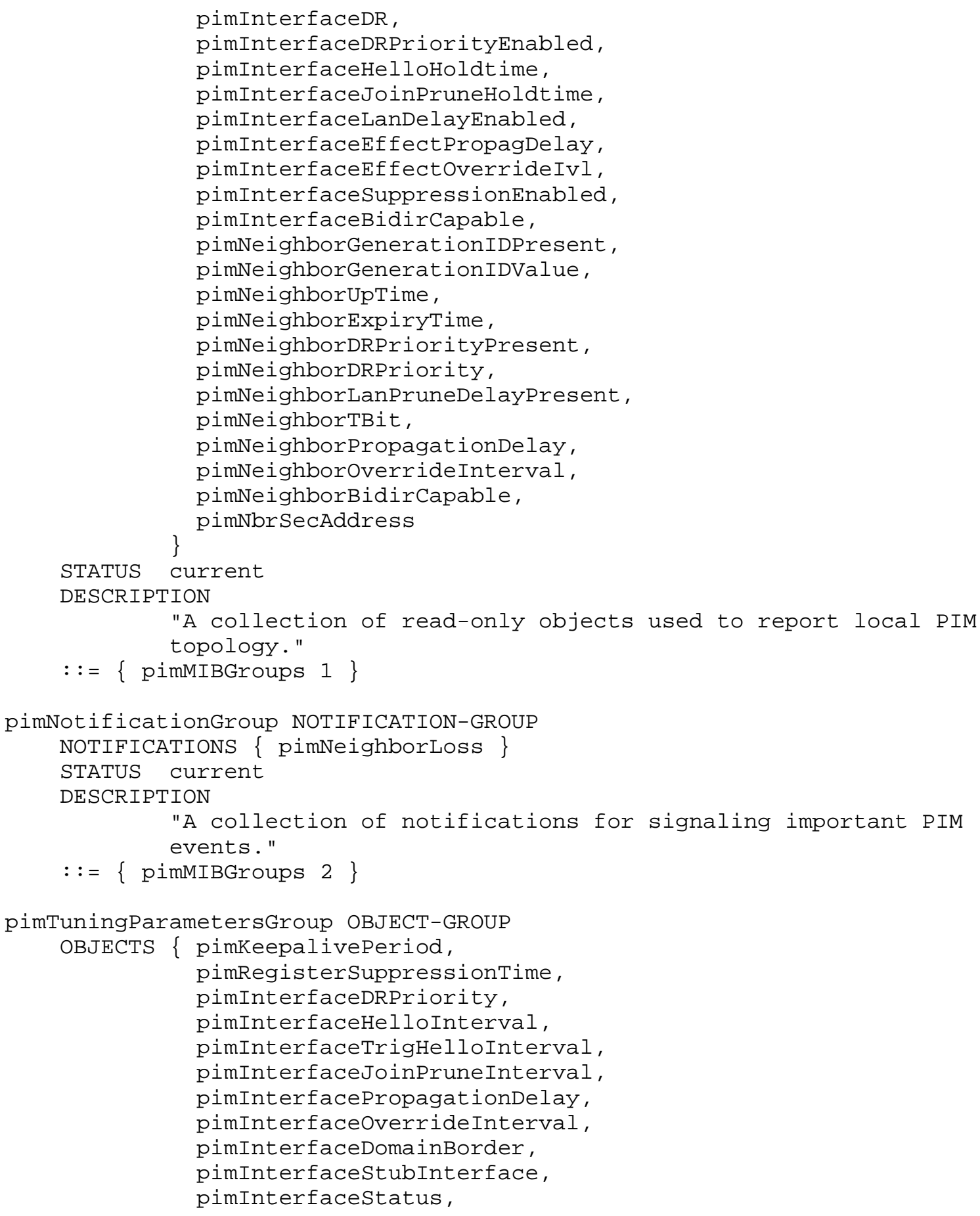




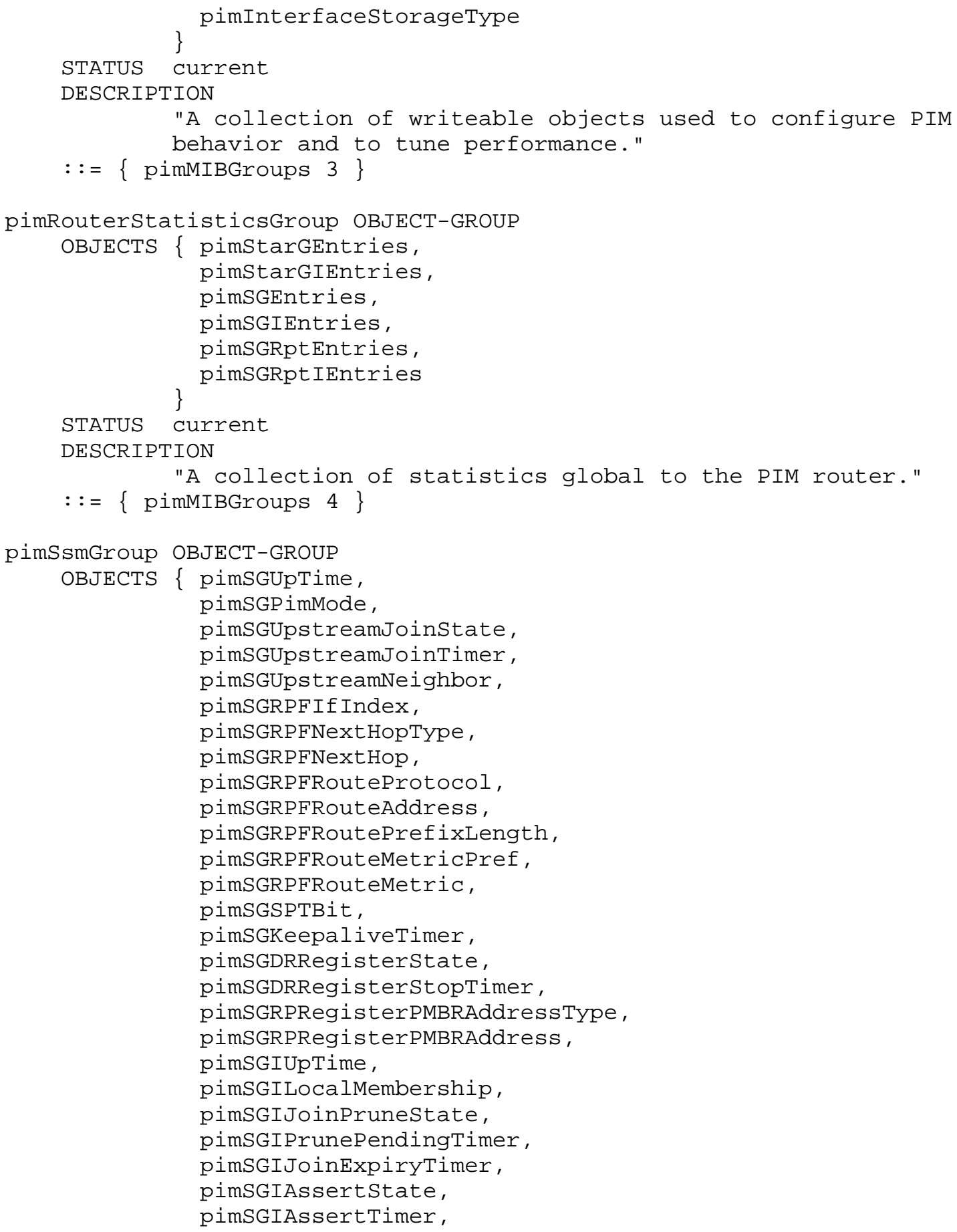




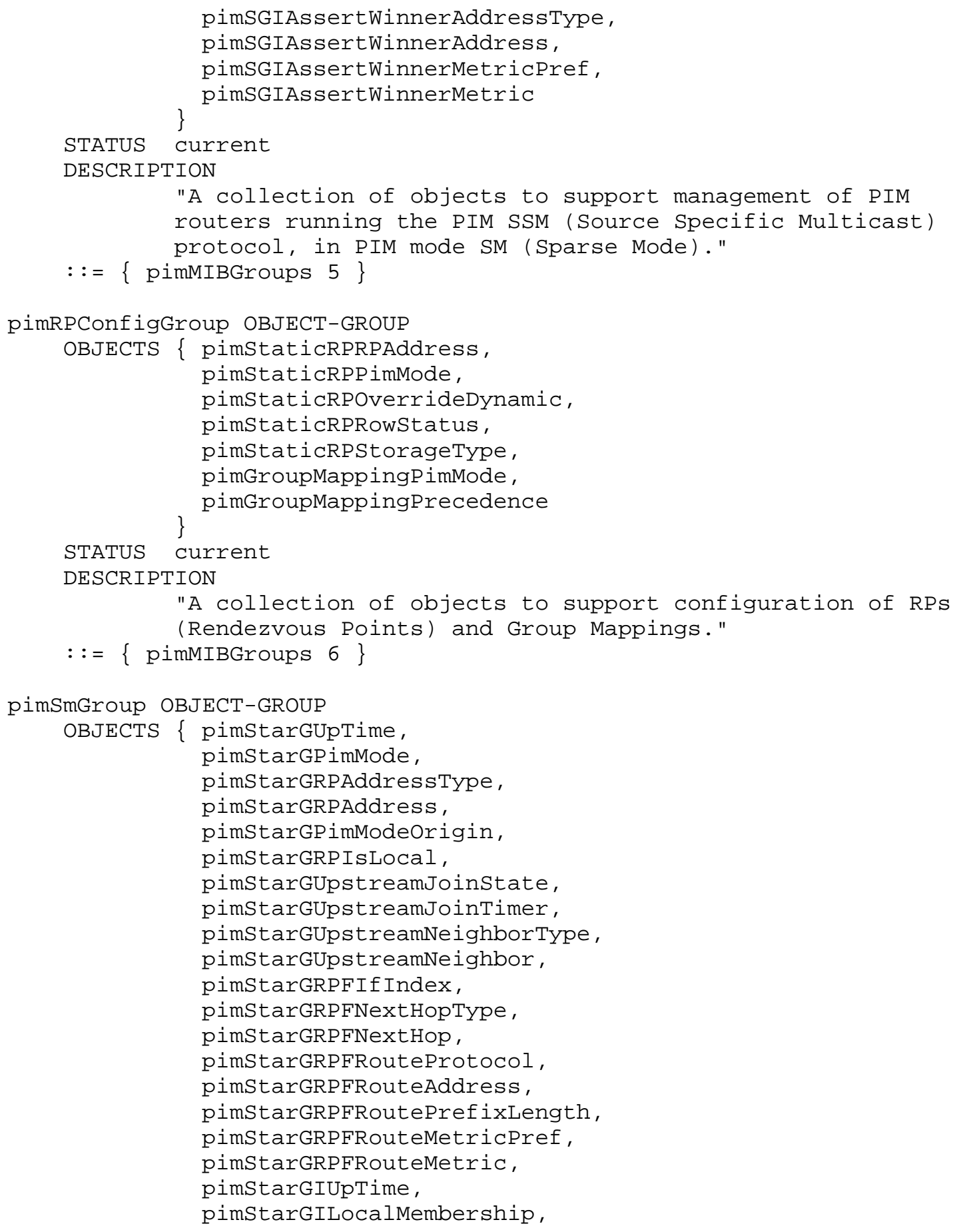




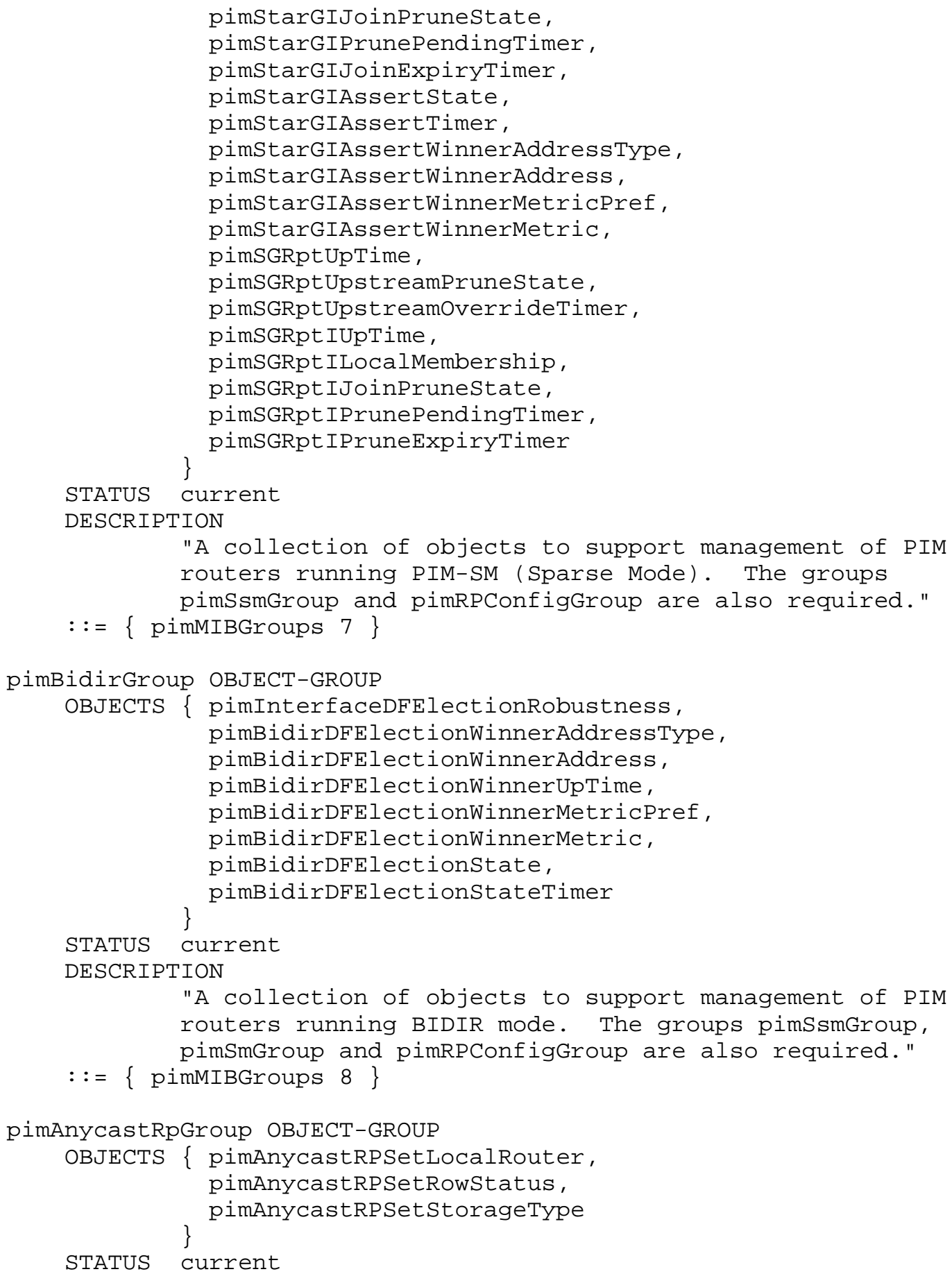




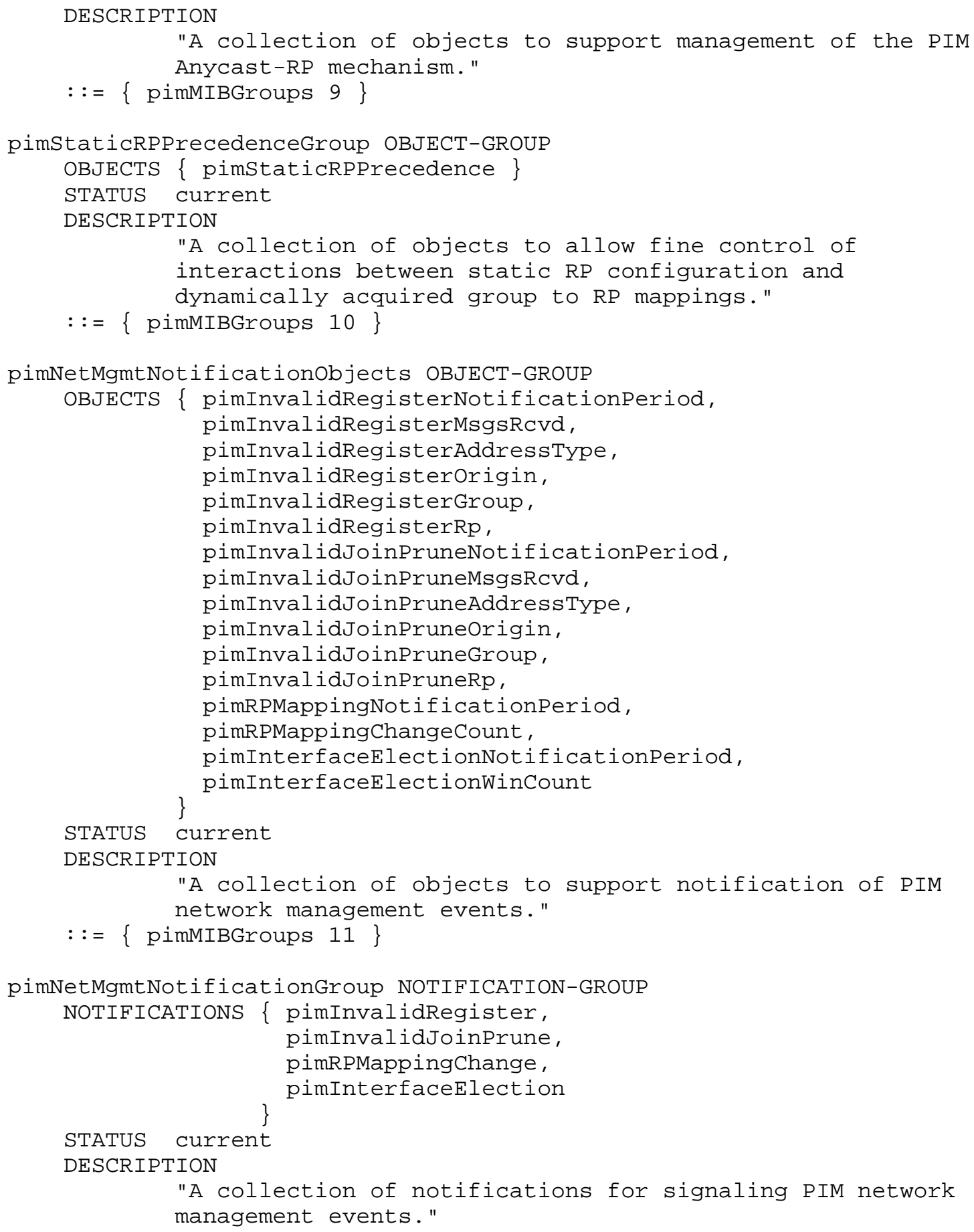




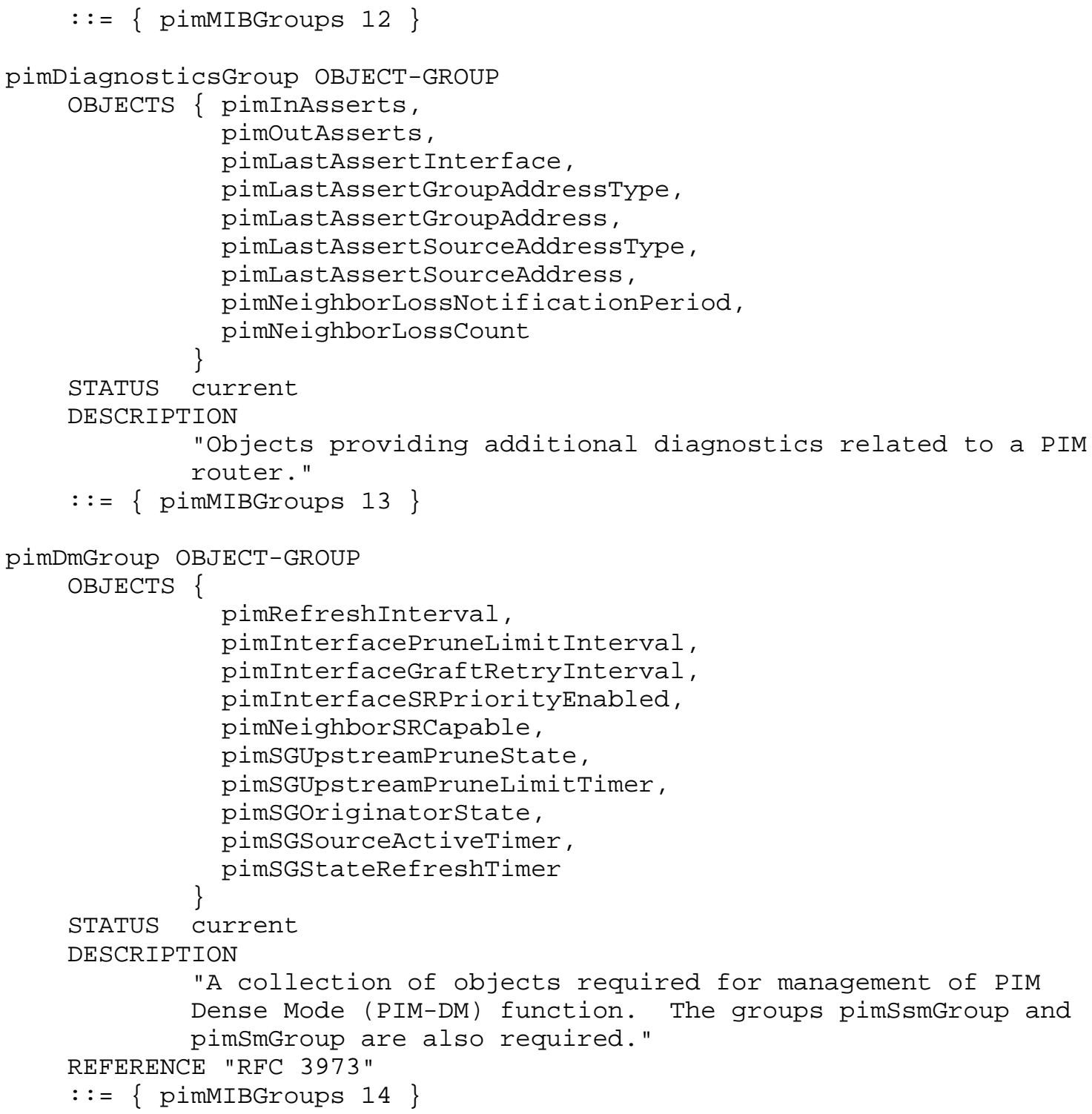




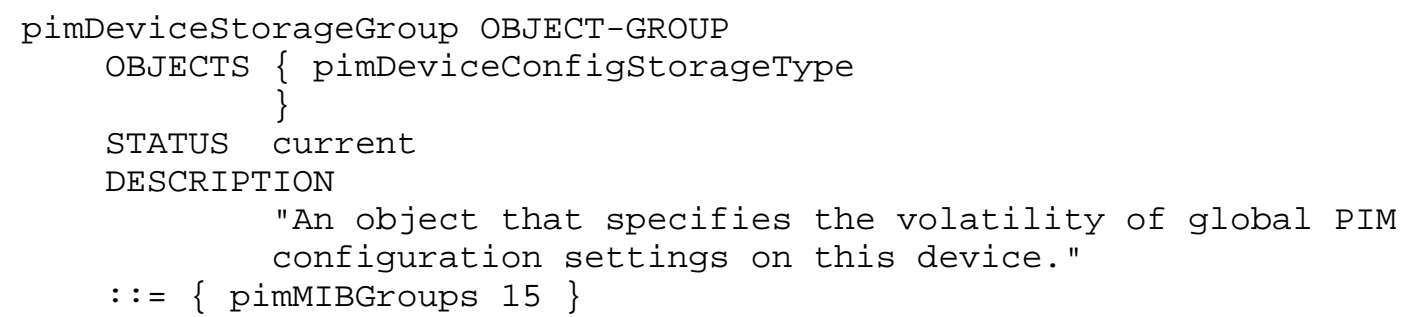

END

6. Security Considerations

There are a number of management objects defined in this MIB module with a MAX-ACCESS clause of read-write and/or read-create. Such objects may be considered sensitive or vulnerable in some network environments. The support for SET operations in a non-secure environment without proper protection can have a negative effect on network operations. These are the tables and objects and their sensitivity/vulnerability:

The following tables and objects could be employed to modify multicast routing behavior in a way that prevents, disrupts, or subverts services provided by the network, including (but not limited to) multicast data traffic delivery. For example, attacks can be envisaged that would pass nominated multicast data streams through a nominated location, without the sources or listeners becoming aware of this subversion.

pimKeepalivePeriod pimRegisterSuppressionTime

pimNeighborLossNotificationPeriod

pimInvalidRegisterNotificationPeriod

pimInvalidJoinPruneNotificationPeriod pimRPMappingNotificationPeriod pimInterfaceElectionNotificationPeriod pimRefreshInterval pimInterfaceTable pimInterfaceEntry pimInterfaceIfIndex pimInterfaceIPVersion pimInterfaceHelloInterval pimInterfaceTrigHelloInterval pimInterfaceJoinPruneInterval pimInterfaceDFElectionRobustness pimInterfaceHelloHoldtime pimInterfaceJoinPruneHoldtime pimInterfacePropagationDelay pimInterfaceOverrideInterval pimInterfaceDRPriority pimInterfaceDomainBorder pimInterfacestatus pimInterfacestubInterface pimInterfacePruneLimitInterval pimStaticRPTable pimstaticRPEntry pimstaticRPAddress Type pimstaticRPGrpAddress pimstaticRPGrpPrefixLength pimstaticRPRPAddress pimstaticRPPimMode pimstaticRPOverrideDynamic pimstaticRPRowstatus pimstaticRPPrecedence pimAnycastRPSetTable pimAnycastRPSetEntry pimAnycastRPSetAddressType pimAnycastRPSetAnycastAddress pimAnycastRPSetRouterAddress 
Some of the readable objects in this MIB module (i.e., objects with a MAX-ACCESS other than not-accessible) may be considered sensitive or vulnerable in some network environments. It is thus important to control even GET and/or NOTIFY access to these objects and possibly to even encrypt the values of these objects when sending them over the network via SNMP. These are the tables and objects and their sensitivity/vulnerability:

The following tables and objects could be employed to determine the topology, disposition, and composition of the network. This information may be commercially sensitive, and may also be used in preparation for attacks, including any of the attacks described above.

The following tables and objects may also be used to determine whether multicast data is flowing in the network, or has flowed recently. They may also be used to determine the network location of senders and recipients. An attacker can apply 'traffic analysis' to this data. In some cases, the information revealed by traffic analyses can be as damaging as full knowledge of the data being transported.

pimKeepalivePeriod pimRegisterSuppressionTime pimStarGEntries pimstarGIEntries pimSGEntries pimSGIEntries pimsGRptentries pimSGRptIEntries pimOutAsserts pimInAsserts pimLastAssertInterface pimLastAssertGroupAddressType pimLastAssertGroupAddress pimLastAssertSourceAddressType pimLastAssertSourceAddress pimNeighborLossNotificationPeriod pimNeighborLossCount pimInvalidRegisterNotificationPeriod pimInvalidRegisterMsgsRcvd pimInvalidRegisterAddressType pimInvalidRegisterOrigin pimInvalidRegisterGroup pimInvalidRegisterRp pimInvalidJoinPruneNotificationPeriod pimInvalidJoinPruneMsgsRcvd pimInvalidJoinPruneAddressType pimInvalidJoinPruneorigin pimInvalidJoinPruneGroup pimInvalidJoinPruneRp pimRPMappingNotificationPeriod pimRPMappingChangecount pimInterfaceElectionNotificationPeriod pimInterfaceElectionWinCount pimRefreshInterval pimInterfaceTable pimInterfaceEntry pimInterfaceIfIndex pimInterfaceIPVersion pimInterfaceAddressType pimInterfaceAddress pimInterfaceDR pimInterfaceHelloInterval pimInterfaceTrigHelloInterval pimInterfaceJoinPruneInterval pimInterfaceDFElectionRobustness pimInterfaceHelloHoldtime pimInterfaceJoinPruneHoldtime pimInterfacePropagationDelay pimInterfaceOverrideInterval pimInterfaceGenerationIDValue pimInterfaceDRPriority pimInterfacelanDelayEnabled pimInterfaceEffectPropagDelay pimInterfaceEffectOverrideIvl pimInterfaceSuppressionEnabled pimInterfaceBidirCapable pimInterfaceDRPriorityEnabled pimInterfaceDomainBorder pimInterfacestatus pimInterfacestubInterface 
pimInterfacePruneLimitInterval pimInterfaceSRPriorityEnabled pimNeighborTable pimNeighborEntry pimNeighborIfIndex pimNeighborAddress Type pimNeighborAddress pimNeighborUpTime pimNeighborExpiryTime pimNeighborLanPruneDelayPresent pimNeighborPropagationDelay pimNeighborOverrideInterval pimNeighborTBit pimNeighborGenerationIDPresent pimNeighborGenerationIDValue pimNeighborBidirCapable pimNeighborDRPriorityPresent pimNeighborDRPriority pimNeighborSRCapable pimNbrSecAddressTable pimNbrSecAddressEntry pimNbrSecAddressIfIndex pimNbrSecAddressType pimNbrSecAddressPrimary pimNorSecAddress pimStarGTable pimstarGEntry pimstarGAddressType pimStarGGrpAddress pimStarGUpTime pimStarGPimMode pimstarGRPAddressType pimStarGRPAddress pimStarGPimModeOrigin pimStarGRPIsLocal pimStarGUpstreamJoinState pimStarGUpstreamJoinTimer pimStarGUpstreamNeighborType pimStarGUpstreamNeighbor pimStarGRPFIfIndex pimStarGRPFNextHopType pimStarGRPFNextHop pimStarGRPFRouteProtocol pimStarGRPFRouteAddress pimStarGRPFRoutePrefixLength pimStarGRPFRouteMetricPref pimstarGRPFRouteMetric pimStarGITable pimStarGIEntry pimstarGIIfIndex pimStarGIUpTime pimStarGILocalMembership pimstarGIJoinPrunestate pimstarGIPrunePendingTimer pimStarGIJoinExpiryTimer pimstarGIAssertstate pimStarGIAssertTimer pimstarGIAssertWinnerAddressType pimStarGIAssertWinnerAddress pimstarGIAssertWinnerMetricPref pimStarGIAssertWinnerMetric pimSGTable pimSGEntry pimSGAddressType pimSGGrpAddress pimSGSrcAddress pimSGUPTime pimSGPimMode pimSGUpstreamJoinstate pimSGUpstreamJoinTimer pimSGUpstreamNeighbor pimSGRPFIfIndex pimSGRPFNextHopType pimSGRPFNextHop pimSGRPFRouteProtocol pimSGRPFRouteAddress pimSGRPFRoutePrefixLength pimSGRPFRouteMetricPref pimSGRPFRouteMetric pimSGSPTBit pimSGKeepaliveTimer pimSGDRRegisterState pimSGDRRegisterStopTimer pimSGRPRegisterPMBRAddressType pimSGRPRegisterPMBRAddress pimSGUpstreamPruneState pimSGUpstreamPruneLimitTimer pimSGOriginatorState pimSGSourceActiveTimer pimSGStateRefreshTimer pimSGITable pimSGIEntry pimSGIIfIndex pimSGIUpTime pimSGILocalMembership pimSGIJoinPrunestate pimSGIPrunePendingTimer pimSGIJoinExpiryTimer pimSGIAssertstate pimSGIAssertTimer pimSGIAssertWinnerAddressType pimSGIAssertWinnerAddress pimSGIAssertWinnerMetricPref pimSGIAssertWinnerMetric pimSGRptTable pimSGRptEntry pimSGRptSrcAddress pimSGRptUpTime pimSGRptUpstreamPruneState pimSGRptUpstreamoverrideTimer pimSGRptITable pimSGRptIEntry pimSGRptIIfIndex pimSGRptIUpTime pimSGRptILocalMembership pimSGRptIJoinPrunestate pimSGRptIPrunePendingTimer pimSGRptIPruneExpiryTimer pimBidirDFElectionTable pimBidirDFElectionEntry pimBidirDFElectionAddressType pimBidirDFElectionRPAddress pimBidirDFElectionIfIndex pimBidirDFElectionWinnerAddressType pimBidirDFElectionWinnerAddress pimBidirDFElectionWinnerUpTime 
pimBidirDFElectionWinnerMetricPref pimBidirDFElectionWinnerMetric pimBidirDFElectionstate pimBidirDFElectionstateTimer pimstaticRPTable pimstaticRPEntry pimstaticRPAddressType pimstaticRPGrpAddress pimstaticRPGrpPrefixLength pimstaticRPRPAddress pimstaticRPPimMode pimstaticRPOverrideDynamic pimStaticRPRowStatus pimstaticRPPrecedence pimAnycastRPSetTable pimAnycastRPSetEntry pimAnycastRPSetAddressType pimAnycastRPSetAnycastAddress pimAnycastRPSetRouterAddress pimAnycastRPSetRowStatus pimAnycastRPSetLocalRouter pimGroupMappingTable pimGroupMappingEntry pimGroupMappingorigin pimGroupMappingAddress Type pimGroupMappingGrpAddress pimGroupMappingGrpPrefixLength pimGroupMappingRPAddress pimGroupMappingPimMode pimGroupMappingPrecedence

There is also a specific danger arising from the notification pimInvalidRegister. This is originated by devices that receive an incorrect unicast-encapsulated multicast data packet, which poses a clear danger of propagating a Dos (Denial of Service) attack from the data or control plane to the network management plane. The following steps are taken to guard against this.

1. The notification is disabled by default. The writeable field pimInvalidRegisterNotificationPeriod must be set in order to enable it.

2. The syntax of pimInvalidRegisterNotificationPeriod prevents any given device from originating the notification more frequently than once every 10 seconds.

3. The counter pimInvalidRegisterMsgsRcvd provides equivalent function to the notification. Management applications are encouraged to monitor this counter in preference to enabling the notification.

The same measures are taken in respect of pimInvalidJoinPrune, though as this notification can only arise as a result of unroutable control packets, the risk is not so acute.

SNMP versions prior to SNMPv3 did not include adequate security. Even if the network itself is secure (for example by using IPsec), even then, there is no control as to who on the secure network is allowed to access and GET/SET (read/change/create/delete) the objects in this MIB module.

It is RECOMMENDED that implementers consider the security features as provided by the SNMPV3 framework (see [RFC3410], section 8), including full support for the SNMPv3 cryptographic mechanisms (for authentication and privacy). 
Further, deployment of SNMP versions prior to SNMPv3 is NOT RECOMMENDED. Instead, it is RECOMMENDED to deploy SNMPv3 and to enable cryptographic security. It is then a customer/operator responsibility to ensure that the SNMP entity giving access to an instance of this MIB module is properly configured to give access to the objects only to those principals (users) that have legitimate rights to indeed GET or SET (change/create/delete) them.

\section{IANA Considerations}

PIM-STD-MIB is rooted under the mib-2 subtree. IANA has assigned $\{$ mib-2 157 \} to the PIM-STD-MIB module specified in this document.

\section{Acknowledgements}

This MIB module is based on the original work in RFC 2934 [RFC2934] by K. McCloghrie, D. Farinacci, D. Thaler, and W. Fenner and has been updated based on feedback from the IETF's Protocol Independent Multicast (PIM) Working Group.

Jonathan Nicholas was the editor of early versions of this document, and contributed the objects for management of PIM-DM.

9. References

9.1. Normative References

[RFC2119] Bradner, S., "Key words for use in RFCs to Indicate Requirement Levels", BCP 14, RFC 2119, March 1997.

[RFC2578] MCCloghrie, K., Ed., Perkins, D., Ed., and J. Schoenwaelder, Ed., "Structure of Management Information Version 2 (SMIV2)", STD 58, RFC 2578, April 1999.

[RFC2579] MCCloghrie, K., Ed., Perkins, D., Ed., and J. Schoenwaelder, Ed., "Textual Conventions for SMIv2", STD 58, RFC 2579, April 1999.

[RFC2580] MCCloghrie, K., Perkins, D., and J. Schoenwaelder, "Conformance Statements for SMIv2", STD 58, RFC 2580, April 1999.

[RFC2863] MCCloghrie, K. and F. Kastenholz, "The Interfaces Group MIB", RFC 2863, June 2000. 
[RFC3973] Adams, A., Nicholas, J., and W. Siadak, "Protocol Independent Multicast - Dense Mode (PIM-DM) : Protocol Specification (Revised)", RFC 3973, January 2005.

[RFC4001] Daniele, M., Haberman, B., Routhier, S., and J. Schoenwaelder, "Textual Conventions for Internet Network Addresses", RFC 4001, February 2005.

[RFC4601] Fenner, B., Handley, M., Holbrook, H., and I. Kouvelas, "Protocol Independent Multicast - Sparse Mode (PIM-SM) : Protocol Specification (Revised)", RFC 4601, August 2006.

[RFC4610] Farinacci, D. and Y. Cai, "Anycast-RP Using Protocol Independent Multicast (PIM)", RFC 4610, August 2006.

[RFC5015] Handley, M., Kouvelas, I., Speakman, T., and L. Vicisano, "Bidirectional Protocol Independent Multicast (BIDIR-PIM)", RFC 5015, October 2007.

[RFC5059] Bhaskar, N., Gall, A., Lingard, L., and S. Venaas, "Bootstrap Router (BSR) Mechanism for PIM", RFC 5059, January 2008 .

[RTPROTO] IANA, "IP Route Protocol MIB", September 2000, <http:/ /www.iana.org/assignments/ianaiprouteprotocol-mib>.

\subsection{Informative References}

[IPMCAST-MIB] MCWalter, D., "IP Multicast MIB", Work in Progress, August 2007 .

[RFC2932] McCloghrie, K., Farinacci, D., and D. Thaler, "IPv4 Multicast Routing MIB", RFC 2932, October 2000.

[RFC2934] MCCloghrie, K., Farinacci, D., Thaler, D., and B. Fenner, "Protocol Independent Multicast MIB for IPv4", RFC 2934, October 2000.

[RFC3376] Cain, B., Deering, S., Kouvelas, I., Fenner, B., and A. Thyagarajan, "Internet Group Management Protocol, Version 3", RFC 3376, October 2002.

[RFC3410] Case, J., Mundy, R., Partain, D., and B. Stewart, "Introduction and Applicability statements for Internet-Standard Management Framework", RFC 3410, December 2002 . 
[RFC3569]

$[\mathrm{RFC} 3618]$

$[\mathrm{RFC} 3810]$

[RFC3956]
Bhattacharyya, S., "An Overview of Source-Specific Multicast (SSM)", RFC 3569, July 2003.

Fenner, B. and D. Meyer, "Multicast Source Discovery Protocol (MSDP)", RFC 3618, October 2003.

Vida, R. and L. Costa, "Multicast Listener Discovery Version 2 (MLDv2) for IPv6", RFC 3810, June 2004.

Savola, P. and B. Haberman, "Embedding the Rendezvous Point (RP) Address in an IPv6 Multicast Address", RFC 3956, November 2004. 


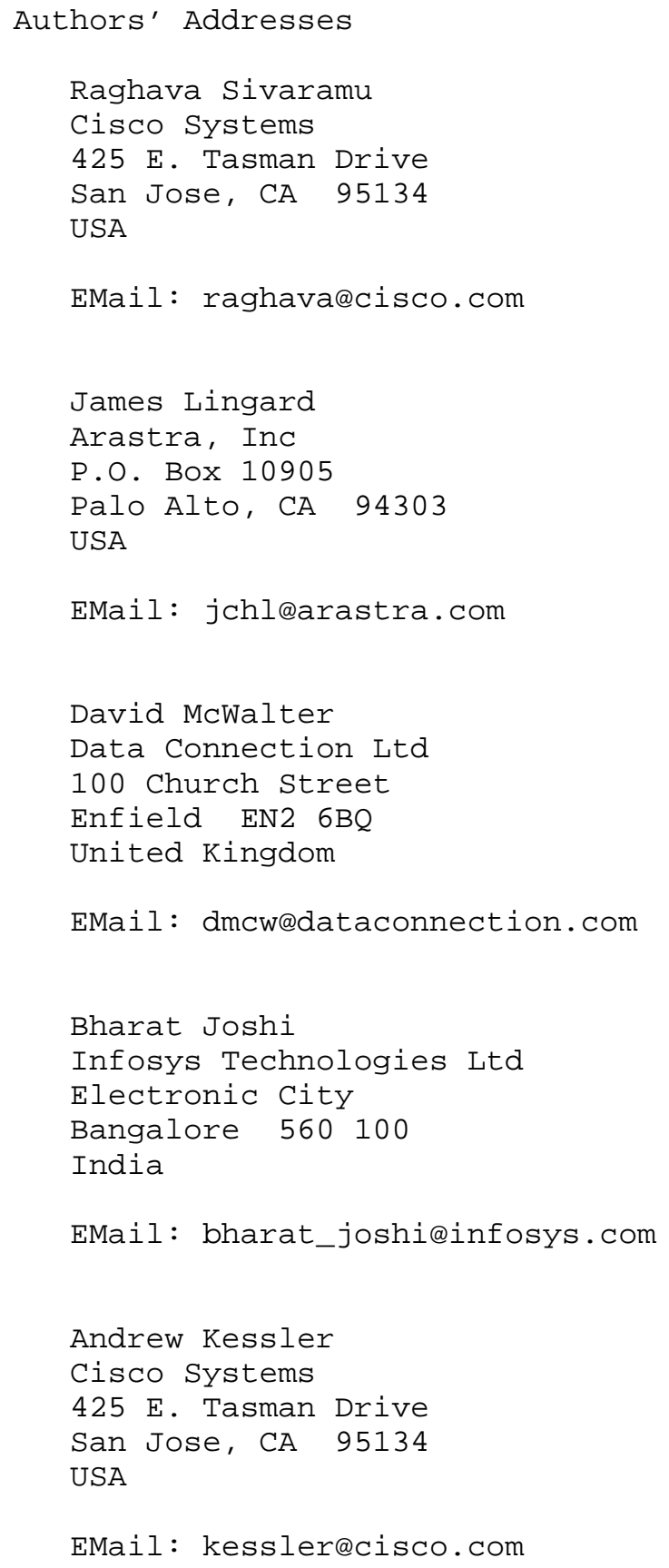

EMail: kesslerdcisco.com 
Full Copyright statement

Copyright (C) The IETF Trust (2008).

This document is subject to the rights, licenses and restrictions contained in BCP 78, and except as set forth therein, the authors retain all their rights.

This document and the information contained herein are provided on an "AS IS" basis and THE CONTRIBUTOR, THE ORGANIZATION HE/SHE REPRESENTS OR IS SPONSORED BY (IF ANY), THE INTERNET SOCIETY, THE IETF TRUST AND THE INTERNET ENGINEERING TASK FORCE DISCLAIM ALL WARRANTIES, EXPRESS OR IMPLIED, INCLUDING BUT NOT LIMITED TO ANY WARRANTY THAT THE USE OF THE INFORMATION HEREIN WILL NOT INFRINGE ANY RIGHTS OR ANY IMPLIED WARRANTIES OF MERCHANTABILITY OR FITNESS FOR A PARTICULAR PURPOSE.

Intellectual Property

The IETF takes no position regarding the validity or scope of any Intellectual Property Rights or other rights that might be claimed to pertain to the implementation or use of the technology described in this document or the extent to which any license under such rights might or might not be available; nor does it represent that it has made any independent effort to identify any such rights. Information on the procedures with respect to rights in RFC documents can be found in BCP 78 and BCP 79 .

Copies of IPR disclosures made to the IETF Secretariat and any assurances of licenses to be made available, or the result of an attempt made to obtain a general license or permission for the use of such proprietary rights by implementers or users of this specification can be obtained from the IETF on-line IPR repository at http://www.ietf.org/ipr.

The IETF invites any interested party to bring to its attention any copyrights, patents or patent applications, or other proprietary rights that may cover technology that may be required to implement this standard. Please address the information to the IETF at ietf-ipreietf.org. 